
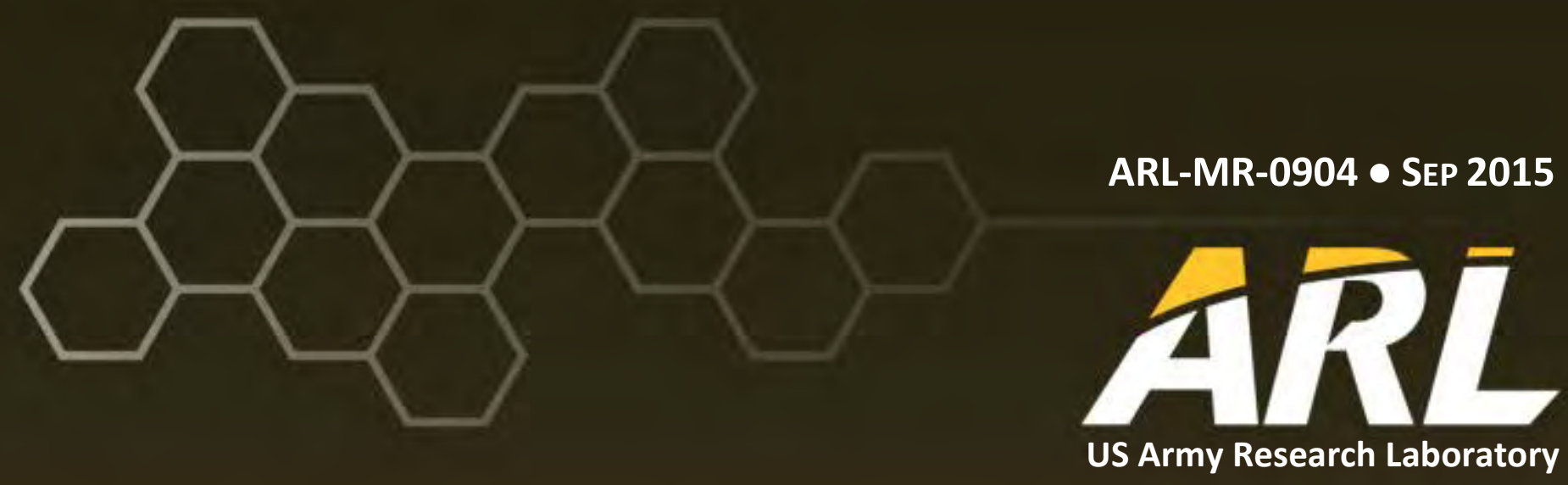

\title{
Ballistic Evaluation of 6055 Aluminum
}

\author{
by Denver B Gallardy
}




\section{NOTICES}

\section{Disclaimers}

The findings in this report are not to be construed as an official Department of the Army position unless so designated by other authorized documents.

Citation of manufacturer's or trade names does not constitute an official endorsement or approval of the use thereof.

Destroy this report when it is no longer needed. Do not return it to the originator. 
ARL-MR-0904 • SEP 2015

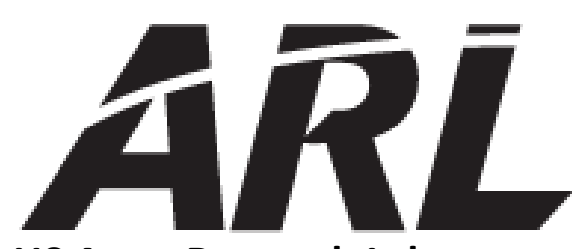

US Army Research Laboratory

\section{Ballistic Evaluation of 6055 Aluminum}

\section{by Denver B Gallardy}

Weapons and Materials Research Directorate, ARL 


\section{REPORT DOCUMENTATION PAGE}

Public reporting burden for this collection of information is estimated to average 1 hour per response, including the time for reviewing instructions, searching existing data sources, gathering and maintaining the data needed, and completing and reviewing the collection information. Send comments regarding this burden estimate or any other aspect of this collection of information, including suggestions for reducing the burden, to Department of Defense, Washington Headquarters Services, Directorate for Information Operations and Reports (0704-0188), 1215 Jefferson Davis Highway, Suite 1204, Arlington, VA $22202-4302$. Respondents should be aware that notwithstanding any other provision of law, no person shall be subject to any penalty for failing to comply with a collection of information if it does not display a currently valid OMB control number.

PLEASE DO NOT RETURN YOUR FORM TO THE ABOVE ADDRESS.

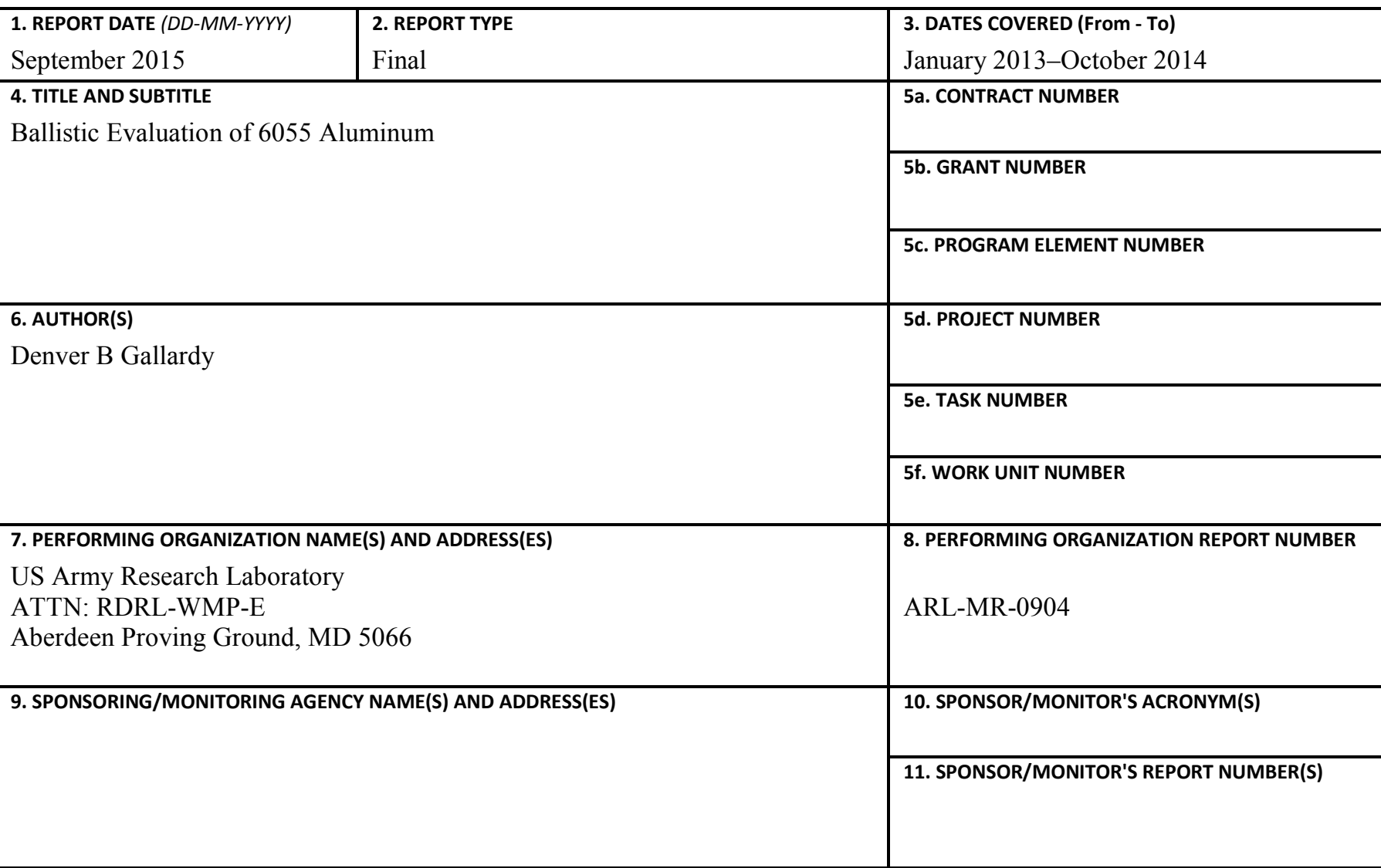

12. DISTRIBUTION/AVAILABILITY STATEMENT

Approved for public release; distribution is unlimited.

\section{SUPPLEMENTARY NOTES}

\section{ABSTRACT}

The US Army Research Laboratory (ARL) evaluated the ballistic performance of aluminum alloy (AA) 6055-T651 produced by Alcoa as part of a Defense Acquisition Challenge Program. Ballistic evaluation was performed using armor-piercing and fragment-simulating projectiles to determine the $\mathrm{V}_{50}$ ballistic-protection limit $\left(\mathrm{V}_{50}\right)$ for various thicknesses of material. The $\mathrm{V}_{50}$ was then compared to other ballistic-grade aluminum alloys, namely AA6061 and AA7039. The results of these experiments were used to derive the acceptance tables for AA6055 included in the updated military specification, MIL-DTL32262A (MR).

\section{SUBJECT TERMS}

aluminum, armor, V50, 6055, Defense Acquisition Challenge, ballistic acceptance

16. SECURITY CLASSIFICATION OF:
\begin{tabular}{l|l|l|} 
a. REPORT & b. ABSTRACT & c. THIS PAGE \\
Unclassified & Unclassified & Unclassified \\
\hline
\end{tabular}

\begin{tabular}{l|c|}
$\begin{array}{l}\text { 17. LIMITATION } \\
\text { OF ABSTRACT }\end{array}$ & $\begin{array}{l}\text { 18. NUMBER } \\
\text { OF PAGES }\end{array}$ \\
UU & 80 \\
\hline
\end{tabular}

19a. NAME OF RESPONSIBLE PERSON

Denver B Gallardy 19b. TELEPHONE NUMBER (Include area code) 410-278-6510 


\section{Contents}

List of Figures iv

List of Tables

Acknowledgments v v

$\begin{array}{ll}\text { 1. Introduction } & 1\end{array}$

2. Experimental Procedure 2

3. Test Projectiles 3

$\begin{array}{lll}3.1 & \text { Armor-Piercing (AP) Projectiles } & 3\end{array}$

3.2 Fragment-Simulating Projectiles (FSP) 4

4. Results and Analysis 5

5. Conclusions 11

6. References 12

$\begin{array}{ll}\text { Appendix A. Ballistic Test Data } & 13\end{array}$

Appendix B. Additional Ballistic Test Data 65

List of Symbols, Abbreviations, and Acronyms 69

$\begin{array}{ll}\text { Distribution List } & \mathbf{7 0}\end{array}$ 


\section{List of Figures}

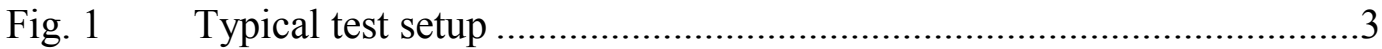

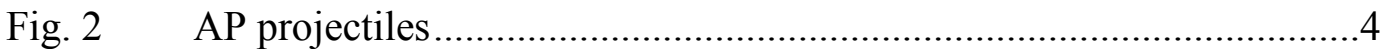

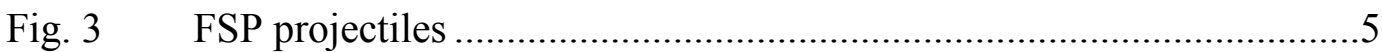

Fig. 4 Ballistic limit vs. thickness of AA6055 as compared to existing specs for the 0.30 -cal APM2 at $30^{\circ}$ obliquity ............................................

Fig. 5 Ballistic limit vs. thickness of AA6055 as compared to existing specs for the 0.30 -cal APM2 at $0^{\circ}$ obliquity .............................................. 9

Fig. 6 Ballistic limit vs. thickness of AA6055 as compared to existing specs

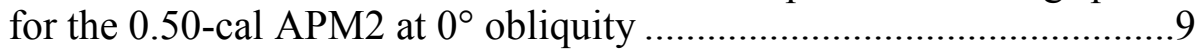

Fig. 7 Ballistic limit vs. thickness of AA6055 as compared to existing specs for the 0.50 -cal FSP at $0^{\circ}$ obliquity ............................................... 10

Fig. 8 Ballistic limit vs. thickness of AA6055 as compared to existing specs for the $20-\mathrm{mm}$ FSP at $0^{\circ}$ obliquity

\section{List of Tables}

Table 1 Test matrix for AA6055 indicating the number of plates tested............1

Table 2 Chemistry of AAs, weight-percent ranges ........................................2

Table 3 AP projectiles' physical characteristics ............................................4

Table $4 \quad 0.30$ cal. APM2, $30^{\circ}$ obliquity $\mathrm{V}_{50}$ ballistic limits for AA6055 ............5

Table $5 \quad 0.30$ cal. APM2, $0^{\circ}$ obliquity $V_{50}$ ballistic limits for AA6055 .............6

Table $6 \quad 0.50$ cal. APM2, $0^{\circ}$ obliquity $V_{50}$ ballistic limits for AA6055 .............6

Table $7 \quad$ 14.5-mm BS41, $0^{\circ}$ obliquity $\mathrm{V}_{50}$ ballistic limits for AA6055 ...............7

Table $8 \quad 0.50$ cal. FSP, $0^{\circ}$ obliquity $\mathrm{V}_{50}$ ballistic limits for AA6055 .................7

Table $9 \quad 20$-mm FSP, $0^{\circ}$ obliquity $\mathrm{V}_{50}$ ballistic limits for AA6055 ...................7

Table 10 Constants of regression for the acceptance curves for AA6055 ..........11 


\section{Acknowledgments}

The author would like to thank Alcoa, and specifically Phil Smith and Rick Kane, for producing and providing, in-kind, the armor-plate test articles. The author thanks Donald Little of the Armor Mechanisms Branch (AMB) US Army Research Laboratory (ARL), Phil Davis of Dynamic Science, Inc., and David Churn of Bowhead Science and Technology, LLC, for performing the ballistic experiments in Experimental Facilities (EFs) 108 and 106; also, John Hogan of AMB-ARL, Hugh Walter of Bowhead Science and Technology, and David Handshoe and William Bryant of Dynamic Science for performing the ballistic experiments in EF 110.

The author also acknowledges the outstanding contributions of the following people who made this work possible: Program lead Brian Placzankis of the ARL Coatings, Corrosion and Engineered Polymers Branch and William "Randy" Everett and Rino Imperiale of the Army Research, Development and Engineering Command's Comparative Technology Office. The author also thanks Robert "Gun Bob" Thompson from the Office of Secretary of Defense's Comparative Technology Office, Office of the Deputy Assistant Secretary of Defense for Emerging Capability and Prototyping. 
INTENTIONALLY LEFT BLANK. 


\section{Introduction}

In 2012, a Defense Acquisition Challenge (DAC) Program proposal was submitted to the Office of the Secretary of Defense (OSD) to provide improved armor-plate materials for production and repair of existing or new aluminum-based monocoque armored-vehicle hulls such as those of M2 Bradley Infantry Fighting Vehicles. Also in 2012, the Aluminum Association assigned a new 6XXX-series alloy designation to Alcoa for aluminum alloy (AA) 6055, granting it full commercial availability as rolled plate from Davenport, Iowa. AA6055 remains under patent protection and is solely manufactured by Alcoa. AA6055 was confirmed as having greater mechanical properties than the AA6061 alloy and therefore, became the basis for a fiscal-year 2012, OSD-funded DAC program to fully validate and ultimately transition AA6055 for availability as a weldable armor plate in US acquisition. The ballistics goal of this program was to verify that AA6055-T651 met or exceeded the performance of AA6061-T651. ${ }^{1}$

Several thicknesses of 6055-T651 were provided to the US Army Research Laboratory (ARL) by Alcoa. Table 1 is a summary matrix of the tested thicknesses subjected to impacts from various munitions including armor-piercing (AP) and fragment-simulating projectiles (FSPs). Additionally, Table 2 provides the required chemistries for AA6055 as well as other common aluminum-armor alloys.

Table 1 Test matrix for AA6055 indicating the number of plates tested

\begin{tabular}{|c|c|c|c|c|c|c|}
\hline $\begin{array}{c}\text { Nominal } \\
\text { Plate } \\
\text { Gage } \\
(\mathrm{mm}) \\
\end{array}$ & $\begin{array}{c}\text { 0.30-cal } \\
\text { APM2 } \\
30^{\circ} \\
\text { Obliquity }\end{array}$ & $\begin{array}{c}\text { 0.30-cal } \\
\text { APM2 } \\
0^{\circ} \\
\text { Obliquity } \\
\end{array}$ & $\begin{array}{c}\text { 0.50-cal } \\
\text { APM2 } \\
0^{\circ} \\
\text { Obliquity } \\
\end{array}$ & $\begin{array}{c}\text { 0.50-cal } \\
\text { FSP } \\
0^{\circ} \\
\text { Obliquity } \\
\end{array}$ & $\begin{array}{c}\text { 20-mm } \\
\text { FSP } \\
0^{\circ} \\
\text { Obliquity } \\
\end{array}$ & $\begin{array}{c}\text { 14.5-mm } \\
\text { BS41 } \\
0^{\circ} \\
\text { Obliquity } \\
\end{array}$ \\
\hline 12.70 & 3 & & & & 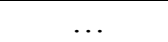 & $\ldots$ \\
\hline 19.05 & 2 & 2 & ... & 2 & $\ldots$ & $\ldots$ \\
\hline 25.40 & $\ldots$ & 3 & $\ldots$ & 3 & 3 & $\ldots$ \\
\hline 38.10 & $\ldots$ & 5 & 5 & $\ldots$ & 4 & $\ldots$ \\
\hline 50.80 & $\ldots$ & $\ldots$ & 5 & $\ldots$ & 5 & $\ldots$ \\
\hline 63.50 & $\ldots$ & $\ldots$ & 2 & $\ldots$ & $\ldots$ & $\ldots$ \\
\hline 76.20 & $\ldots$ & $\ldots$ & 1 & $\ldots$ & $\ldots$ & 1 \\
\hline
\end{tabular}


Table 2 Chemistry of AAs, weight-percent ranges ${ }^{2}$

\begin{tabular}{|c|c|c|c|c|c|c|c|c|}
\hline Element & 2139 & 2195 & 2519 & 5083 & 6061 & 6055 & 7039 & 7085 \\
\hline Copper & $4.5-5.5$ & $3.70-4.30$ & $5.30-6.40$ & $0.10 \max$ & $0.15-0.40$ & $0.50-1.0$ & $0.10 \max$ & $1.3-2.0$ \\
\hline Iron & $0.15 \max$ & $0.15 \max$ & $0.30 \max ^{\mathrm{a}}$ & $0.40 \max$ & $0.70 \max$ & $0.30 \max$ & $0.40 \max$ & $0.08 \max$ \\
\hline Lithium & $\ldots$ & $0.80-1.20$ & $\ldots$ & $\ldots$ & & & & $\cdots$ \\
\hline Chromium & $0.05 \max$ & $\ldots$ & $\ldots$ & $0.05-0.25$ & $0.04-0.35$ & $0.20-0.30$ & $0.15-0.25$ & $0.04 \max$ \\
\hline Manganese & $0.20-0.60$ & $0.25 \max$ & $0.10-0.50$ & $0.40-1.0$ & $0.15 \max$ & $0.10 \max$ & $0.10-0.40$ & $0.04 \max$ \\
\hline Magnesium & $0.20-0.80$ & $0.25-0.80$ & $0.05-0.40$ & $4.0-4.90$ & $0.8-1.2$ & $0.70-1.1$ & $2.30-3.30$ & $1.2-1.8$ \\
\hline Silicon & $0.10 \max$ & $0.12 \max$ & $0.25 \max ^{\mathrm{a}}$ & $0.40 \max$ & $0.40-0.80$ & $0.60-1.2$ & $0.30 \max$ & $0.06 \max$ \\
\hline Titanium & $0.15 \max$ & $0.10 \max$ & $0.02-0.10$ & $0.15 \max$ & $0.15 \max$ & $0.10 \max$ & $0.10 \max$ & $0.06 \max$ \\
\hline Zinc & $0.25 \max$ & $0.25 \max$ & $0.10 \max$ & $0.25 \max$ & $0.25 \max$ & $0.55-0.90$ & $3.50-4.50$ & $7.0-8.0$ \\
\hline Zirconium & & $0.08-0.16$ & $0.10-0.25$ & $\ldots$ & $\ldots$ & $\ldots$ & $\ldots$ & $0.08-0.15$ \\
\hline Silver & $0.15-0.60$ & $0.25-0.60$ & $\ldots$ & $\ldots$ & $\ldots$ & $\ldots$ & $\ldots$ & $\ldots$ \\
\hline $\begin{array}{l}\text { Others } \\
\text { (each) }\end{array}$ & $0.05 \max$ & $0.05 \max$ & $0.05 \max$ & $0.05 \max$ & $0.05 \max$ & $0.05 \max$ & $0.05 \max$ & $0.05 \max$ \\
\hline $\begin{array}{l}\text { Others } \\
\text { (total) }\end{array}$ & $0.15 \max$ & $0.15 \max$ & $0.15 \max$ & $0.15 \max$ & $0.15 \max$ & $0.15 \max$ & $0.15 \max$ & $0.15 \max$ \\
\hline Aluminum & Remainder & Remainder & Remainder & Remainder & Remainder & Remainder & Remainder & Remainder \\
\hline
\end{tabular}

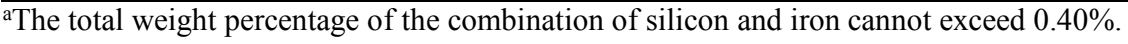

\section{Experimental Procedure}

The $\mathrm{V}_{50}$ is defined as the impact velocity at which the projectile is equally as likely to penetrate the target as it is to arrest. A $0.51-\mathrm{mm}(0.020$-inch) $2024 \mathrm{~T} 3$ aluminum witness plate is positioned $152 \mathrm{~mm}$ (6 inches) behind the target to determine the outcome of each shot. An impact is regarded as a complete penetration (CP), or loss, if the projectile or a resulting target fragment from impact creates a hole in the witness plate through which light can be observed. If an impact does not result in a $\mathrm{CP}$, it is considered a partial penetration (PP), or win. In order to keep results as consistent as possible, only shots conforming to the following conditions were used to determine the $\mathrm{V}_{50}$ : The projectile must be unyawed; $<2^{\circ}$ of total yaw for AP rounds and $<5^{\circ}$ of total yaw for FSPs; and strike the target at least 2 projectile diameters from any previous impact or damage or the edge of the target. Total yaw is defined as the vector sum of the projectile's pitch and yaw. The $V_{50}$ is calculated by the arithmetic mean of an equal number of CPs and PPs within an $18 \mathrm{~m} / \mathrm{s}(60$ $\mathrm{ft} / \mathrm{s})$ spread for a $2+2 \mathrm{~V}_{50}$; a $27 \mathrm{~m} / \mathrm{s}(90 \mathrm{ft} / \mathrm{s})$ spread for a $3+3 \mathrm{~V}_{50}$; and as small of a spread as attainable for a $5+5 \mathrm{~V}_{50}{ }^{3}$ 
Projectile velocities for the determination of the $V_{50}$ were measured using one of 2 methods as shown in Fig. 1. The first method is an orthogonal flash X-ray system as described in detail by Grabarek and Herr, ${ }^{4}$ which also measures pitch and yaw. The second method uses 3 infrared (IR) screens and a chronograph. The velocity is calculated using the first and third screens with the middle screen used to check for bad readings. The flash X-ray method was used in situations with projectiles that historically exhibit excessive yaw or if space did not allow for the use of the IR break screens. When the IR break screens and chronograph were used, the projectile velocity was corrected to the target-impact location using a correction factor based on an initial flash X-ray reading at the impact location. The correction was made using Eqs. 1 and 2 in lieu of utilizing air-drag factors:

$$
\frac{(\text { x-ray velocity })}{(\text { chronograph velocity })}=(\text { correction factor })
$$

$($ correction factor $) \times($ chronograph velocity $)=($ corrected velocity $)$.

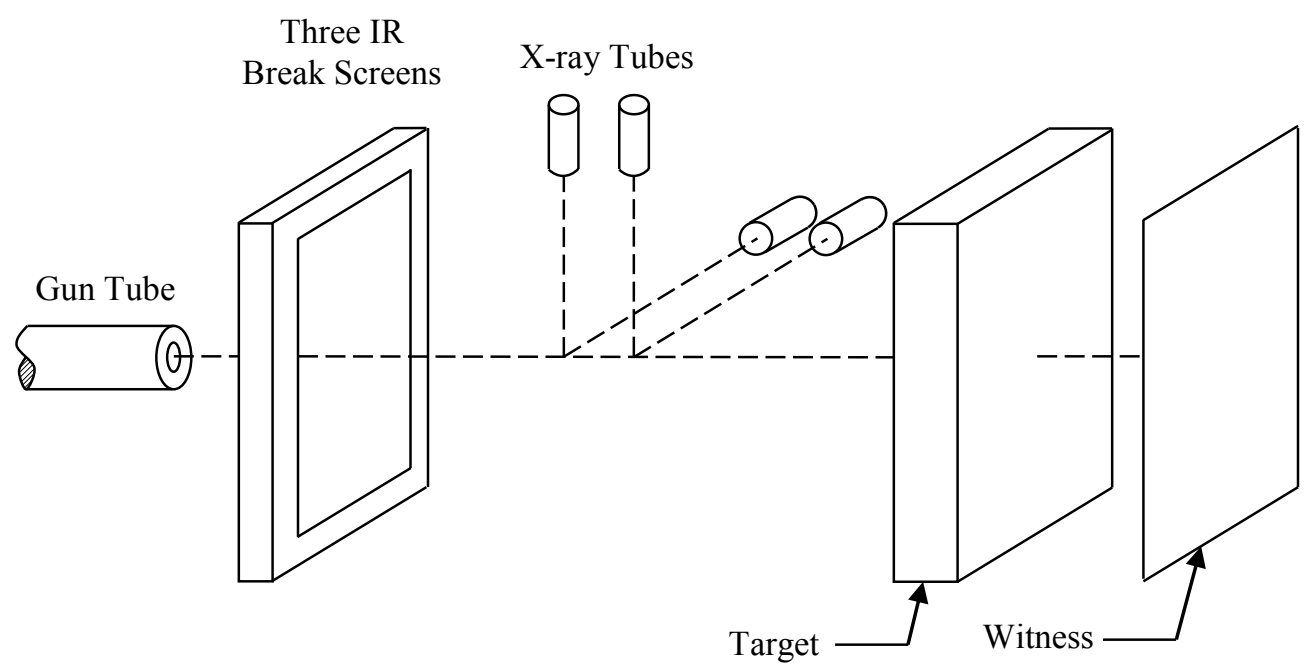

Fig. 1 Typical test setup

\section{Test Projectiles}

\subsection{Armor-Piercing (AP) Projectiles}

The US 0.30-cal. APM2 and 0.50-cal. APM2 and the Soviet 14.5-mm BS41 are the 3 AP projectiles that were used in this study. These projectiles are shown in Fig. 2. The APM2 projectiles have hardened steel cores with hardness of Rockwell C6163 whereas the BS41 has a tungsten carbide core. The physical characteristic of these projectiles are listed in Table 3. Additionally, a few experiments were 
repeated with the 0.30 -cal. APM2 Test Parts Kit (0.30-cal kit) round. This round is a US Army-authorized replacement for the historical 0.30-cal. APM2 due to the near depletion of the 0.30-cal. APM2 supply.

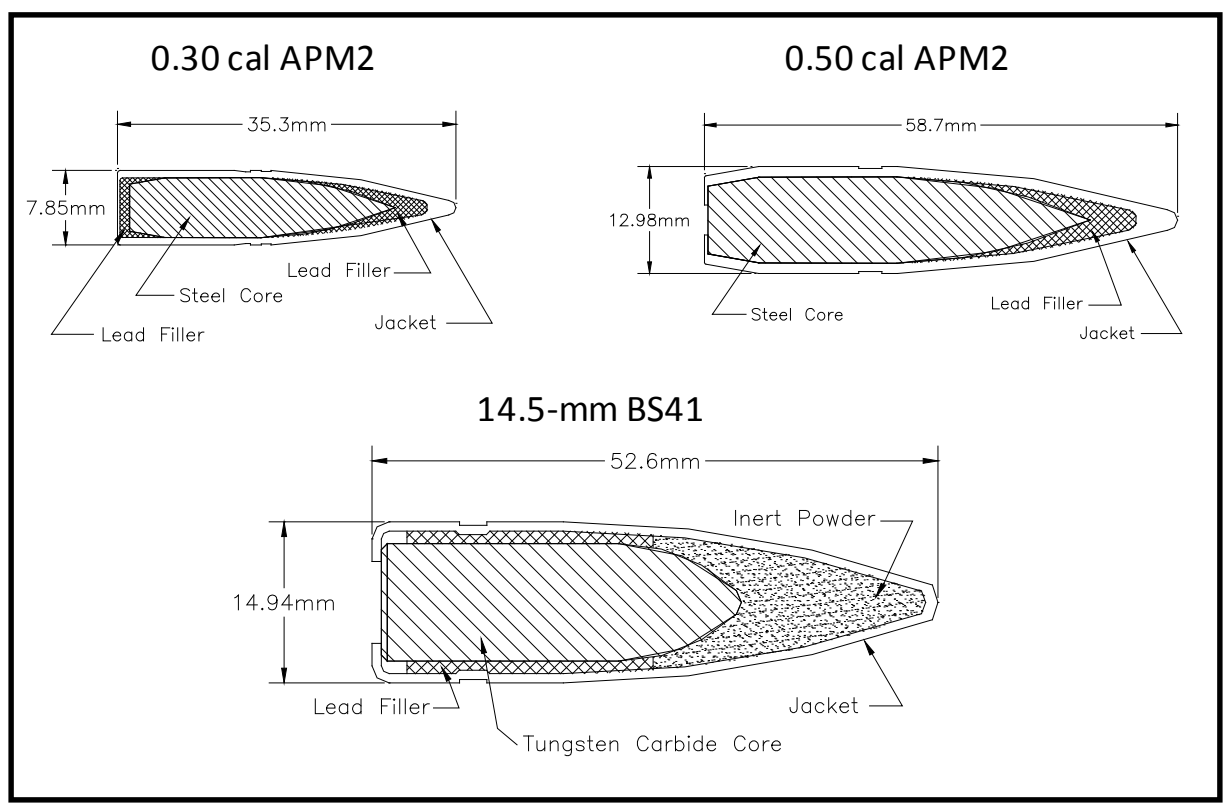

Fig. 2 AP projectiles

Table 3 AP projectiles' physical characteristics ${ }^{5}$

\begin{tabular}{|l|c|c|c|c|c|c|}
\hline \multirow{2}{*}{ Projectile Type } & \multicolumn{3}{|c|}{ Projectile } & \multicolumn{3}{c|}{ Core } \\
\cline { 2 - 7 } & $\begin{array}{c}\text { Length } \\
\text { (mm) }\end{array}$ & $\begin{array}{c}\text { Diameter } \\
(\mathbf{m m})\end{array}$ & $\begin{array}{c}\text { Weight } \\
\text { (g) }\end{array}$ & $\begin{array}{c}\text { Length } \\
(\mathbf{m m})\end{array}$ & $\begin{array}{c}\text { Diameter } \\
(\mathbf{m m})\end{array}$ & $\begin{array}{c}\text { Weight } \\
\text { (g) }\end{array}$ \\
\hline 0.30 -cal. APM2 & 35.3 & 7.85 & 10.8 & 27.4 & 6.2 & 5.3 \\
\hline 0.50 -cal. APM2 & 58.7 & 12.98 & 45.9 & 47.5 & 10.9 & 25.9 \\
\hline 14.5 -mm BS41 & 52.6 & 14.94 & 63.2 & 32.3 & 10.9 & 37.9 \\
\hline
\end{tabular}

\subsection{Fragment-Simulating Projectiles (FSP)}

FSPs (Fig. 3) are a family of projectiles that are flat-nosed, right circular cylinders manufactured to MIL-DTL-46593B (MR). ${ }^{6}$ These projectiles are used in material evaluations and acceptance testing to simulate performance against fragments produced from improvised explosive devices and artillery. Both 0.50-cal. and 20-mm FSPs were used for the evaluation of AA6055. 


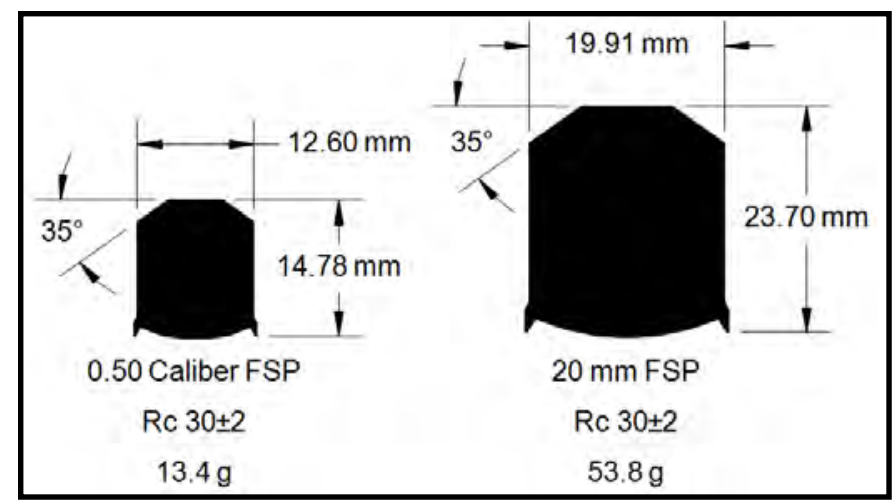

Fig. 3 FSP projectiles

\section{Results and Analysis}

The test results are summarized in Tables 4-9. The individual shot records are provided in Appendix A. Additionally, ballistic data obtained by the US Army, Aberdeen Test Center (ATC) ${ }^{7}$ are included in Appendix B.

Table 40.30 cal. APM2, 30 obliquity $V_{50}$ ballistic limits for AA6055

\begin{tabular}{|c|c|c|c|c|c|c|c|c|c|c|}
\hline \multirow[t]{2}{*}{ Plate ID } & \multicolumn{2}{|c|}{$\begin{array}{l}\text { Nominal } \\
\text { Thickness }\end{array}$} & \multicolumn{2}{|c|}{$\begin{array}{c}\text { Actual } \\
\text { Thickness }\end{array}$} & \multicolumn{2}{|c|}{ Areal Density } & \multicolumn{2}{|c|}{$V_{50}$} & \multicolumn{2}{|c|}{$\begin{array}{l}\text { Standard } \\
\text { Deviation }\end{array}$} \\
\hline & $(\mathrm{mm})$ & (in) & (mm) & (in) & $\left(\mathrm{kg} / \mathrm{m}^{2}\right)$ & $\left(\mathbf{l b} / \mathbf{f} \mathbf{t}^{2}\right)$ & $(\mathrm{m} / \mathrm{s})$ & $\mathrm{ft} / \mathrm{s}$ & $(\mathrm{m} / \mathrm{s})$ & $(\mathrm{ft} / \mathrm{s})$ \\
\hline $521-782$ & 12.70 & 0.50 & 12.93 & 0.509 & 35.07 & 7.18 & 482 & 1582 & 8 & 26 \\
\hline $521-782^{\mathrm{a}}$ & 12.70 & 0.50 & 12.93 & 0.509 & 35.07 & 7.18 & 479 & 1571 & 10 & 34 \\
\hline $521-792$ & 12.70 & 0.50 & 12.95 & 0.510 & 35.13 & 7.20 & 479 & 1573 & 7 & 24 \\
\hline $521-792$ & 12.70 & 0.50 & 12.98 & 0.511 & 35.20 & 7.21 & 474 & 1556 & 9 & 29 \\
\hline $521-802$ & 19.05 & 0.75 & 19.02 & 0.749 & 51.60 & 10.57 & 610 & 2001 & 10 & 32 \\
\hline $521-812$ & 19.05 & 0.75 & 19.08 & 0.751 & 51.74 & 10.60 & 609 & 1998 & 10 & 33 \\
\hline $521-812^{\mathrm{a}}$ & 19.05 & 0.75 & 19.08 & 0.751 & 51.74 & 10.60 & 614 & 2016 & 8 & 25 \\
\hline
\end{tabular}


Table 50.30 cal. APM2, $0^{\circ}$ obliquity $V_{50}$ ballistic limits for AA6055

\begin{tabular}{|c|c|c|c|c|c|c|c|c|c|c|}
\hline \multirow[t]{2}{*}{ Plate ID } & \multicolumn{2}{|c|}{$\begin{array}{l}\text { Nominal } \\
\text { Thickness }\end{array}$} & \multicolumn{2}{|c|}{ Actual Thickness } & \multicolumn{2}{|c|}{ Areal Density } & \multicolumn{2}{|c|}{$\mathbf{V}_{50}$} & \multicolumn{2}{|c|}{$\begin{array}{l}\text { Standard } \\
\text { Deviation }\end{array}$} \\
\hline & mm & in & mm & in & $\mathrm{kg} / \mathrm{m}^{2}$ & $\mathbf{l b} / \mathbf{f t}^{2}$ & $\mathbf{m} / \mathbf{s}$ & $\mathrm{ft} / \mathrm{s}$ & $\mathbf{m} / \mathbf{s}$ & $\mathbf{f t} / \mathrm{s}$ \\
\hline $521-802$ & 19.05 & 0.75 & 19.02 & 0.749 & 51.60 & 10.57 & 539 & 1770 & 11 & 37 \\
\hline $521-812$ & 19.05 & 0.75 & 19.08 & 0.751 & 51.74 & 10.60 & 537 & 1762 & 6 & 20 \\
\hline $521-812^{a}$ & 19.05 & 0.75 & 19.08 & 0.751 & 51.74 & 10.60 & 531 & 1743 & 6 & 21 \\
\hline $521-822$ & 25.40 & 1.00 & 25.25 & 0.994 & 68.48 & 14.03 & 618 & 2028 & 10 & 34 \\
\hline $521-822^{a}$ & 25.40 & 1.00 & 25.25 & 0.994 & 68.48 & 14.03 & 620 & 2033 & 6 & 21 \\
\hline $521-832$ & 25.40 & 1.00 & 25.68 & 1.011 & 69.65 & 14.27 & 634 & 2079 & 9 & 31 \\
\hline $521-842$ & 25.40 & 1.00 & 25.37 & 0.999 & 68.82 & 14.10 & 630 & 2067 & 8 & 25 \\
\hline $521-871$ & 38.10 & 1.50 & 37.97 & 1.495 & 102.99 & 21.09 & 798 & 2618 & 5 & 17 \\
\hline $521-871^{\mathrm{a}}$ & 38.10 & 1.50 & 37.97 & 1.495 & 102.99 & 21.09 & 800 & 2626 & 9 & 28 \\
\hline $521-872$ & 38.10 & 1.50 & 37.97 & 1.495 & 102.99 & 21.09 & 788 & 2585 & 8 & 26 \\
\hline $521-881$ & 38.10 & 1.50 & 38.02 & 1.497 & 103.13 & 21.12 & 803 & 2634 & 9 & 28 \\
\hline $521-882$ & 38.10 & 1.50 & 38.10 & 1.500 & 103.34 & 21.17 & 809 & 2654 & 8 & 25 \\
\hline $521-892$ & 38.10 & 1.50 & 38.05 & 1.498 & 103.20 & 21.14 & 808 & 2651 & 8 & 27 \\
\hline
\end{tabular}

a 0.30 -cal kit

Table 60.50 cal. APM2, $0^{\circ}$ obliquity $V_{50}$ ballistic limits for AA6055

\begin{tabular}{|c|c|c|c|c|c|c|c|c|c|c|}
\hline \multirow[t]{2}{*}{ Plate ID } & \multicolumn{2}{|c|}{$\begin{array}{l}\text { Nominal } \\
\text { Thickness }\end{array}$} & \multicolumn{2}{|c|}{$\begin{array}{c}\text { Actual } \\
\text { Thickness }\end{array}$} & \multicolumn{2}{|c|}{ Areal Density } & \multicolumn{2}{|c|}{$\mathrm{V}_{50}$} & \multicolumn{2}{|c|}{$\begin{array}{l}\text { Standard } \\
\text { Deviation }\end{array}$} \\
\hline & $(\mathbf{m m})$ & (in) & $(\mathrm{mm})$ & (in) & $\left(\mathrm{kg} / \mathrm{m}^{2}\right)$ & $\left(\mathbf{l b} / \mathbf{f t}^{2}\right)$ & $(\mathrm{m} / \mathrm{s})$ & $(\mathrm{ft} / \mathrm{s})$ & $(\mathrm{m} / \mathrm{s})$ & $\mathrm{ft} / \mathrm{s}$ \\
\hline $521-871$ & 38.10 & 1.50 & 37.92 & 1.493 & 102.85 & 21.07 & 585 & 1918 & 7 & 24 \\
\hline $521-872$ & 38.10 & 1.50 & 37.95 & 1.494 & 102.92 & 21.08 & 584 & 1915 & 7 & 24 \\
\hline $521-881$ & 38.10 & 1.50 & 38.02 & 1.497 & 103.13 & 21.12 & 584 & 1917 & 9 & 29 \\
\hline $521-882$ & 38.10 & 1.50 & 38.07 & 1.499 & 103.27 & 21.15 & 600 & 1969 & 9 & 28 \\
\hline $521-892$ & 38.10 & 1.50 & 38.05 & 1.498 & 103.20 & 21.14 & 603 & 1977 & 11 & 36 \\
\hline $521-901$ & 50.80 & 2.00 & 50.22 & 1.977 & 136.20 & 27.90 & 687 & 2254 & 5 & 15 \\
\hline $521-902$ & 50.80 & 2.00 & 45.47 & 1.790 & 123.31 & 25.26 & 686 & 2251 & 9 & 29 \\
\hline $521-911$ & 50.80 & 2.00 & 50.77 & 1.999 & 137.71 & 28.21 & 704 & 2309 & 5 & 16 \\
\hline $521-921$ & 50.80 & 2.00 & 50.90 & 2.004 & 138.06 & 28.28 & 697 & 2286 & 6 & 19 \\
\hline $521-922$ & 50.80 & 2.00 & 50.29 & 1.980 & 136.40 & 27.94 & 694 & 2278 & 6 & 21 \\
\hline $521-941$ & 63.50 & 2.50 & 63.50 & 2.500 & 172.23 & 35.28 & 797 & 2615 & 7 & 24 \\
\hline $521-942$ & 63.50 & 2.50 & 63.17 & 2.487 & 171.33 & 35.09 & 814 & 2671 & 8 & 27 \\
\hline $521-951$ & 76.20 & 3.00 & 74.12 & 2.918 & 201.02 & 41.17 & 906 & 2974 & 8 & 25 \\
\hline
\end{tabular}


Table $7 \quad$ 14.5-mm BS41, $0^{\circ}$ obliquity $V_{50}$ ballistic limits for AA6055

\begin{tabular}{|c|c|c|c|c|c|c|c|c|c|c|}
\hline \multirow[t]{2}{*}{ Plate ID } & \multicolumn{2}{|c|}{$\begin{array}{c}\text { Nominal } \\
\text { Thickness }\end{array}$} & \multicolumn{2}{|c|}{ Actual Thickness } & \multicolumn{2}{|c|}{ Areal Density } & \multicolumn{2}{|c|}{$V_{50}$} & \multicolumn{2}{|c|}{$\begin{array}{l}\text { Standard } \\
\text { Deviation }\end{array}$} \\
\hline & (mm) & (in) & (mm) & (in) & $\left(\mathrm{kg} / \mathrm{m}^{2}\right)$ & $\left(\mathbf{l b} / \mathbf{f t}^{2}\right)$ & $(\mathrm{m} / \mathrm{s})$ & $\mathbf{f t} / \mathbf{s})$ & $(\mathrm{m} / \mathrm{s})$ & $(\mathbf{f t} / \mathbf{s})$ \\
\hline $521-951$ & 76.20 & 3.00 & 74.12 & 2.918 & 201.02 & 41.17 & 839 & 2752 & 10 & 32 \\
\hline
\end{tabular}

Table 80.50 cal. FSP, $0^{\circ}$ obliquity $V_{50}$ ballistic limits for AA6055

\begin{tabular}{|c|c|c|c|c|c|c|c|c|c|c|}
\hline \multirow[t]{2}{*}{ Plate ID } & \multicolumn{2}{|c|}{$\begin{array}{l}\text { Nominal } \\
\text { Thickness }\end{array}$} & \multicolumn{2}{|c|}{ Actual Thickness } & \multicolumn{2}{|c|}{ Areal Density } & \multicolumn{2}{|c|}{$\mathbf{V}_{50}$} & \multicolumn{2}{|c|}{$\begin{array}{l}\text { Standard } \\
\text { Deviation }\end{array}$} \\
\hline & (mm) & (in) & (mm) & (in) & $\left(\mathrm{kg} / \mathrm{m}^{2}\right)$ & $\left(\mathbf{l b} / \mathbf{f t}^{2}\right)$ & $(\mathrm{m} / \mathrm{s})$ & $(\mathrm{ft} / \mathrm{s})$ & $(\mathrm{m} / \mathrm{s})$ & $(\mathrm{ft} / \mathrm{s})$ \\
\hline $521-802$ & 19.05 & 0.75 & 19.08 & 0.751 & 51.74 & 10.60 & 575 & 1888 & 4 & 13 \\
\hline $521-812$ & 19.05 & 0.75 & 19.13 & 0.753 & 51.87 & 10.62 & 588 & 1929 & 6 & 19 \\
\hline $521-822$ & 25.40 & 1.00 & 25.17 & 0.991 & 68.27 & 13.98 & 926 & 3037 & 9 & 28 \\
\hline $521-832$ & 25.40 & 1.00 & 25.65 & 1.010 & 69.58 & 14.25 & 949 & 3114 & 8 & 27 \\
\hline $521-842$ & 25.40 & 1.00 & 25.35 & 0.998 & 68.75 & 14.08 & 918 & 3012 & 9 & 28 \\
\hline
\end{tabular}

Table 9 20-mm FSP, $0^{\circ}$ obliquity $V_{50}$ ballistic limits for AA6055

\begin{tabular}{|c|c|c|c|c|c|c|c|c|c|c|}
\hline \multirow[t]{2}{*}{ Plate ID } & \multicolumn{2}{|c|}{$\begin{array}{c}\text { Nominal } \\
\text { Thickness }\end{array}$} & \multicolumn{2}{|c|}{$\begin{array}{c}\text { Actual } \\
\text { Thickness }\end{array}$} & \multicolumn{2}{|c|}{ Areal Density } & \multicolumn{2}{|c|}{$\mathrm{V}_{50}$} & \multicolumn{2}{|c|}{$\begin{array}{c}\text { Standard } \\
\text { Deviation }\end{array}$} \\
\hline & $(\mathrm{mm})$ & (in) & $(\mathrm{mm})$ & (in) & $(\mathrm{kg} / \mathrm{m}$ & $\left(\mathbf{l b} / \mathbf{f t}^{2}\right)$ & $(\mathrm{m} / \mathrm{s})$ & $(\mathbf{f t} / \mathbf{s})$ & $(\mathrm{m} / \mathrm{s})$ & $(\mathrm{ft} / \mathrm{s})$ \\
\hline $521-822$ & 25.40 & 1.00 & 25.17 & 0.991 & 68.27 & 13.98 & 442 & 1449 & 8 & 26 \\
\hline $521-832$ & 25.40 & 1.00 & 25.45 & 1.002 & 69.03 & 14.14 & 418 & 1372 & 5 & 16 \\
\hline $521-842$ & 25.40 & 1.00 & 25.40 & 1.000 & 68.89 & 14.11 & 422 & 1384 & 5 & 17 \\
\hline $521-871$ & 38.10 & 1.50 & 37.92 & 1.493 & 102.85 & 21.07 & 811 & 2661 & 8 & 26 \\
\hline $521-881$ & 38.10 & 1.50 & 38.02 & 1.497 & 103.13 & 21.12 & 817 & 2681 & 4 & 12 \\
\hline $521-$ & 38.10 & 1.50 & 37.92 & 1.493 & 102.85 & 21. & 782 & 2565 & 7 & 24 \\
\hline $521-8$ & 38.10 & 1.50 & 38.10 & 1.500 & 103 & 21. & 741 & 2432 & 8 & 25 \\
\hline $521-901$ & 50.80 & 2.00 & 50.72 & 1.997 & 137.57 & 28.18 & 1215 & 3985 & 9 & 30 \\
\hline $521-902$ & 50.80 & 2.00 & 50.39 & 1.984 & 136.68 & 27.99 & 1199 & 3935 & 9 & 31 \\
\hline $521-911$ & 50.80 & 2.00 & 50.77 & 1.999 & 137.71 & 28.21 & 1202 & 3943 & 7 & 24 \\
\hline $521-921$ & 50.80 & 2.00 & 50.90 & 2.004 & 138.06 & 28.28 & 1169 & 3836 & 5 & 16 \\
\hline $521-922$ & 50.80 & 2.00 & 50.55 & 1.990 & 137.09 & 28.08 & 1175 & 3854 & 5 & 17 \\
\hline
\end{tabular}

The results of the ballistic evaluation are compared directly against the acceptance curves of AA6061 in MIL-DTL-32262 and AA7039 in MIL-DTL-46063 ${ }^{8}$ for a higher-performing, 7XXX-series reference point. Figures 4-8 show the AA6055 test data collected by ARL and ATC as compared to the other specifications. The data displayed are the $V_{50}$ as a function of the plate thickness. To allow for a fair comparison against the existing specifications, a line depicting the $V_{50}-2 \sigma$ was plotted against the acceptance specs. This line represents a $V_{02}$ rather than a $V_{50}$. To ensure successful protection at a given thickness, the lower band of the $2 \sigma$ distribution $\left(\mathrm{V}_{02}\right.$ line) is used to define minimum-acceptable performance. A $\mathrm{V}_{50}$ falling below this line is considered unacceptable. For comparison purposes it should also be noted that the plates are compared on a thickness basis to be consistent with the specifications; however, the densities of the alloys vary slightly. 
Both AA6061 and AA6055 have a density of $2.71 \mathrm{~g} / \mathrm{cm}^{3}$ whereas AA7039 has a density of $2.74 \mathrm{~g} / \mathrm{cm}^{3}$.

As can be observed in the plots (Figs. 4-6), the AP performance for AA6055 is significantly higher than the existing AA6061 acceptance requirements. In fact, the data points approach the performance of AA7039 and in some cases actually exceed the AA7039 requirement. For the 0.30 -cal. APM2 at $30^{\circ}$ obliquity, the $V_{50}-2 \sigma$ line falls above the AA7039 spec for plate thicknesses less than 0.675 inches.

Turning to the FSP performance (Figs. 7 and 8), it can be observed that the performance of 6055 continues to behave more like AA7039 than AA6061. The 0.50-cal FSP performance yielded several data points above the AA7039 specification and the $\mathrm{V}_{50}-2 \sigma$ line fell slightly below the AA7039 specification. Similarly, for the 20-mm FSP, AA6055 exhibited a steeper performance curve like AA7039 as compared to the shallower AA6061 curve. Also, like AA7039 the 20-mm FSP performance drops below the AA6061 performance for thinner plates. This transition occurs at $32.64 \mathrm{~mm}$ (1.285 in) for AA6055, which is slightly higher than the $30.35 \mathrm{~mm}$ (1.195 in) transition of AA7039.

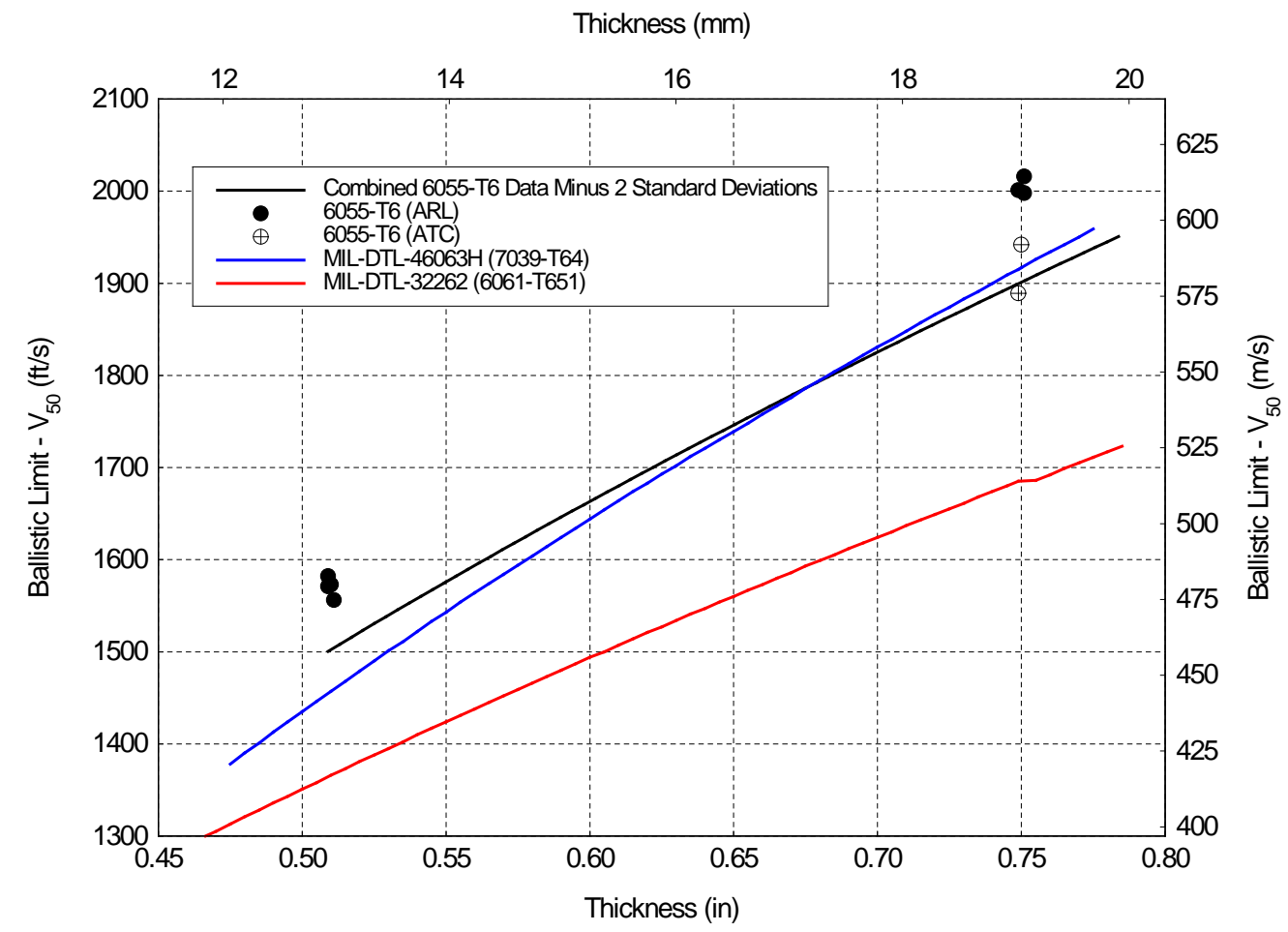

Fig. 4 Ballistic limit vs. thickness of AA6055 as compared to existing specs for the 0.30-cal APM2 at $30^{\circ}$ obliquity 
Thickness (mm)

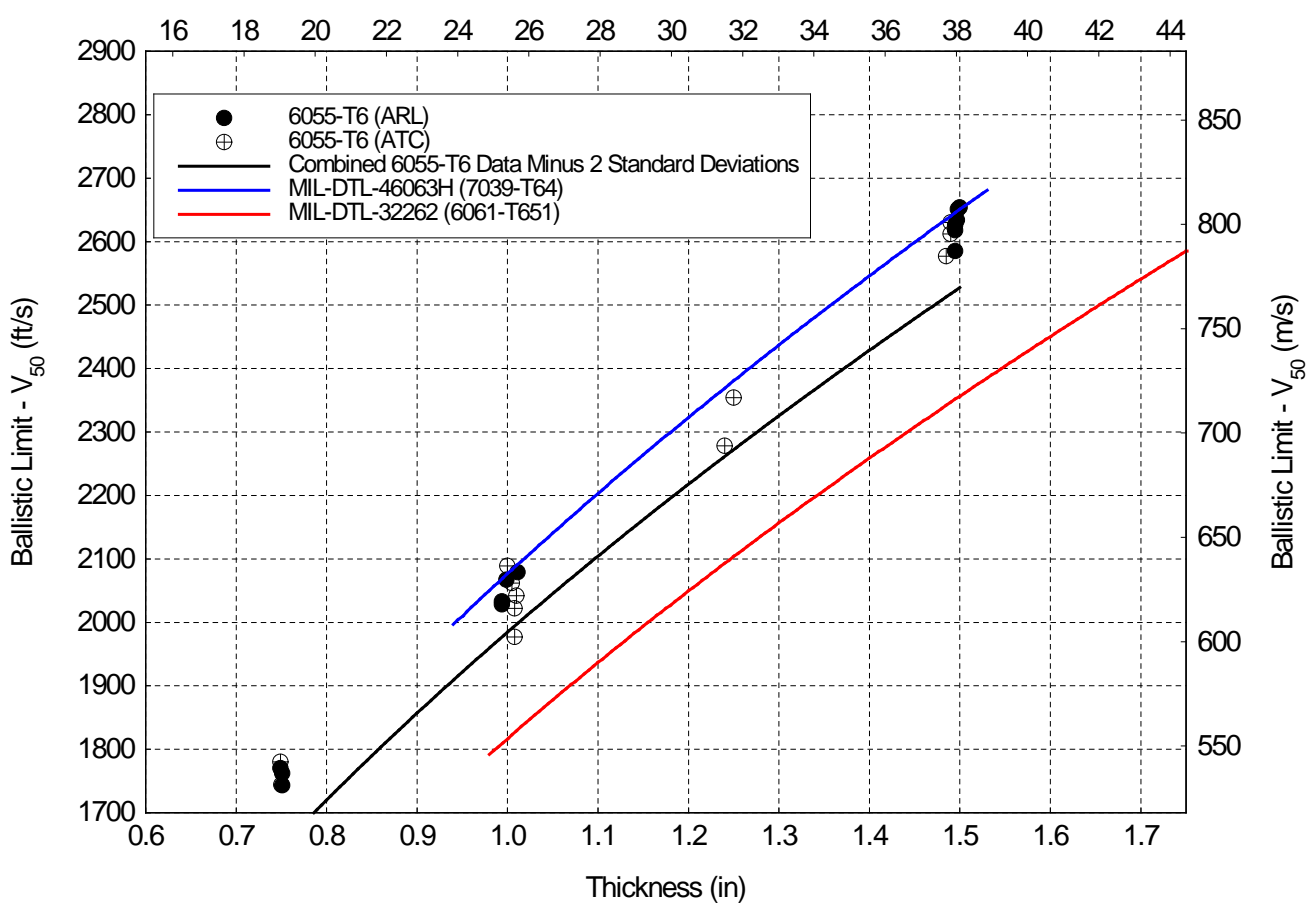

Fig. 5 Ballistic limit vs. thickness of AA6055 as compared to existing specs for the 0.30-cal APM2 at $0^{\circ}$ obliquity

Thickness (mm)

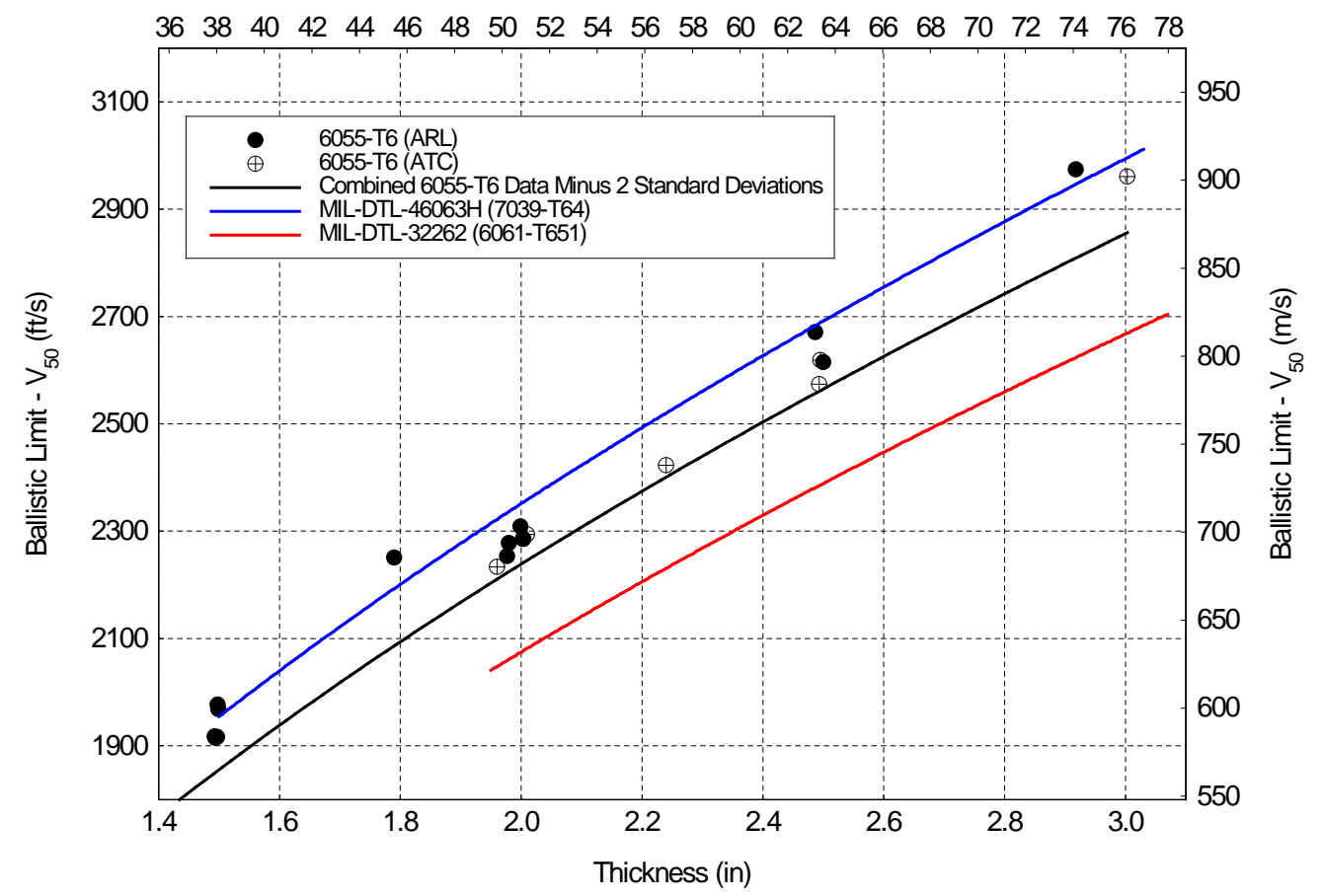

Fig. 6 Ballistic limit vs. thickness of AA6055 as compared to existing specs for the 0.50-cal APM2 at $0^{\circ}$ obliquity 


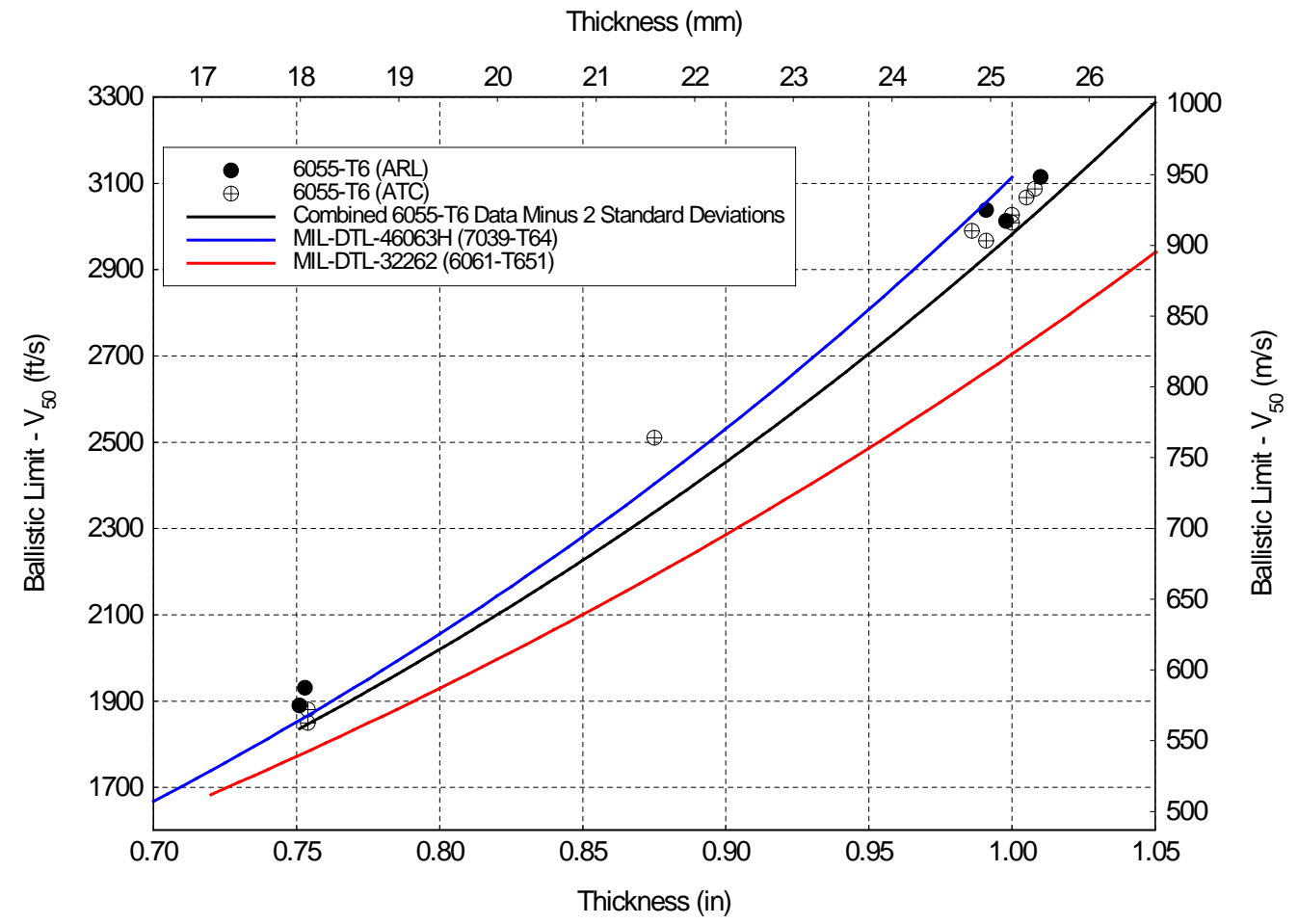

Fig. 7 Ballistic limit vs. thickness of AA6055 as compared to existing specs for the 0.50-cal FSP at $0^{\circ}$ obliquity

Thickness (mm)

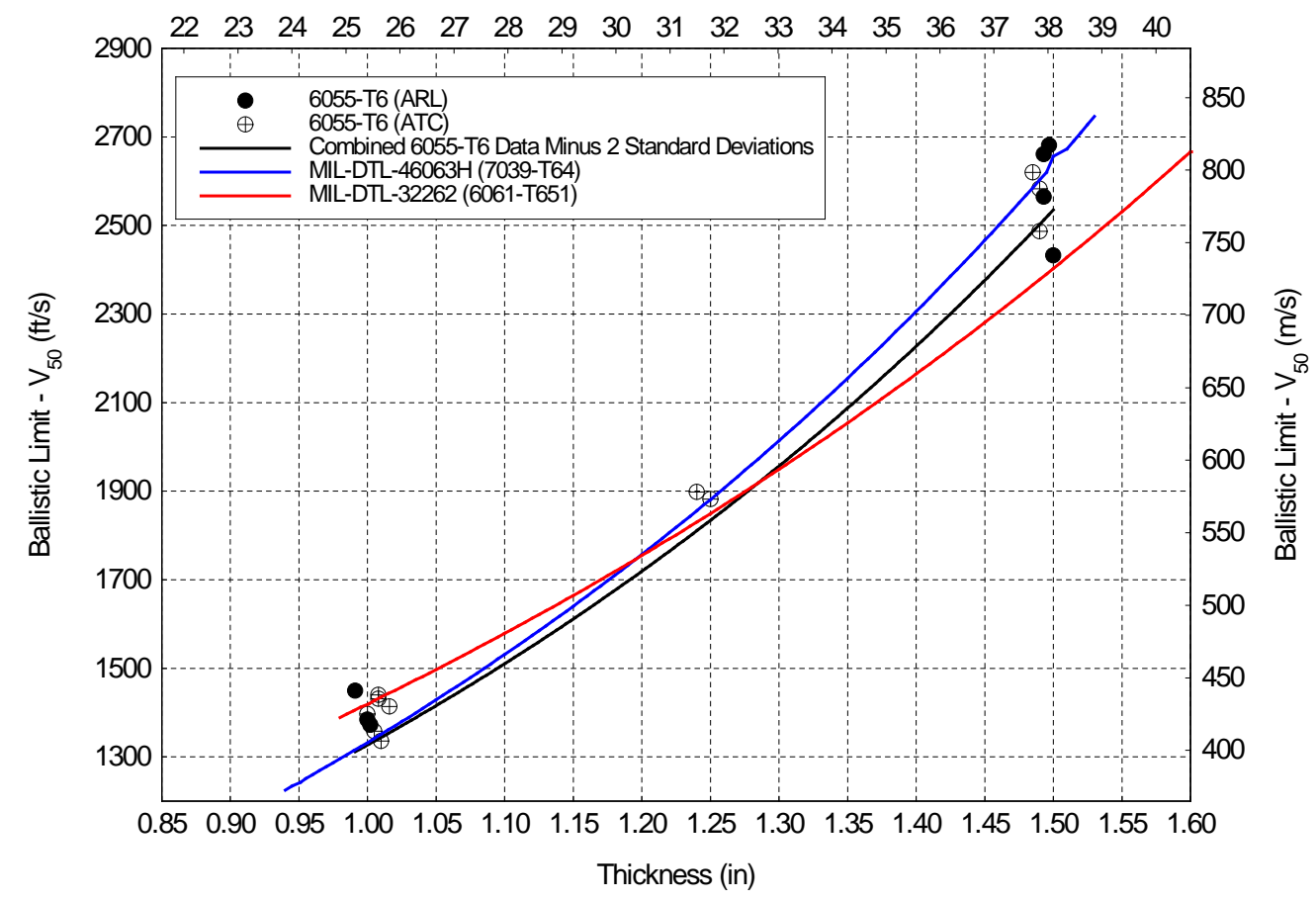

Fig. 8 Ballistic limit vs. thickness of AA6055 as compared to existing specs for the 20-mm FSP at $0^{\circ}$ obliquity 
The data collected by ARL, as well as data collected by ATC, ${ }^{7}$ were then used to generate acceptance tables for MIL-DTL-32262A (MR). The acceptance velocities were calculated by fitting the $\mathrm{V}_{50}$ data minus 2 standard deviations with Eqs. 3 and 4 for AP and FSP projectiles respectively. 9,10

$$
\begin{gathered}
V_{A}=1000 \sqrt{a+b t} \\
V_{A}=1000 e^{a+b t}
\end{gathered}
$$

In the above equations $V_{A}$ is the acceptance velocity, $t$ is the actual thickness of the plate, and both $a$ and $b$ are constants of regression. Table 10 lists the constants of regression and the Pearson's R correlation coefficient for each projectile. The ballistic tables corresponding to the acceptance curves can be found in MIL-DTL32262A (MR).

Table 10 Constants of regression for the acceptance curves for AA6055

\begin{tabular}{cccc}
\hline Projectile Type & \multicolumn{3}{c}{$\mathbf{6 0 5 5}$} \\
\hline & $\mathbf{a}$ & $\mathbf{b}$ & $\mathbf{R}$ \\
0.30 -cal AP, M2 at $30^{\circ}$ & -0.626 & 5.65 & 0.969 \\
0.30 -cal AP, M2 at $0^{\circ}$ & -0.961 & 4.90 & 0.988 \\
0.50 -cal AP, M2 at $0^{\circ}$ & -1.26 & 3.14 & 0.981 \\
0.50 -cal FSP at $0^{\circ}$ & -0.858 & 1.95 & 0.994 \\
20-mm FSP at $0^{\circ}$ & -1.01 & 1.29 & 0.987 \\
\hline
\end{tabular}

\section{Conclusions}

A ballistic evaluation has been performed on AA6055 in the T651 temper. This report has compared the performance of AA6055 against existing mil-spec, aluminum-armor material, namely AA6061 and AA7039. AA6055 performed better that AA6061 against both AP and FSP projectiles. The only exception is 20-mm FSP performance below 1.285 inches, where AA6061 exhibits higher performance. This report has also documented the calculations used to derive the acceptance tables included in the updated military specification, MIL-DTL32262A (MR). 


\section{References}

1. MIL-DTL-32262A (MR). Armor plate, aluminum alloy 6055 weldable \& alloy 6061, unweldable applique; Aberdeen Proving Ground (MD): Army Research Laboratory; 2015 Mar 16.

2. International alloy designations and chemical composition limits for wrought aluminum and wrought aluminum alloys. Arlington (VA): The Aluminum Association, Inc.; 2009 Feb.

3. MIL-STD-662F. V 50 ballistic test for armor. Aberdeen Proving Ground (MD): Army Research Laboratory; 1997 Dec 18.

4. Grabarek C, Herr L. X-ray multi-flash system for measurement of projectile performance at the target. Aberdeen Proving Ground (MD): Army Ballistic Research Laboratory (US): 1966 Sep. Report No.: BRL-TN-1634.

5. Mascianica F. Ballistic technology of lightweight armor. Watertown (MA): Army Materials Research Agency (US); 1964 Sep. Report No.: AMRA MS 64-07.

6. MIL-DTL-46593B (MR). Projectile, calibers .22, .30, .50, and $20 \mathrm{~mm}$ fragment-simulating. Aberdeen Proving Ground (MD): Army Research Laboratory; 2008 Aug 11.

7. Beavers K. Unpublished data on 6055. Aberdeen Proving Ground (MD): Army Aberdeen Test Center (US); 2013 Oct 9.

8. MIL-DTL-46063H. Armor plate, aluminum alloy, 7039. Aberdeen Proving Ground (MD): Army Research Laboratory; 1998 Sep 14.

9. DeLuca E, Anctil A. Laminate armor for light combat vehicles. Watertown (MA): Army Materials Technology Laboratory (US); 1986 Apr. Report No.: MTL TR 86-14.

10. Van Caneghem R, Typanski D, Latham R. Appendix C: Ballistic testing of aluminum armor alloys - shock testing of weldments and specification data. Aberdeen Proving Ground, MD: Army Combat Systems Test Activity (US); 1986 Apr. Report No.: MTL TR 86-14. 


\section{Appendix A. Ballistic Test Data}

This appendix appears in its original form, without editorial change. 


\section{$\underline{0.30-\text { cal APM2 }}$}

\begin{tabular}{|c|c|c|c|c|c|c|c|}
\hline \multicolumn{2}{|c|}{ Target: } & \multicolumn{4}{|c|}{ 6055-T651 } & Date: & $4 / 4 / 2013$ \\
\hline \multicolumn{2}{|c|}{ Plate Number: } & \multicolumn{4}{|c|}{$521-782$} & Location: & EF 106 \\
\hline \multicolumn{2}{|c|}{ Thickness, in: } & \multicolumn{4}{|l|}{0.509} & & \\
\hline \multicolumn{2}{|c|}{ Thickness, mm: } & \multicolumn{4}{|l|}{12.93} & & \\
\hline \multicolumn{2}{|c|}{ Hardness, BHN: } & \multicolumn{4}{|l|}{134} & & \\
\hline \multicolumn{2}{|c|}{ Obliquity: } & \multicolumn{4}{|l|}{$30^{\circ}$} & & \\
\hline \multicolumn{2}{|c|}{ Projectile: } & \multicolumn{4}{|c|}{0.30 cal APM2 } & & \\
\hline \multicolumn{2}{|c|}{$\begin{array}{l}\text { Velocity } \\
\text { Measurement: }\end{array}$} & \multicolumn{4}{|c|}{ Chrono } & & \\
\hline \multicolumn{2}{|c|}{$\mathrm{V}_{50}:$} & \multicolumn{2}{|c|}{$1582 \mathrm{ft} / \mathrm{s}$} & & \multicolumn{2}{|c|}{ Number of Shots: } & 4 \\
\hline \multirow{2}{*}{\multicolumn{2}{|c|}{$\begin{array}{r}\text { Std Dev: } \\
\text { ZMR: }\end{array}$}} & \multicolumn{2}{|l|}{$26 \mathrm{ft} / \mathrm{s}$} & & \multicolumn{2}{|r|}{ Spread: } & $58 \mathrm{ft} / \mathrm{s}$ \\
\hline & & \multicolumn{2}{|l|}{$16 \mathrm{ft} / \mathrm{s}$} & & & & \\
\hline \multicolumn{2}{|c|}{ Striking Velocity } & \multirow{2}{*}{$\begin{array}{l}\text { Pitch } \\
(\mathrm{deg})\end{array}$} & \multirow{2}{*}{$\begin{array}{l}\text { Yaw } \\
(\mathrm{deg})\end{array}$} & \multirow{2}{*}{$\begin{array}{l}\text { Result } \\
(\mathrm{PP} / \mathrm{CP})\end{array}$} & \multirow{2}{*}{$\begin{array}{c}\text { Used for } \\
\mathrm{V}_{50} \\
(\mathrm{Yes} / \mathrm{No})\end{array}$} & \multirow{2}{*}{$\begin{array}{c}\text { Shot } \\
\text { Number }\end{array}$} & \multirow[t]{2}{*}{ Comments } \\
\hline$(\mathrm{ft} / \mathrm{s})$ & $(\mathrm{m} / \mathrm{s})$ & & & & & & \\
\hline 1645 & 501 & -- & -- & $\mathrm{CP}$ & No & 12530 & \\
\hline 1566 & 477 & -- & -- & $\mathbf{C P}$ & Yes & 12531 & \\
\hline 1467 & 447 & -- & -- & PP & No & 12532 & \\
\hline 1468 & 447 & -- & -- & $\mathrm{PP}$ & No & 12533 & \\
\hline 1511 & 461 & -- & -- & PP & No & 12534 & \\
\hline 1530 & 466 & -- & -- & PP & No & 12535 & \\
\hline 1582 & 482 & -- & -- & PP & Yes & 12536 & \\
\hline 1619 & 493 & -- & -- & CP & Yes & 12537 & \\
\hline 1561 & 476 & -- & -- & PP & Yes & 12538 & \\
\hline & & & & & & & \\
\hline & & & & & & & \\
\hline & & & & & & & \\
\hline
\end{tabular}




\begin{tabular}{|c|c|c|c|c|c|c|c|}
\hline \multicolumn{2}{|l|}{ Target: } & \multicolumn{4}{|c|}{ 6055-T651 } & Date: & 4/9/2013 \\
\hline \multicolumn{2}{|c|}{ Plate Number: } & \multicolumn{4}{|c|}{$521-782$} & Location: & EF 106 \\
\hline \multicolumn{2}{|c|}{ Thickness, in: } & \multicolumn{4}{|c|}{0.509} & & \\
\hline \multicolumn{2}{|c|}{ Thickness, mm: } & \multicolumn{4}{|l|}{12.93} & & \\
\hline \multicolumn{2}{|c|}{ Hardness, BHN: } & \multicolumn{4}{|l|}{134} & & \\
\hline \multicolumn{2}{|c|}{ Obliquity: } & \multicolumn{4}{|l|}{$30^{\circ}$} & & \\
\hline \multicolumn{2}{|c|}{ Projectile: } & \multicolumn{4}{|c|}{0.30 cal Kit } & & \\
\hline \multicolumn{2}{|c|}{$\begin{array}{l}\text { Velocity } \\
\text { Measurement: }\end{array}$} & \multicolumn{4}{|c|}{ Chrono } & & \\
\hline & $\mathrm{V}_{50}$ & \multicolumn{2}{|c|}{$1571 \mathrm{ft} / \mathrm{s}$} & & \multicolumn{2}{|c|}{ Number of Shots: } & 6 \\
\hline & Std Dev & \multicolumn{2}{|c|}{$34 \mathrm{ft} / \mathrm{s}$} & & \multicolumn{2}{|r|}{ Spread: } & $82 \mathrm{ft} / \mathrm{s}$ \\
\hline & ZMR & \multicolumn{2}{|l|}{ N/A } & & & & \\
\hline \multicolumn{2}{|c|}{ Striking Velocity } & \multirow{2}{*}{$\begin{array}{l}\text { Pitch } \\
\text { (deg) }\end{array}$} & \multirow{2}{*}{$\begin{array}{l}\text { Yaw } \\
\text { (deg) }\end{array}$} & \multirow{2}{*}{$\begin{array}{l}\text { Result } \\
(\mathrm{PP} / \mathrm{CP})\end{array}$} & \multirow{2}{*}{$\begin{array}{c}\text { Used for } \\
V_{50} \\
(\mathrm{Yes} / \mathrm{No})\end{array}$} & \multirow{2}{*}{$\begin{array}{c}\text { Shot } \\
\text { Number }\end{array}$} & \multirow[t]{2}{*}{ Comments } \\
\hline$(\mathrm{ft} / \mathrm{s})$ & $(\mathrm{m} / \mathrm{s})$ & & & & & & \\
\hline 1652 & 504 & -- & -- & $\mathrm{CP}$ & No & 12539 & \\
\hline 1529 & 466 & -- & -- & PP & Yes & 12540 & \\
\hline 1586 & 483 & -- & -- & $\mathbf{C P}$ & Yes & 12541 & \\
\hline 1528 & 466 & -- & -- & $\mathbf{P P}$ & Yes & 12542 & \\
\hline 1524 & 464 & -- & -- & PP & No & 12543 & \\
\hline 1610 & 491 & -- & -- & CP & Yes & 12544 & \\
\hline 1579 & 481 & -- & -- & PP & Yes & 12545 & \\
\hline 1592 & 485 & -- & -- & $\mathbf{C P}$ & Yes & 12546 & \\
\hline & & & & & & & \\
\hline & & & & & & & \\
\hline & & & & & & & \\
\hline & & & & & & & \\
\hline
\end{tabular}




\begin{tabular}{|c|c|c|c|c|c|c|c|}
\hline \multirow{2}{*}{\multicolumn{2}{|c|}{$\begin{array}{l}\text { Target: } \\
\text { Plate Number: }\end{array}$}} & \multicolumn{4}{|c|}{ 6055-T651 } & Date: & $4 / 9 / 2013$ \\
\hline & & \multicolumn{4}{|c|}{$521-792$} & Location: & EF 106 \\
\hline \multicolumn{2}{|c|}{ Thickness, in: } & \multicolumn{4}{|c|}{0.510} & & \\
\hline \multicolumn{2}{|c|}{ Thickness, mm: } & \multicolumn{4}{|l|}{12.95} & & \\
\hline \multicolumn{2}{|c|}{ Hardness, BHN: } & \multicolumn{4}{|l|}{137} & & \\
\hline \multicolumn{2}{|c|}{ Obliquity: } & \multicolumn{4}{|l|}{$30^{\circ}$} & & \\
\hline \multicolumn{2}{|c|}{ Projectile: } & \multicolumn{4}{|c|}{0.30 cal APM2 } & & \\
\hline \multicolumn{2}{|c|}{$\begin{array}{l}\text { Velocity } \\
\text { Measurement: }\end{array}$} & \multicolumn{4}{|c|}{ Chrono } & & \\
\hline \multicolumn{2}{|c|}{$\mathrm{V}_{50}:$} & \multicolumn{2}{|c|}{$1573 \mathrm{ft} / \mathrm{s}$} & & \multicolumn{2}{|c|}{ Number of Shots: } & 4 \\
\hline \multirow{2}{*}{\multicolumn{2}{|c|}{$\frac{\text { Std Dev: }}{\text { ZMR: }}$}} & \multicolumn{2}{|c|}{$24 \mathrm{ft} / \mathrm{s}$} & & \multicolumn{2}{|r|}{ Spread: } & $51 \mathrm{ft} / \mathrm{s}$ \\
\hline & & \multicolumn{2}{|l|}{ N/A } & & & & \\
\hline \multicolumn{2}{|c|}{ Striking Velocity } & \multirow{2}{*}{$\begin{array}{l}\text { Pitch } \\
\text { (deg) }\end{array}$} & \multirow{2}{*}{$\begin{array}{l}\text { Yaw } \\
(\mathrm{deg})\end{array}$} & \multirow{2}{*}{$\begin{array}{l}\text { Result } \\
(\mathrm{PP} / \mathrm{CP})\end{array}$} & \multirow{2}{*}{$\begin{array}{c}\text { Used for } \\
V_{50} \\
(\text { Yes/No) }\end{array}$} & \multirow{2}{*}{$\begin{array}{c}\text { Shot } \\
\text { Number }\end{array}$} & \multirow[t]{2}{*}{ Comments } \\
\hline$(\mathrm{ft} / \mathrm{s})$ & $(\mathrm{m} / \mathrm{s})$ & & & & & & \\
\hline 1524 & 464 & -- & -- & PP & No & 12547 & \\
\hline 1617 & 493 & -- & -- & $\mathrm{CP}$ & No & 12548 & \\
\hline 1632 & 497 & -- & -- & $\mathrm{CP}$ & No & 12549 & \\
\hline 1584 & 483 & -- & -- & $\mathbf{C P}$ & Yes & 12550 & \\
\hline 1537 & 468 & -- & -- & $\mathbf{P P}$ & Yes & 12551 & \\
\hline 1584 & 483 & -- & -- & PP & Yes & 12552 & \\
\hline 1588 & 484 & -- & -- & $\mathbf{C P}$ & Yes & 12553 & \\
\hline & & & & & & & \\
\hline & & & & & & & \\
\hline & & & & & & & \\
\hline & & & & & & & \\
\hline & & & & & & & \\
\hline
\end{tabular}




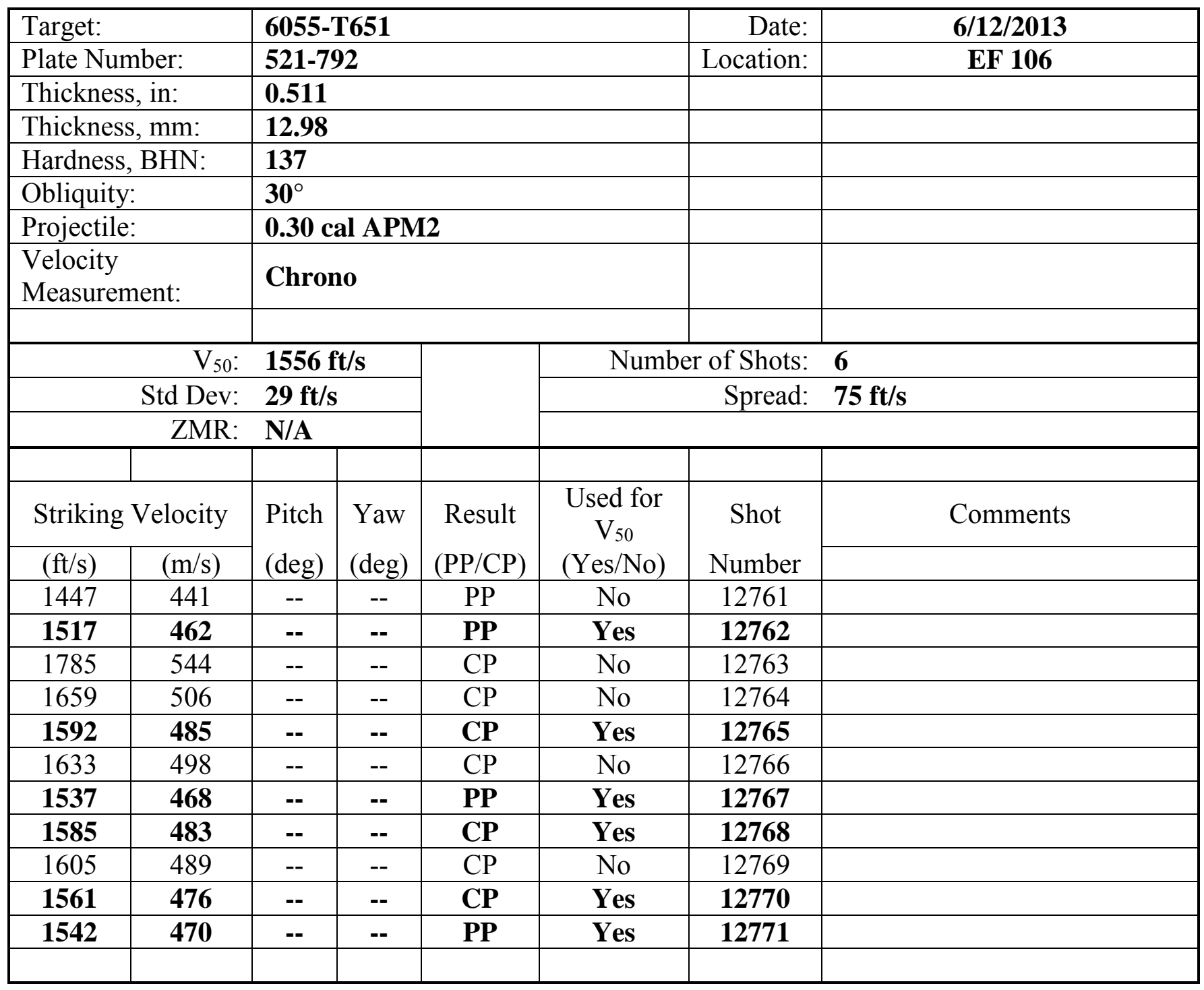




\begin{tabular}{|c|c|c|c|c|c|c|c|}
\hline \multirow{2}{*}{\multicolumn{2}{|c|}{$\begin{array}{l}\text { Target: } \\
\text { Plate Number: }\end{array}$}} & \multicolumn{4}{|c|}{ 6055-T651 } & Date: & $3 / 5 / 2013$ \\
\hline & & 521-80 & & & & Location: & EF 106 \\
\hline \multicolumn{2}{|c|}{ Thickness, in: } & \multicolumn{4}{|c|}{0.749} & & \\
\hline \multicolumn{2}{|c|}{ Thickness, mm: } & \multicolumn{4}{|l|}{19.02} & & \\
\hline \multicolumn{2}{|c|}{ Hardness, BHN: } & \multicolumn{4}{|l|}{128} & & \\
\hline \multicolumn{2}{|c|}{ Obliquity: } & \multicolumn{4}{|l|}{$30^{\circ}$} & & \\
\hline \multicolumn{2}{|c|}{ Projectile: } & \multicolumn{4}{|c|}{0.30 cal APM2 } & & \\
\hline \multicolumn{2}{|c|}{$\begin{array}{l}\text { Velocity } \\
\text { Measurement: }\end{array}$} & \multicolumn{4}{|c|}{ Chrono } & & \\
\hline \multicolumn{2}{|c|}{$\mathrm{V}_{50}:$} & \multicolumn{2}{|c|}{$2001 \mathrm{ft} / \mathrm{s}$} & & \multicolumn{2}{|c|}{ Number of Shots: } & 6 \\
\hline \multirow{2}{*}{\multicolumn{2}{|c|}{$\begin{array}{l}\text { Std Dev: } \\
\text { ZMR: }\end{array}$}} & \multicolumn{2}{|c|}{$32 \mathrm{ft} / \mathrm{s}$} & & \multicolumn{2}{|c|}{ Spread: } & $78 \mathrm{ft} / \mathrm{s}$ \\
\hline & & \multicolumn{2}{|l|}{$39 \mathrm{ft} / \mathrm{s}$} & & & & \\
\hline \multicolumn{2}{|c|}{ Striking Velocity } & \multirow{2}{*}{$\begin{array}{l}\text { Pitch } \\
(\mathrm{deg})\end{array}$} & \multirow{2}{*}{$\begin{array}{l}\text { Yaw } \\
(\mathrm{deg})\end{array}$} & \multirow{2}{*}{$\begin{array}{l}\text { Result } \\
\text { (PP/CP) }\end{array}$} & \multirow{2}{*}{$\begin{array}{c}\text { Used for } \\
\mathrm{V}_{50} \\
(\mathrm{Yes} / \mathrm{No})\end{array}$} & \multirow{2}{*}{$\begin{array}{c}\text { Shot } \\
\text { Number }\end{array}$} & \multirow[t]{2}{*}{ Comments } \\
\hline$(\mathrm{ft} / \mathrm{s})$ & $(\mathrm{m} / \mathrm{s})$ & & & & & & \\
\hline 2048 & 624 & -- & -- & CP & Yes & 12388 & \\
\hline 1973 & 601 & -- & -- & PP & Yes & 12389 & \\
\hline 2028 & 618 & -- & -- & $\mathbf{C P}$ & Yes & 12390 & \\
\hline 1979 & 603 & -- & -- & $\mathbf{P P}$ & Yes & 12391 & \\
\hline 1905 & 581 & -- & -- & PP & No & 12392 & \\
\hline 1970 & 600 & -- & -- & $\mathbf{C P}$ & Yes & 12393 & \\
\hline 2009 & 612 & -- & -- & PP & Yes & 12394 & \\
\hline & & & & & & & \\
\hline & & & & & & & \\
\hline & & & & & & & \\
\hline & & & & & & & \\
\hline & & & & & & & \\
\hline
\end{tabular}




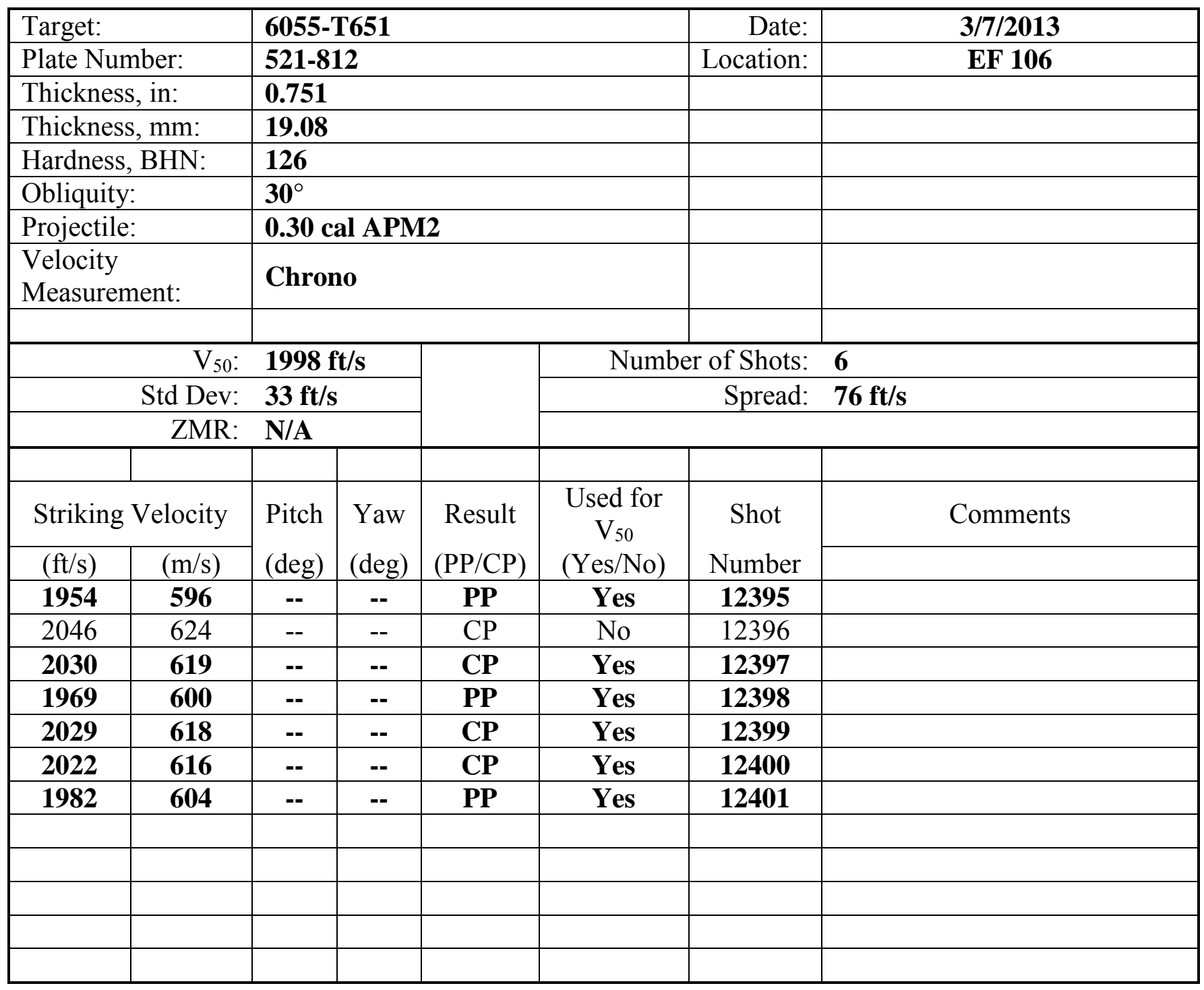




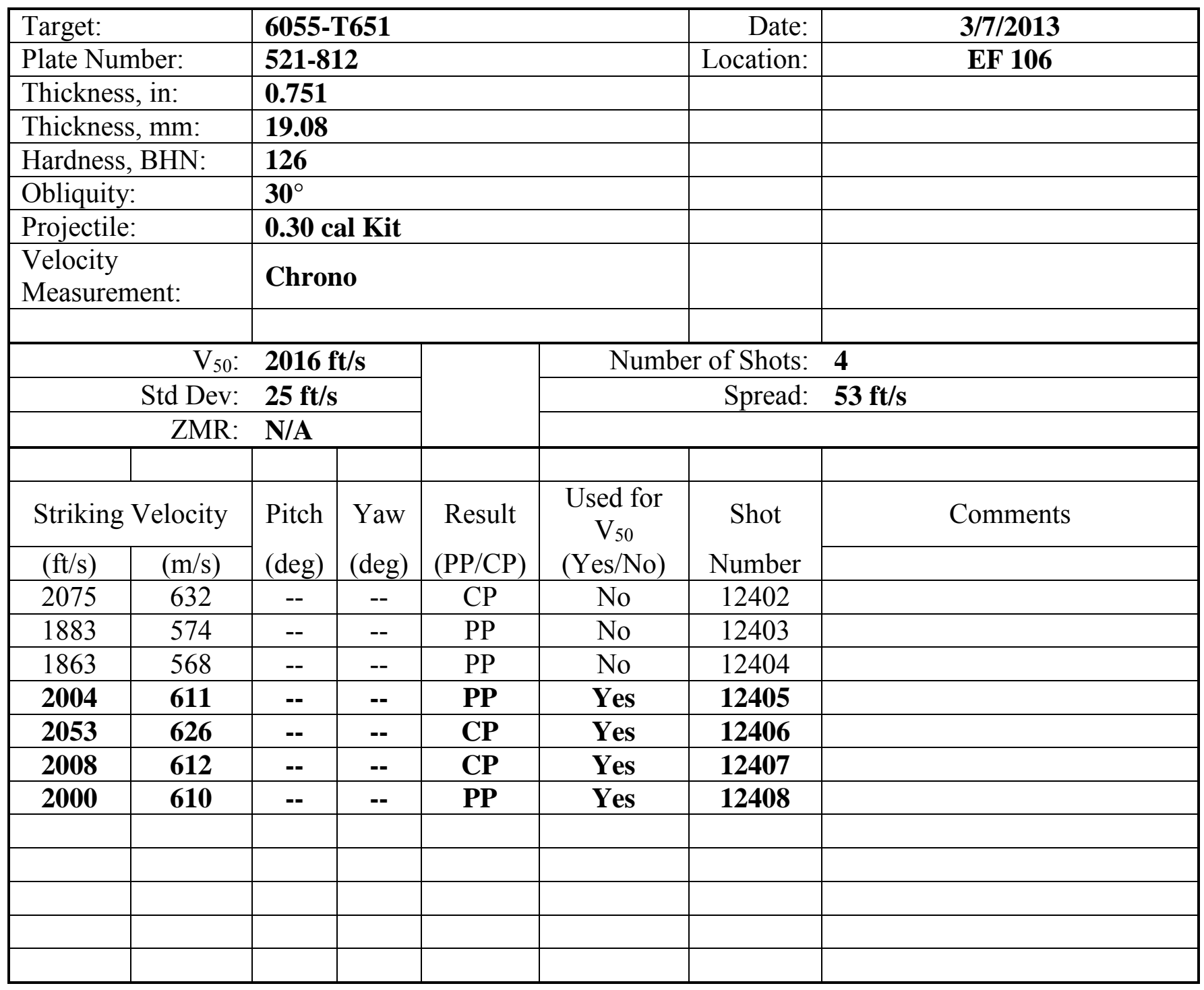




\begin{tabular}{|c|c|c|c|c|c|c|c|}
\hline \multirow{2}{*}{\multicolumn{2}{|c|}{$\begin{array}{l}\text { Target: } \\
\text { Plate Number: }\end{array}$}} & \multicolumn{4}{|c|}{ 6055-T651 } & Date: & $3 / 4 / 2013$ \\
\hline & & 521-80 & & & & Location: & EF 106 \\
\hline \multicolumn{2}{|c|}{ Thickness, in: } & \multicolumn{4}{|c|}{0.749} & & \\
\hline \multicolumn{2}{|c|}{ Thickness, mm: } & \multicolumn{4}{|l|}{19.02} & & \\
\hline \multicolumn{2}{|c|}{ Hardness, BHN: } & \multicolumn{4}{|l|}{128} & & \\
\hline \multicolumn{2}{|c|}{ Obliquity: } & \multicolumn{4}{|l|}{$\mathbf{0}^{\circ}$} & & \\
\hline \multicolumn{2}{|c|}{ Projectile: } & \multicolumn{4}{|c|}{0.30 cal APM2 } & & \\
\hline \multicolumn{2}{|c|}{$\begin{array}{l}\text { Velocity } \\
\text { Measurement: }\end{array}$} & \multicolumn{4}{|c|}{ Chrono } & & \\
\hline \multicolumn{2}{|c|}{$\mathrm{V}_{50}:$} & \multicolumn{2}{|c|}{$1770 \mathrm{ft} / \mathrm{s}$} & & \multicolumn{2}{|c|}{ Number of Shots: } & 6 \\
\hline & Std Dev: & \multicolumn{2}{|c|}{$37 \mathrm{ft} / \mathrm{s}$} & & \multicolumn{2}{|c|}{ Spread: } & $87 \mathrm{ft} / \mathrm{s}$ \\
\hline & ZMR: & \multicolumn{2}{|l|}{ N/A } & & & & \\
\hline \multicolumn{2}{|c|}{ Striking Velocity } & \multirow{2}{*}{$\begin{array}{l}\text { Pitch } \\
(\mathrm{deg})\end{array}$} & \multirow{2}{*}{$\begin{array}{l}\text { Yaw } \\
(\mathrm{deg})\end{array}$} & \multirow{2}{*}{$\begin{array}{l}\text { Result } \\
(\mathrm{PP} / \mathrm{CP})\end{array}$} & \multirow{2}{*}{$\begin{array}{c}\text { Used for } \\
\mathrm{V}_{50} \\
(\mathrm{Yes} / \mathrm{No})\end{array}$} & \multirow{2}{*}{$\begin{array}{c}\text { Shot } \\
\text { Number }\end{array}$} & \multirow[t]{2}{*}{ Comments } \\
\hline$(\mathrm{ft} / \mathrm{s})$ & $(\mathrm{m} / \mathrm{s})$ & & & & & & \\
\hline 1731 & 528 & -- & -- & $\mathbf{P P}$ & Yes & 12382 & \\
\hline 1818 & 554 & -- & -- & $\mathbf{C P}$ & Yes & 12383 & \\
\hline 1746 & 532 & -- & -- & $\mathbf{P P}$ & Yes & 12384 & \\
\hline 1797 & 548 & -- & -- & $\mathbf{C P}$ & Yes & 12385 & \\
\hline 1734 & 528 & -- & -- & $\mathbf{P P}$ & Yes & 12386 & \\
\hline 1791 & 546 & -- & -- & CP & Yes & 12387 & \\
\hline & & & & & & & \\
\hline & & & & & & & \\
\hline & & & & & & & \\
\hline & & & & & & & \\
\hline & & & & & & & \\
\hline & & & & & & & \\
\hline
\end{tabular}




\begin{tabular}{|c|c|c|c|c|c|c|c|}
\hline \multirow{2}{*}{\multicolumn{2}{|c|}{$\begin{array}{l}\text { Target: } \\
\text { Plate Number: }\end{array}$}} & \multicolumn{4}{|c|}{ 6055-T651 } & Date: & $2 / 28 / 2013$ \\
\hline & & \multicolumn{4}{|c|}{ 521-812 } & Location: & EF 106 \\
\hline \multicolumn{2}{|c|}{ Thickness, in: } & \multirow{2}{*}{\multicolumn{4}{|c|}{$\frac{0.751}{1908}$}} & & \\
\hline \multicolumn{2}{|c|}{ Thickness, mm: } & & & & & & \\
\hline \multicolumn{2}{|c|}{ Hardness, BHN: } & \multicolumn{4}{|l|}{126} & & \\
\hline \multicolumn{2}{|c|}{ Obliquity: } & \multicolumn{4}{|l|}{$0^{\circ}$} & & \\
\hline \multicolumn{2}{|c|}{ Projectile: } & \multicolumn{4}{|c|}{0.30 cal APM2 } & & \\
\hline \multicolumn{2}{|c|}{\begin{tabular}{|l|} 
Velocity \\
Measurement:
\end{tabular}} & \multicolumn{4}{|c|}{ Chrono } & & \\
\hline \multicolumn{2}{|r|}{$\mathrm{V}_{50}$ : } & \multicolumn{2}{|c|}{$1762 \mathrm{ft} / \mathrm{s}$} & & \multicolumn{2}{|c|}{ Number of Shots: } & 4 \\
\hline \multirow{2}{*}{\multicolumn{2}{|c|}{$\begin{array}{r}\text { Std Dev: } \\
\text { ZMR: }\end{array}$}} & \multicolumn{2}{|c|}{$20 \mathrm{ft} / \mathrm{s}$} & & \multicolumn{2}{|c|}{ Spread: } & $45 \mathrm{ft} / \mathrm{s}$ \\
\hline & & \multicolumn{2}{|l|}{ N/A } & & & & \\
\hline \multicolumn{2}{|c|}{ Striking Velocity } & \multirow{2}{*}{$\begin{array}{l}\text { Pitch } \\
\text { (deg) }\end{array}$} & \multirow{2}{*}{$\begin{array}{l}\text { Yaw } \\
(\mathrm{deg})\end{array}$} & \multirow{2}{*}{$\begin{array}{c}\text { Result } \\
\text { (PP/CP) }\end{array}$} & \multirow{2}{*}{$\begin{array}{c}\text { Used for } \\
\mathrm{V}_{50} \\
(\mathrm{Yes} / \mathrm{No})\end{array}$} & \multirow{2}{*}{$\begin{array}{c}\text { Shot } \\
\text { Number }\end{array}$} & \multirow[t]{2}{*}{ Comments } \\
\hline$(\mathrm{ft} / \mathrm{s})$ & $(\mathrm{m} / \mathrm{s})$ & & & & & & \\
\hline 1765 & 538 & -- & -- & PP & Yes & 12369 & \\
\hline 1822 & 555 & -- & -- & $\mathrm{CP}$ & No & 12370 & \\
\hline 1777 & 542 & -- & -- & CP & Yes & 12371 & \\
\hline 1732 & 528 & -- & -- & PP & Yes & 12372 & \\
\hline 1772 & 540 & -- & -- & $\mathbf{C P}$ & Yes & 12373 & \\
\hline & & & & & & & \\
\hline & & & & & & & \\
\hline & & & & & & & \\
\hline & & & & & & & \\
\hline & & & & & & & \\
\hline & & & & & & & \\
\hline & & & & & & & \\
\hline
\end{tabular}




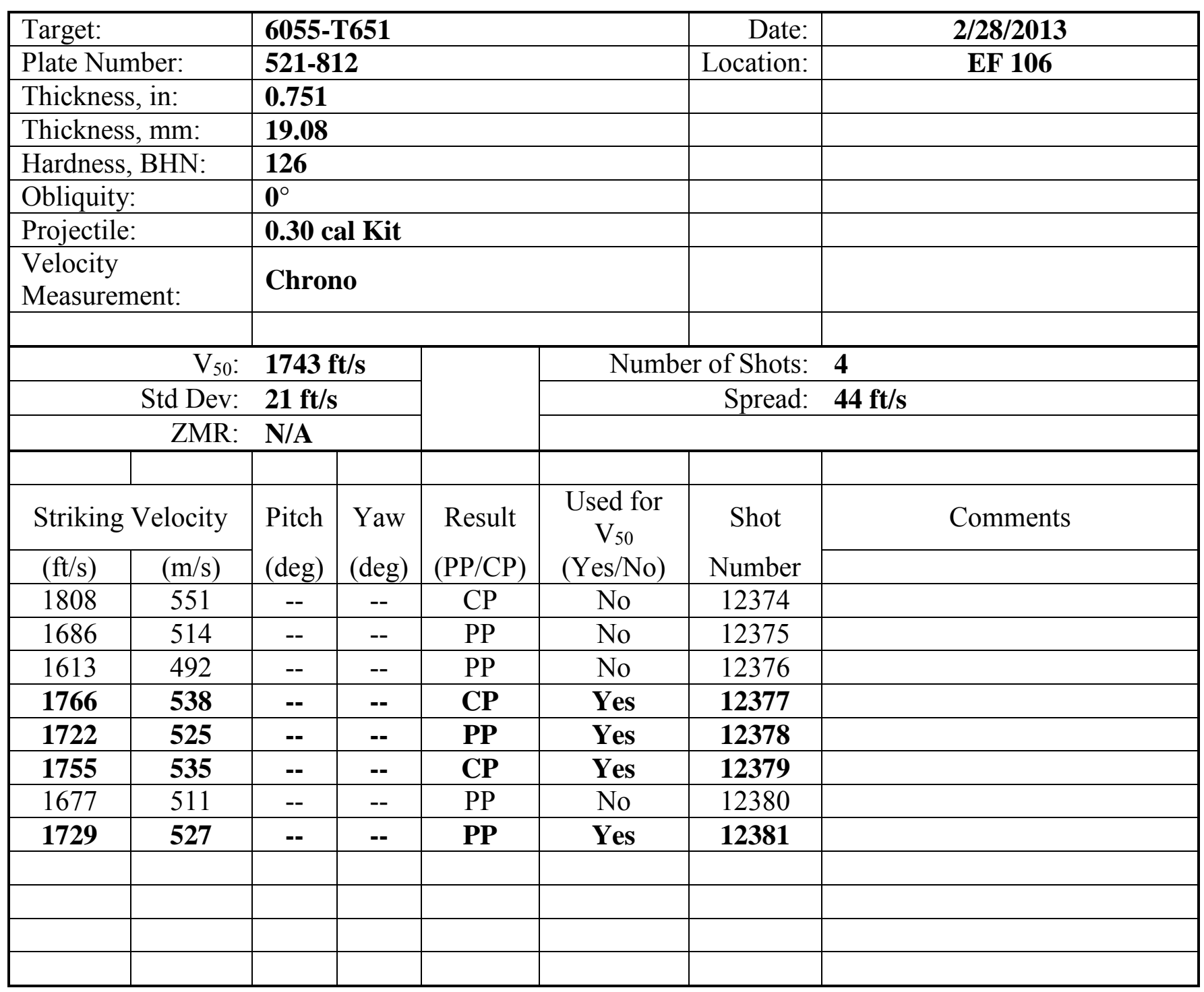




\begin{tabular}{|c|c|c|c|c|c|c|c|}
\hline \multirow{2}{*}{\multicolumn{2}{|c|}{$\begin{array}{l}\text { Target: } \\
\text { Plate Number: }\end{array}$}} & \multicolumn{4}{|c|}{ 6055-T651 } & Date: & $2 / 26 / 2013$ \\
\hline & & $521-8$ & & & & Location: & EF 106 \\
\hline \multicolumn{2}{|c|}{ Thickness, in: } & \multicolumn{4}{|c|}{0.994} & & \\
\hline \multicolumn{2}{|c|}{ Thickness, mm: } & \multicolumn{4}{|c|}{25.25} & & \\
\hline \multicolumn{2}{|c|}{ Hardness, BHN: } & \multicolumn{4}{|l|}{118} & & \\
\hline \multicolumn{2}{|c|}{ Obliquity: } & \multicolumn{4}{|l|}{$\mathbf{0}^{\circ}$} & & \\
\hline \multicolumn{2}{|c|}{ Projectile: } & \multicolumn{4}{|c|}{0.30 cal APM2 } & & \\
\hline \multicolumn{2}{|c|}{\begin{tabular}{|l|} 
Velocity \\
Measurement:
\end{tabular}} & \multicolumn{4}{|c|}{ Chrono } & & \\
\hline \multicolumn{2}{|c|}{$\mathrm{V}_{50}:$} & \multicolumn{2}{|c|}{$2028 \mathrm{ft} / \mathrm{s}$} & & \multicolumn{2}{|c|}{ Number of Shots: } & 6 \\
\hline \multirow{2}{*}{\multicolumn{2}{|c|}{$\begin{array}{l}\text { Std Dev: } \\
\text { ZMR: }\end{array}$}} & \multicolumn{2}{|c|}{$34 \mathrm{ft} / \mathrm{s}$} & & \multicolumn{2}{|c|}{ Spread: } & $79 \mathrm{ft} / \mathrm{s}$ \\
\hline & & \multicolumn{2}{|c|}{$28 \mathrm{ft} / \mathrm{s}$} & & & & \\
\hline \multicolumn{2}{|c|}{ Striking Velocity } & \multirow{2}{*}{$\begin{array}{l}\text { Pitch } \\
\text { (deg) }\end{array}$} & \multirow{2}{*}{$\begin{array}{l}\text { Yaw } \\
(\mathrm{deg})\end{array}$} & \multirow{2}{*}{$\begin{array}{c}\text { Result } \\
\text { (PP/CP) }\end{array}$} & \multirow{2}{*}{$\begin{array}{c}\text { Used for } \\
\mathrm{V}_{50} \\
(\mathrm{Yes} / \mathrm{No})\end{array}$} & \multirow{2}{*}{$\begin{array}{c}\text { Shot } \\
\text { Number }\end{array}$} & \multirow[t]{2}{*}{ Comments } \\
\hline$(\mathrm{ft} / \mathrm{s})$ & $(\mathrm{m} / \mathrm{s})$ & & & & & & \\
\hline 1991 & 607 & -- & -- & PP & Yes & 12348 & \\
\hline 2014 & 614 & -- & -- & PP & Yes & 12349 & \\
\hline 2081 & 634 & -- & -- & $\mathrm{CP}$ & No & 12350 & \\
\hline 1997 & 609 & -- & -- & $\mathbf{C P}$ & Yes & 12351 & \\
\hline 2070 & 631 & -- & -- & $\mathbf{C P}$ & Yes & 12352 & \\
\hline 2068 & 630 & -- & -- & CP & Yes & 12353 & \\
\hline 2025 & 617 & -- & -- & PP & Yes & 12354 & \\
\hline & & & & & & & \\
\hline & & & & & & & \\
\hline & & & & & & & \\
\hline & & & & & & & \\
\hline & & & & & & & \\
\hline
\end{tabular}




\begin{tabular}{|c|c|c|c|c|c|c|c|}
\hline \multirow{2}{*}{\multicolumn{2}{|c|}{$\begin{array}{l}\text { Target: } \\
\text { Plate Number: }\end{array}$}} & \multicolumn{4}{|c|}{ 6055-T651 } & Date: & $2 / 25 / 2013$ \\
\hline & & 521-82 & & & & Location: & EF 106 \\
\hline \multicolumn{2}{|c|}{ Thickness, in: } & \multicolumn{4}{|c|}{0.994} & & \\
\hline \multicolumn{2}{|c|}{ Thickness, mm: } & \multicolumn{4}{|l|}{25.25} & & \\
\hline \multicolumn{2}{|c|}{ Hardness, BHN: } & \multicolumn{4}{|l|}{118} & & \\
\hline \multicolumn{2}{|c|}{ Obliquity: } & \multicolumn{4}{|l|}{$0^{\circ}$} & & \\
\hline \multicolumn{2}{|c|}{ Projectile: } & \multicolumn{4}{|c|}{0.30 cal Kit } & & \\
\hline \multicolumn{2}{|c|}{$\begin{array}{l}\text { Velocity } \\
\text { Measurement: }\end{array}$} & \multicolumn{4}{|c|}{ Chrono } & & \\
\hline \multicolumn{2}{|c|}{$\mathrm{V}_{50}:$} & \multicolumn{2}{|c|}{$2033 \mathrm{ft} / \mathrm{s}$} & & \multicolumn{2}{|c|}{ Number of Shots: } & 4 \\
\hline & Std Dev: & \multicolumn{2}{|c|}{$21 \mathrm{ft} / \mathrm{s}$} & & \multicolumn{2}{|c|}{ Spread: } & $52 \mathrm{ft} / \mathrm{s}$ \\
\hline & ZMR: & \multicolumn{2}{|l|}{ N/A } & & & & \\
\hline \multicolumn{2}{|c|}{ Striking Velocity } & \multirow{2}{*}{$\begin{array}{l}\text { Pitch } \\
(\mathrm{deg})\end{array}$} & \multirow{2}{*}{$\begin{array}{l}\text { Yaw } \\
(\mathrm{deg})\end{array}$} & \multirow{2}{*}{$\begin{array}{l}\text { Result } \\
(\mathrm{PP} / \mathrm{CP})\end{array}$} & \multirow{2}{*}{$\begin{array}{c}\text { Used for } \\
\mathrm{V}_{50} \\
(\mathrm{Yes} / \mathrm{No})\end{array}$} & \multirow{2}{*}{$\begin{array}{c}\text { Shot } \\
\text { Number }\end{array}$} & \multirow[t]{2}{*}{ Comments } \\
\hline$(\mathrm{ft} / \mathrm{s})$ & $(\mathrm{m} / \mathrm{s})$ & & & & & & \\
\hline 2191 & 668 & -- & -- & $\mathrm{CP}$ & No & 12341 & \\
\hline 2037 & 621 & -- & -- & $\mathbf{C P}$ & Yes & 12342 & \\
\hline 1899 & 579 & -- & -- & PP & No & 12343 & \\
\hline 2005 & 611 & -- & -- & $\mathbf{P P}$ & Yes & 12344 & \\
\hline 1937 & 590 & -- & -- & PP & No & 12345 & \\
\hline 2034 & 620 & -- & -- & $\mathbf{P P}$ & Yes & 12346 & \\
\hline 2057 & 627 & -- & -- & CP & Yes & 12347 & \\
\hline & & & & & & & \\
\hline & & & & & & & \\
\hline & & & & & & & \\
\hline & & & & & & & \\
\hline & & & & & & & \\
\hline
\end{tabular}




\begin{tabular}{|c|c|c|c|c|c|c|c|}
\hline \multirow{2}{*}{\multicolumn{2}{|c|}{$\begin{array}{l}\text { Target: } \\
\text { Plate Number: }\end{array}$}} & \multicolumn{4}{|c|}{ 6055-T651 } & Date: & $2 / 26 / 2013$ \\
\hline & & $521-8$ & & & & Location: & EF 106 \\
\hline \multicolumn{2}{|c|}{ Thickness, in: } & \multicolumn{4}{|c|}{1.011} & & \\
\hline \multicolumn{2}{|c|}{ Thickness, mm: } & \multicolumn{4}{|l|}{25.68} & & \\
\hline \multicolumn{2}{|c|}{ Hardness, BHN: } & \multicolumn{4}{|l|}{124} & & \\
\hline \multicolumn{2}{|c|}{ Obliquity: } & \multicolumn{4}{|l|}{$0^{\circ}$} & & \\
\hline \multicolumn{2}{|c|}{ Projectile: } & \multicolumn{4}{|c|}{0.30 cal APM2 } & & \\
\hline \multicolumn{2}{|c|}{\begin{tabular}{|l|} 
Velocity \\
Measurement:
\end{tabular}} & \multicolumn{4}{|c|}{ Chrono } & & \\
\hline \multicolumn{2}{|c|}{$\mathrm{V}_{50}:$} & \multicolumn{2}{|c|}{$2079 \mathrm{ft} / \mathrm{s}$} & & \multicolumn{2}{|c|}{ Number of Shots: } & 6 \\
\hline \multirow{2}{*}{\multicolumn{2}{|c|}{$\begin{array}{l}\text { Std Dev: } \\
\text { ZMR: }\end{array}$}} & \multicolumn{2}{|c|}{$31 \mathrm{ft} / \mathrm{s}$} & & \multicolumn{2}{|c|}{ Spread: } & $83 \mathrm{ft} / \mathrm{s}$ \\
\hline & & \multicolumn{2}{|c|}{$17 \mathrm{ft} / \mathrm{s}$} & & & & \\
\hline \multicolumn{2}{|c|}{ Striking Velocity } & \multirow{2}{*}{$\begin{array}{l}\text { Pitch } \\
\text { (deg) }\end{array}$} & \multirow{2}{*}{$\begin{array}{l}\text { Yaw } \\
(\mathrm{deg})\end{array}$} & \multirow{2}{*}{$\begin{array}{l}\text { Result } \\
\text { (PP/CP) }\end{array}$} & \multirow{2}{*}{$\begin{array}{c}\text { Used for } \\
V_{50} \\
(\text { Yes/No) }\end{array}$} & \multirow{2}{*}{$\begin{array}{c}\text { Shot } \\
\text { Number }\end{array}$} & \multirow[t]{2}{*}{ Comments } \\
\hline$(\mathrm{ft} / \mathrm{s})$ & $(\mathrm{m} / \mathrm{s})$ & & & & & & \\
\hline 2021 & 616 & -- & -- & PP & No & 12355 & \\
\hline 2114 & 644 & -- & -- & $\mathbf{C P}$ & Yes & 12356 & \\
\hline 2106 & 642 & -- & -- & $\mathbf{C P}$ & Yes & 12357 & \\
\hline 2031 & 619 & -- & -- & $\mathbf{P P}$ & Yes & 12358 & \\
\hline 2011 & 613 & -- & -- & PP & No & 12359 & \\
\hline 2062 & 628 & -- & -- & PP & Yes & 12360 & \\
\hline 2072 & 632 & -- & -- & CP & Yes & 12361 & \\
\hline 2089 & 637 & -- & -- & PP & Yes & 12362 & \\
\hline & & & & & & & \\
\hline & & & & & & & \\
\hline & & & & & & & \\
\hline & & & & & & & \\
\hline
\end{tabular}




\begin{tabular}{|c|c|c|c|c|c|c|c|}
\hline \multicolumn{2}{|l|}{ Target: } & \multicolumn{4}{|c|}{ 6055-T651 } & Date: & $2 / 27 / 2013$ \\
\hline \multicolumn{2}{|c|}{ Plate Number: } & \multicolumn{4}{|c|}{$521-842$} & Location: & EF 106 \\
\hline \multicolumn{2}{|c|}{ Thickness, in: } & \multicolumn{4}{|l|}{0.999} & & \\
\hline \multicolumn{2}{|c|}{ Thickness, mm: } & \multicolumn{4}{|l|}{25.37} & & \\
\hline \multicolumn{2}{|c|}{ Hardness, BHN: } & \multicolumn{4}{|l|}{124} & & \\
\hline \multicolumn{2}{|c|}{ Obliquity: } & \multicolumn{4}{|l|}{$0^{\circ}$} & & \\
\hline \multicolumn{2}{|c|}{ Projectile: } & \multicolumn{4}{|c|}{0.30 cal APM2 } & & \\
\hline \multicolumn{2}{|c|}{$\begin{array}{l}\text { Velocity } \\
\text { Measurement: }\end{array}$} & \multicolumn{4}{|c|}{ Chrono } & & \\
\hline \multicolumn{2}{|r|}{$\mathrm{V}_{50}$ : } & \multicolumn{2}{|c|}{$2067 \mathrm{ft} / \mathrm{s}$} & & \multicolumn{2}{|c|}{ Number of Shots: } & 4 \\
\hline & Std Dev: & \multicolumn{2}{|c|}{$25 \mathrm{ft} / \mathrm{s}$} & & \multicolumn{2}{|c|}{ Spread: } & $55 \mathrm{ft} / \mathrm{s}$ \\
\hline & ZMR: & \multicolumn{2}{|l|}{ N/A } & & & & \\
\hline \multicolumn{2}{|c|}{ Striking Velocity } & \multirow{2}{*}{$\begin{array}{l}\text { Pitch } \\
\text { (deg) }\end{array}$} & \multirow{2}{*}{$\begin{array}{l}\text { Yaw } \\
(\mathrm{deg})\end{array}$} & \multirow{2}{*}{$\begin{array}{c}\text { Result } \\
\text { (PP/CP) }\end{array}$} & \multirow{2}{*}{$\begin{array}{c}\text { Used for } \\
\mathrm{V}_{50} \\
(\mathrm{Yes} / \mathrm{No})\end{array}$} & \multirow{2}{*}{$\begin{array}{c}\text { Shot } \\
\text { Number }\end{array}$} & \multirow[t]{2}{*}{ Comments } \\
\hline$(\mathrm{ft} / \mathrm{s})$ & $(\mathrm{m} / \mathrm{s})$ & & & & & & \\
\hline 2120 & 646 & -- & -- & $\mathrm{CP}$ & No & 12363 & \\
\hline 1977 & 603 & -- & -- & PP & No & 12364 & \\
\hline 2037 & 621 & -- & -- & PP & Yes & 12365 & \\
\hline 2056 & 627 & -- & -- & PP & Yes & 12366 & \\
\hline 2081 & 634 & -- & -- & $\mathbf{C P}$ & Yes & 12367 & \\
\hline 2092 & 638 & -- & -- & CP & Yes & 12368 & \\
\hline & & & & & & & \\
\hline & & & & & & & \\
\hline & & & & & & & \\
\hline & & & & & & & \\
\hline & & & & & & & \\
\hline & & & & & & & \\
\hline
\end{tabular}




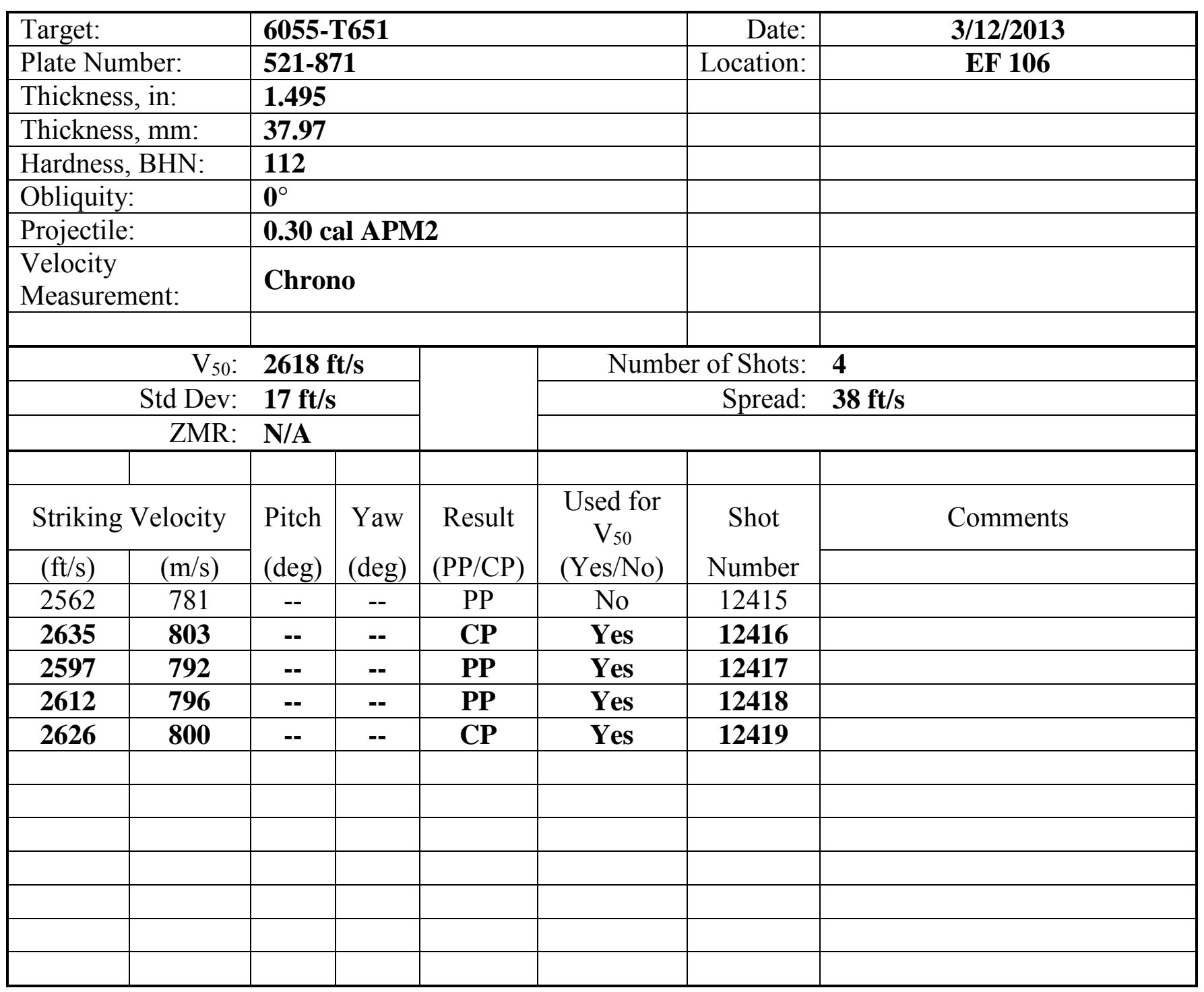




\begin{tabular}{|c|c|c|c|c|c|c|c|}
\hline \multirow{2}{*}{\multicolumn{2}{|c|}{$\begin{array}{l}\text { Target: } \\
\text { Plate Number: }\end{array}$}} & \multicolumn{4}{|c|}{ 6055-T651 } & Date: & $3 / 11 / 2013$ \\
\hline & & $521-8$ & & & & Location: & EF 106 \\
\hline \multicolumn{2}{|c|}{ Thickness, in: } & \multicolumn{4}{|c|}{1.495} & & \\
\hline \multicolumn{2}{|c|}{ Thickness, mm: } & \multicolumn{4}{|l|}{37.97} & & \\
\hline \multicolumn{2}{|c|}{ Hardness, BHN: } & \multicolumn{4}{|l|}{112} & & \\
\hline \multicolumn{2}{|c|}{ Obliquity: } & \multicolumn{4}{|l|}{$0^{\circ}$} & & \\
\hline \multicolumn{2}{|c|}{ Projectile: } & \multicolumn{4}{|c|}{0.30 cal Kit } & & \\
\hline \multicolumn{2}{|c|}{\begin{tabular}{|l|} 
Velocity \\
Measurement:
\end{tabular}} & \multicolumn{4}{|c|}{ Chrono } & & \\
\hline \multicolumn{2}{|r|}{$\mathrm{V}_{50}$ : } & \multicolumn{2}{|c|}{$2626 \mathrm{ft} / \mathrm{s}$} & & \multicolumn{2}{|c|}{ Number of Shots: } & 4 \\
\hline \multirow{2}{*}{\multicolumn{2}{|c|}{$\begin{array}{r}\text { Std Dev: } \\
\text { ZMR: }\end{array}$}} & \multicolumn{2}{|c|}{$28 \mathrm{ft} / \mathrm{s}$} & & \multicolumn{2}{|c|}{ Spread: } & $55 \mathrm{ft} / \mathrm{s}$ \\
\hline & & \multicolumn{2}{|l|}{ N/A } & & & & \\
\hline \multicolumn{2}{|c|}{ Striking Velocity } & \multirow{2}{*}{$\begin{array}{l}\text { Pitch } \\
\text { (deg) }\end{array}$} & \multirow{2}{*}{$\begin{array}{l}\text { Yaw } \\
\text { (deg) }\end{array}$} & \multirow{2}{*}{$\begin{array}{l}\text { Result } \\
(\mathrm{PP} / \mathrm{CP})\end{array}$} & \multirow{2}{*}{$\begin{array}{c}\text { Used for } \\
\mathrm{V}_{50} \\
(\mathrm{Yes} / \mathrm{No})\end{array}$} & \multirow{2}{*}{$\begin{array}{c}\text { Shot } \\
\text { Number }\end{array}$} & \multirow[t]{2}{*}{ Comments } \\
\hline$(\mathrm{ft} / \mathrm{s})$ & $(\mathrm{m} / \mathrm{s})$ & & & & & & \\
\hline 2757 & 840 & -- & -- & $\mathrm{CP}$ & No & 12409 & \\
\hline 2711 & 826 & -- & -- & $\mathrm{CP}$ & No & 12410 & \\
\hline 2600 & 792 & -- & -- & PP & Yes & 12411 & \\
\hline 2655 & 809 & -- & -- & $\mathbf{C P}$ & Yes & 12412 & \\
\hline 2603 & 793 & -- & -- & PP & Yes & 12413 & \\
\hline 2645 & 806 & -- & -- & CP & Yes & 12414 & \\
\hline & & & & & & & \\
\hline & & & & & & & \\
\hline & & & & & & & \\
\hline & & & & & & & \\
\hline & & & & & & & \\
\hline & & & & & & & \\
\hline
\end{tabular}




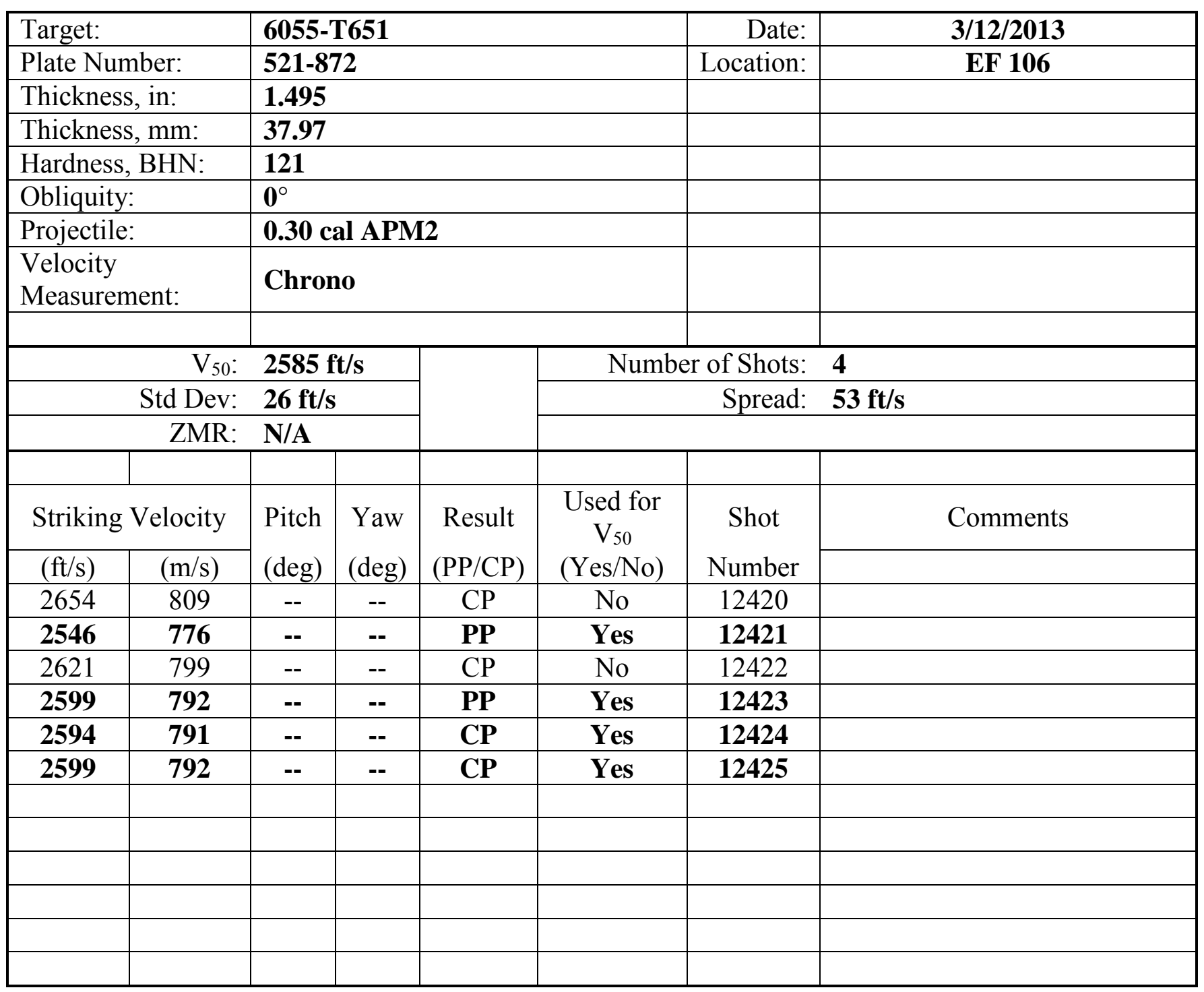




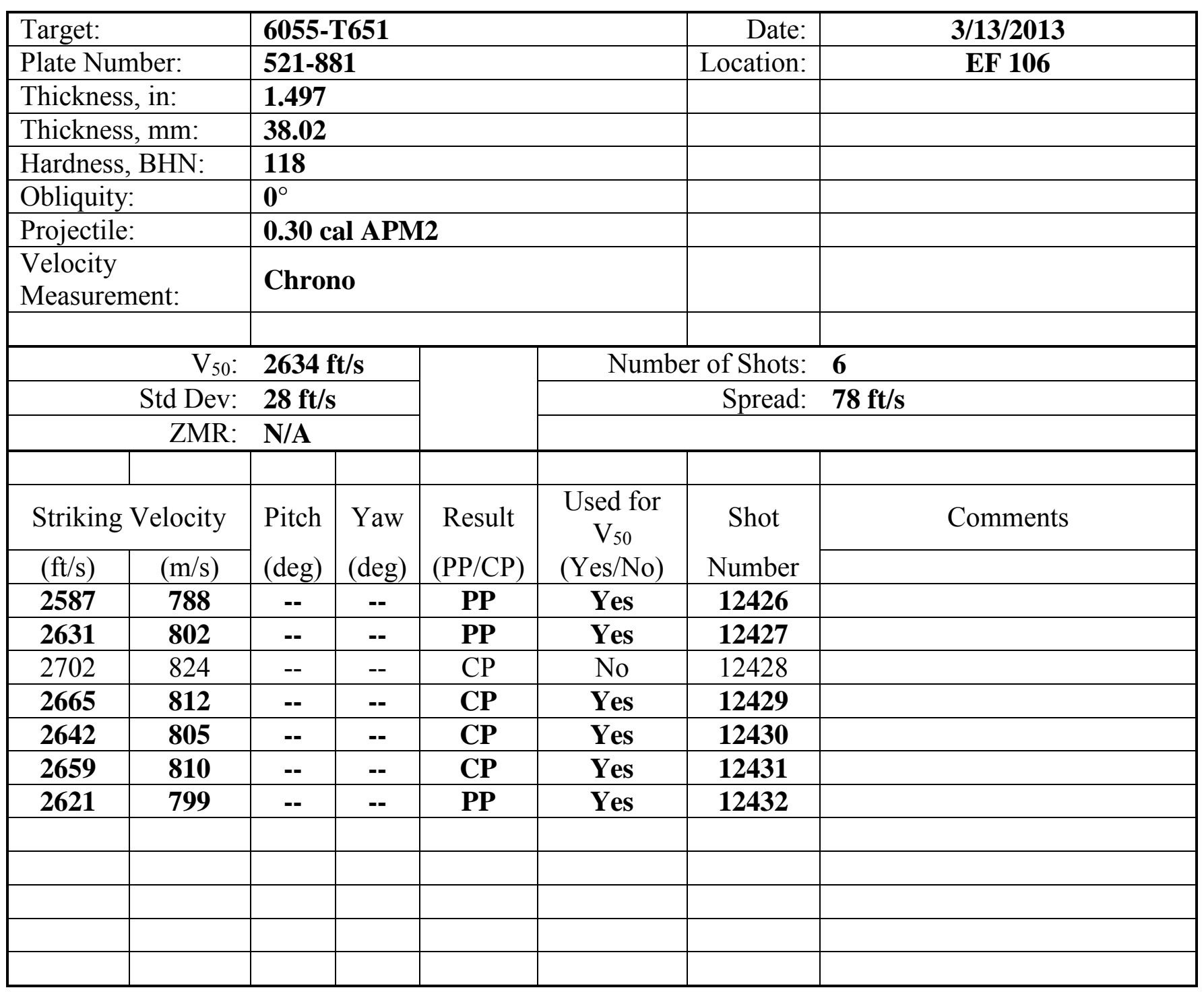




\begin{tabular}{|c|c|c|c|c|c|c|c|}
\hline \multicolumn{2}{|l|}{ Target: } & \multicolumn{4}{|c|}{ 6055-T651 } & Date: & $3 / 13 / 2013$ \\
\hline \multicolumn{2}{|c|}{ Plate Number: } & \multicolumn{4}{|c|}{$521-882$} & Location: & EF 106 \\
\hline \multicolumn{2}{|c|}{ Thickness, in: } & \multicolumn{4}{|l|}{1.500} & & \\
\hline \multicolumn{2}{|c|}{ Thickness, mm: } & \multicolumn{4}{|l|}{38.10} & & \\
\hline \multicolumn{2}{|c|}{ Hardness, BHN: } & \multicolumn{4}{|l|}{121} & & \\
\hline \multicolumn{2}{|c|}{ Obliquity: } & \multicolumn{4}{|l|}{$0^{\circ}$} & & \\
\hline \multicolumn{2}{|c|}{ Projectile: } & \multicolumn{4}{|c|}{0.30 cal APM2 } & & \\
\hline \multicolumn{2}{|c|}{\begin{tabular}{|l|} 
Velocity \\
Measurement:
\end{tabular}} & \multicolumn{4}{|c|}{ Chrono } & & \\
\hline \multicolumn{2}{|r|}{$\mathrm{V}_{50}$ : } & \multicolumn{2}{|c|}{$2654 \mathrm{ft} / \mathrm{s}$} & & \multicolumn{2}{|c|}{ Number of Shots: } & 4 \\
\hline & Std Dev: & \multicolumn{2}{|c|}{$25 \mathrm{ft} / \mathrm{s}$} & & \multicolumn{2}{|c|}{ Spread: } & $58 \mathrm{ft} / \mathrm{s}$ \\
\hline & ZMR: & \multicolumn{2}{|l|}{ N/A } & & & & \\
\hline \multicolumn{2}{|c|}{ Striking Velocity } & \multirow{2}{*}{$\begin{array}{l}\text { Pitch } \\
\text { (deg) }\end{array}$} & \multirow{2}{*}{$\begin{array}{l}\text { Yaw } \\
(\mathrm{deg})\end{array}$} & \multirow{2}{*}{$\begin{array}{c}\text { Result } \\
\text { (PP/CP) }\end{array}$} & \multirow{2}{*}{$\begin{array}{c}\text { Used for } \\
\mathrm{V}_{50} \\
(\mathrm{Yes} / \mathrm{No})\end{array}$} & \multirow{2}{*}{$\begin{array}{c}\text { Shot } \\
\text { Number }\end{array}$} & \multirow[t]{2}{*}{ Comments } \\
\hline$(\mathrm{ft} / \mathrm{s})$ & $(\mathrm{m} / \mathrm{s})$ & & & & & & \\
\hline 2641 & 805 & -- & -- & PP & Yes & 12433 & \\
\hline 2723 & 830 & -- & -- & $\mathrm{CP}$ & No & 12434 & \\
\hline 2689 & 820 & -- & -- & $\mathbf{C P}$ & Yes & 12435 & \\
\hline 2654 & 809 & -- & -- & $\mathbf{C P}$ & Yes & 12436 & \\
\hline 2563 & 781 & -- & -- & PP & No & 12437 & \\
\hline 2631 & 802 & -- & -- & PP & Yes & 12438 & \\
\hline & & & & & & & \\
\hline & & & & & & & \\
\hline & & & & & & & \\
\hline & & & & & & & \\
\hline & & & & & & & \\
\hline & & & & & & & \\
\hline
\end{tabular}




\begin{tabular}{|c|c|c|c|c|c|c|c|}
\hline \multicolumn{2}{|l|}{ Target: } & \multicolumn{4}{|c|}{ 6055-T651 } & Date: & $6 / 11 / 2013$ \\
\hline \multicolumn{2}{|c|}{ Plate Number: } & \multicolumn{4}{|c|}{$521-892$} & Location: & EF 106 \\
\hline \multicolumn{2}{|c|}{ Thickness, in: } & \multicolumn{4}{|l|}{1.498} & & \\
\hline \multicolumn{2}{|c|}{ Thickness, mm: } & \multicolumn{4}{|l|}{38.05} & & \\
\hline \multicolumn{2}{|c|}{ Hardness, BHN: } & \multicolumn{4}{|l|}{109} & & \\
\hline \multicolumn{2}{|c|}{ Obliquity: } & \multicolumn{4}{|l|}{$0^{\circ}$} & & \\
\hline \multicolumn{2}{|c|}{ Projectile: } & \multicolumn{4}{|c|}{0.30 cal APM2 } & & \\
\hline \multicolumn{2}{|c|}{$\begin{array}{l}\text { Velocity } \\
\text { Measurement: }\end{array}$} & \multicolumn{4}{|c|}{ Chrono } & & \\
\hline \multicolumn{2}{|r|}{$\mathrm{V}_{50}$ : } & \multicolumn{2}{|c|}{$2651 \mathrm{ft} / \mathrm{s}$} & & \multicolumn{2}{|c|}{ Number of Shots: } & 6 \\
\hline \multirow{2}{*}{\multicolumn{2}{|c|}{$\begin{array}{r}\text { Std Dev: } \\
\text { ZMR: }\end{array}$}} & \multicolumn{2}{|c|}{$27 \mathrm{ft} / \mathrm{s}$} & & \multicolumn{2}{|c|}{ Spread: } & $76 \mathrm{ft} / \mathrm{s}$ \\
\hline & & \multicolumn{2}{|c|}{$25 \mathrm{ft} / \mathrm{s}$} & & & & \\
\hline \multicolumn{2}{|c|}{ Striking Velocity } & \multirow{2}{*}{$\begin{array}{l}\text { Pitch } \\
\text { (deg) }\end{array}$} & \multirow{2}{*}{$\begin{array}{l}\text { Yaw } \\
\text { (deg) }\end{array}$} & \multirow{2}{*}{$\begin{array}{l}\text { Result } \\
(\mathrm{PP} / \mathrm{CP})\end{array}$} & \multirow{2}{*}{$\begin{array}{c}\text { Used for } \\
\mathrm{V}_{50} \\
(\mathrm{Yes} / \mathrm{No})\end{array}$} & \multirow{2}{*}{$\begin{array}{c}\text { Shot } \\
\text { Number }\end{array}$} & \multirow[t]{2}{*}{ Comments } \\
\hline$(\mathrm{ft} / \mathrm{s})$ & $(\mathrm{m} / \mathrm{s})$ & & & & & & \\
\hline 2661 & 811 & -- & -- & PP & Yes & 12753 & \\
\hline 2803 & 854 & -- & -- & $\mathrm{CP}$ & No & 12754 & \\
\hline 2711 & 826 & -- & -- & $\mathrm{CP}$ & No & 12755 & \\
\hline 2683 & 818 & -- & -- & $\mathbf{C P}$ & Yes & 12756 & \\
\hline 2607 & 795 & -- & -- & PP & Yes & 12757 & \\
\hline 2670 & 814 & -- & -- & CP & Yes & 12758 & \\
\hline 2636 & 803 & -- & -- & CP & Yes & 12759 & \\
\hline 2649 & 807 & -- & -- & PP & Yes & 12760 & \\
\hline & & & & & & & \\
\hline & & & & & & & \\
\hline & & & & & & & \\
\hline & & & & & & & \\
\hline
\end{tabular}




\subsection{0-cal APM2}

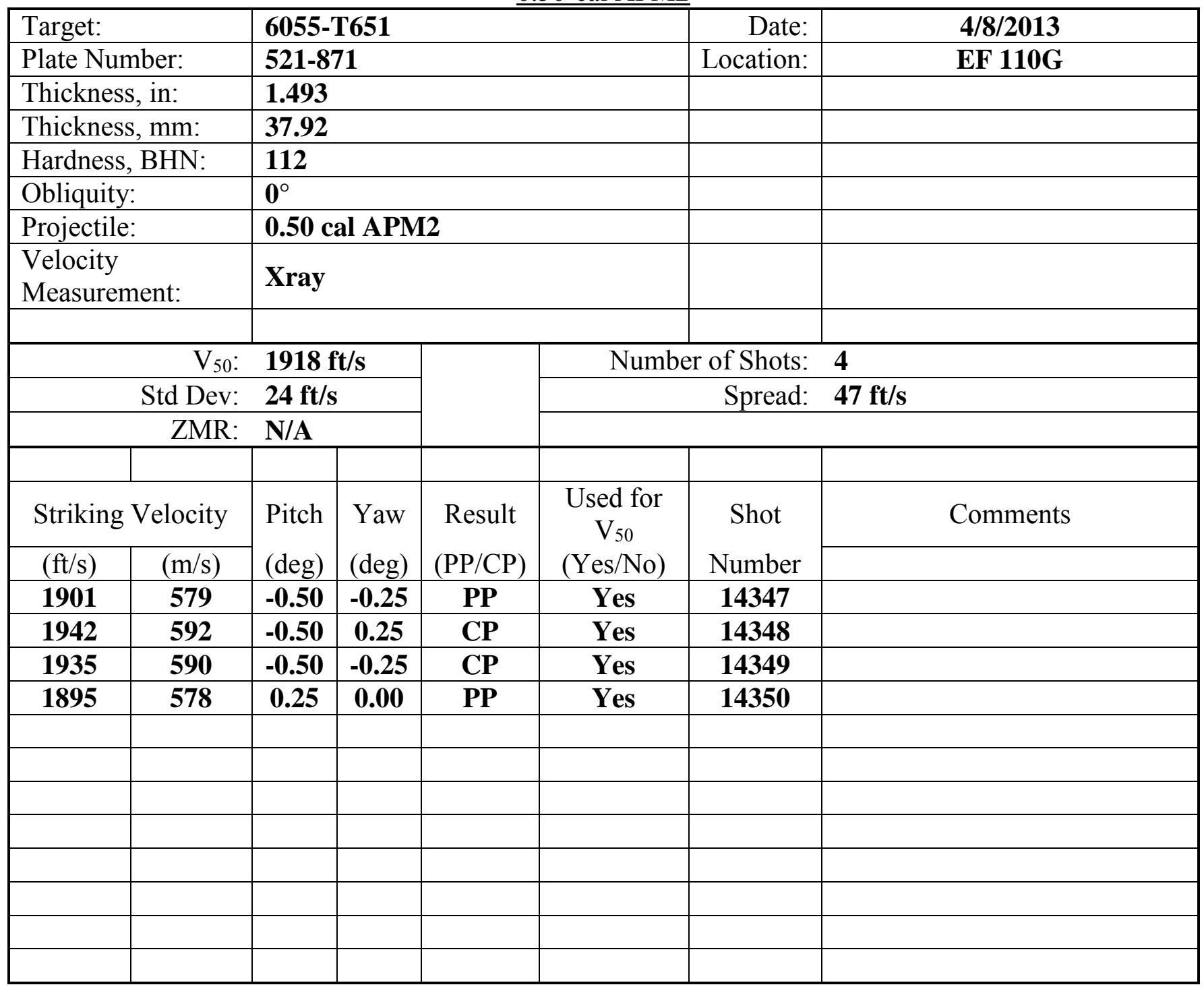




\begin{tabular}{|c|c|c|c|c|c|c|c|}
\hline \multicolumn{2}{|c|}{ Target: } & \multicolumn{4}{|c|}{ 6055-T651 } & Date: & 4/9/2013 \\
\hline \multicolumn{2}{|c|}{ Plate Number: } & \multicolumn{4}{|c|}{ 521-872 } & Location: & EF 110G \\
\hline \multicolumn{2}{|c|}{ Thickness, in: } & \multicolumn{4}{|c|}{1.494} & & \\
\hline \multicolumn{2}{|c|}{ Thickness, mm: } & \multicolumn{4}{|l|}{37.95} & & \\
\hline \multicolumn{2}{|c|}{ Hardness, BHN: } & \multicolumn{4}{|l|}{112} & & \\
\hline \multicolumn{2}{|c|}{ Obliquity: } & \multicolumn{4}{|l|}{$0^{\circ}$} & & \\
\hline \multicolumn{2}{|c|}{ Projectile: } & \multicolumn{4}{|c|}{0.50 cal APM2 } & & \\
\hline \multicolumn{2}{|c|}{$\begin{array}{l}\text { Velocity } \\
\text { Measurement: }\end{array}$} & \multicolumn{4}{|l|}{ Xray } & & \\
\hline & $\mathrm{V}_{50}$ & \multicolumn{2}{|c|}{$1915 \mathrm{ft} / \mathrm{s}$} & & \multicolumn{2}{|c|}{ Number of Shots: } & 4 \\
\hline & Std Dev & \multicolumn{2}{|c|}{$24 \mathrm{ft} / \mathrm{s}$} & & \multicolumn{2}{|r|}{ Spread: } & $56 \mathrm{ft} / \mathrm{s}$ \\
\hline & ZMR & \multicolumn{2}{|l|}{ N/A } & & & & \\
\hline \multicolumn{2}{|c|}{ Striking Velocity } & \multirow{2}{*}{$\begin{array}{l}\text { Pitch } \\
\text { (deg) }\end{array}$} & \multirow{2}{*}{$\begin{array}{l}\text { Yaw } \\
(\mathrm{deg})\end{array}$} & \multirow{2}{*}{$\begin{array}{l}\text { Result } \\
\text { (PP/CP) }\end{array}$} & \multirow{2}{*}{$\begin{array}{c}\text { Used for } \\
V_{50} \\
(\mathrm{Yes} / \mathrm{No})\end{array}$} & \multirow{2}{*}{$\begin{array}{c}\text { Shot } \\
\text { Number }\end{array}$} & \multirow[t]{2}{*}{ Comments } \\
\hline$(\mathrm{ft} / \mathrm{s})$ & $(\mathrm{m} / \mathrm{s})$ & & & & & & \\
\hline 1941 & 592 & 0.25 & 0.00 & $\mathbf{C P}$ & Yes & 14356 & \\
\hline 1927 & 587 & 0.25 & -0.25 & CP & Yes & 14357 & \\
\hline 1885 & 575 & 0.00 & -0.50 & PP & Yes & 14358 & \\
\hline 1907 & 581 & 0.00 & 0.00 & PP & Yes & 14359 & \\
\hline & & & & & & & \\
\hline & & & & & & & \\
\hline & & & & & & & \\
\hline & & & & & & & \\
\hline & & & & & & & \\
\hline & & & & & & & \\
\hline & & & & & & & \\
\hline
\end{tabular}




\begin{tabular}{|c|c|c|c|c|c|c|c|}
\hline \multicolumn{2}{|c|}{ Target: } & \multicolumn{4}{|c|}{ 6055-T651 } & Date: & $4 / 4 / 2013$ \\
\hline \multicolumn{2}{|c|}{ Plate Number: } & \multicolumn{4}{|c|}{$521-881$} & Location: & EF 110G \\
\hline \multicolumn{2}{|c|}{ Thickness, in: } & \multicolumn{4}{|c|}{1.497} & & \\
\hline \multicolumn{2}{|c|}{ Thickness, mm: } & \multicolumn{4}{|l|}{38.02} & & \\
\hline \multicolumn{2}{|c|}{ Hardness, BHN: } & \multicolumn{4}{|l|}{121} & & \\
\hline \multicolumn{2}{|c|}{ Obliquity: } & \multicolumn{4}{|l|}{$0^{\circ}$} & & \\
\hline \multicolumn{2}{|c|}{ Projectile: } & \multicolumn{4}{|c|}{0.50 cal APM2 } & & \\
\hline \multicolumn{2}{|c|}{$\begin{array}{l}\text { Velocity } \\
\text { Measurement: }\end{array}$} & \multicolumn{4}{|l|}{ Xray } & & \\
\hline & $\mathrm{V}_{50}:$ & \multicolumn{2}{|c|}{$1917 \mathrm{ft} / \mathrm{s}$} & & \multicolumn{2}{|c|}{ Number of Shots: } & 4 \\
\hline & Std Dev: & \multicolumn{2}{|c|}{$29 \mathrm{ft} / \mathrm{s}$} & & \multicolumn{2}{|r|}{ Spread: } & $57 \mathrm{ft} / \mathrm{s}$ \\
\hline & ZMR: & \multicolumn{2}{|l|}{ N/A } & & & & \\
\hline \multicolumn{2}{|c|}{ Striking Velocity } & \multirow{2}{*}{$\begin{array}{l}\text { Pitch } \\
\text { (deg) }\end{array}$} & \multirow{2}{*}{$\begin{array}{l}\text { Yaw } \\
(\mathrm{deg})\end{array}$} & \multirow{2}{*}{$\begin{array}{l}\text { Result } \\
\text { (PP/CP) }\end{array}$} & \multirow{2}{*}{$\begin{array}{c}\text { Used for } \\
V_{50} \\
(\mathrm{Yes} / \mathrm{No})\end{array}$} & \multirow{2}{*}{$\begin{array}{c}\text { Shot } \\
\text { Number }\end{array}$} & \multirow[t]{2}{*}{ Comments } \\
\hline$(\mathrm{ft} / \mathrm{s})$ & $(\mathrm{m} / \mathrm{s})$ & & & & & & \\
\hline 1989 & 606 & -0.25 & -0.25 & $\mathrm{CP}$ & No & 14342 & \\
\hline 1888 & 575 & 0.00 & 0.00 & PP & Yes & 14343 & \\
\hline 1945 & 593 & 0.00 & 0.00 & $\mathbf{C P}$ & Yes & 14344 & \\
\hline 1939 & 591 & -0.25 & -0.25 & $\mathbf{C P}$ & Yes & 14345 & \\
\hline 1896 & 578 & 0.00 & 0.00 & $\mathbf{P P}$ & Yes & 14346 & \\
\hline & & & & & & & \\
\hline & & & & & & & \\
\hline & & & & & & & \\
\hline & & & & & & & \\
\hline & & & & & & & \\
\hline & & & & & & & \\
\hline
\end{tabular}




\begin{tabular}{|c|c|c|c|c|c|c|c|}
\hline \multirow{2}{*}{\multicolumn{2}{|c|}{$\begin{array}{l}\text { Target: } \\
\text { Plate Number: }\end{array}$}} & \multicolumn{4}{|c|}{ 6055-T651 } & Date: & $4 / 3 / 2013$ \\
\hline & & \multicolumn{4}{|c|}{$521-882$} & Location: & EF 110G \\
\hline \multicolumn{2}{|c|}{ Thickness, in: } & \multicolumn{4}{|c|}{1.499} & & \\
\hline \multicolumn{2}{|c|}{ Thickness, mm: } & \multicolumn{4}{|l|}{38.07} & & \\
\hline \multicolumn{2}{|c|}{ Hardness, BHN: } & \multicolumn{4}{|l|}{107} & & \\
\hline \multicolumn{2}{|c|}{ Obliquity: } & \multicolumn{4}{|l|}{$0^{\circ}$} & & \\
\hline \multicolumn{2}{|c|}{ Projectile: } & \multicolumn{4}{|c|}{0.50 cal APM2 } & & \\
\hline \multicolumn{2}{|c|}{$\begin{array}{l}\text { Velocity } \\
\text { Measurement: }\end{array}$} & \multicolumn{4}{|c|}{ Xray } & & \\
\hline \multicolumn{2}{|c|}{$\mathrm{V}_{50}:$} & \multicolumn{2}{|c|}{$1969 \mathrm{ft} / \mathrm{s}$} & & \multicolumn{2}{|c|}{ Number of Shots: } & 4 \\
\hline \multirow{2}{*}{\multicolumn{2}{|c|}{$\begin{array}{r}\text { Std Dev: } \\
\text { ZMR: }\end{array}$}} & \multicolumn{2}{|c|}{$28 \mathrm{ft} / \mathrm{s}$} & & \multicolumn{2}{|r|}{ Spread: } & $59 \mathrm{ft} / \mathrm{s}$ \\
\hline & & \multicolumn{2}{|l|}{ N/A } & & & & \\
\hline \multicolumn{2}{|c|}{ Striking Velocity } & \multirow{2}{*}{$\begin{array}{l}\text { Pitch } \\
\text { (deg) }\end{array}$} & \multirow{2}{*}{$\begin{array}{l}\text { Yaw } \\
(\mathrm{deg})\end{array}$} & \multirow{2}{*}{$\begin{array}{l}\text { Result } \\
(\mathrm{PP} / \mathrm{CP})\end{array}$} & \multirow{2}{*}{$\begin{array}{c}\text { Used for } \\
V_{50} \\
(\text { Yes/No) }\end{array}$} & \multirow{2}{*}{$\begin{array}{c}\text { Shot } \\
\text { Number }\end{array}$} & \multirow[t]{2}{*}{ Comments } \\
\hline$(\mathrm{ft} / \mathrm{s})$ & $(\mathrm{m} / \mathrm{s})$ & & & & & & \\
\hline 2271 & 692 & 0.00 & 0.75 & $\mathrm{CP}$ & No & 14333 & \\
\hline 1986 & 605 & 0.00 & 0.25 & $\mathbf{C P}$ & Yes & 14334 & \\
\hline 1882 & 574 & -0.25 & 0.00 & PP & No & 14335 & \\
\hline 1915 & 584 & 0.25 & -0.75 & PP & No & 14336 & \\
\hline 1914 & 583 & 0.25 & 0.00 & PP & No & 14337 & \\
\hline 1940 & 591 & -0.75 & 0.25 & PP & Yes & 14338 & \\
\hline 2021 & 616 & 0.00 & -0.25 & $\mathrm{CP}$ & No & 14339 & \\
\hline 1950 & 594 & 0.25 & 0.00 & $\mathbf{P P}$ & Yes & 14340 & \\
\hline 1999 & 609 & 0.00 & 0.00 & $\mathbf{C P}$ & Yes & 14341 & \\
\hline & & & & & & & \\
\hline & & & & & & & \\
\hline & & & & & & & \\
\hline
\end{tabular}




\begin{tabular}{|c|c|c|c|c|c|c|c|}
\hline \multicolumn{2}{|l|}{ Target: } & \multicolumn{4}{|c|}{ 6055-T651 } & Date: & $6 / 25 / 2013$ \\
\hline \multicolumn{2}{|c|}{ Plate Number: } & \multicolumn{4}{|c|}{$521-892$} & Location: & EF 108 \\
\hline \multicolumn{2}{|c|}{ Thickness, in: } & \multicolumn{4}{|l|}{1.498} & & \\
\hline \multicolumn{2}{|c|}{ Thickness, mm: } & \multicolumn{4}{|l|}{38.05} & & \\
\hline \multicolumn{2}{|c|}{ Hardness, BHN: } & \multicolumn{4}{|l|}{109} & & \\
\hline \multicolumn{2}{|c|}{ Obliquity: } & \multicolumn{4}{|l|}{$\mathbf{0}^{\circ}$} & & \\
\hline \multicolumn{2}{|c|}{ Projectile: } & \multicolumn{4}{|c|}{0.50 cal APM2 } & & \\
\hline \multicolumn{2}{|c|}{$\begin{array}{l}\text { Velocity } \\
\text { Measurement: }\end{array}$} & \multicolumn{4}{|c|}{ Xray } & & \\
\hline \multicolumn{2}{|c|}{$\mathrm{V}_{50}:$} & \multicolumn{2}{|c|}{$1977 \mathrm{ft} / \mathrm{s}$} & & \multicolumn{2}{|c|}{ Number of Shots: } & 6 \\
\hline & Std Dev: & $36 \mathrm{ft} / \mathrm{t}$ & & & & Spread: & $87 \mathrm{ft} / \mathrm{s}$ \\
\hline \multicolumn{2}{|c|}{ ZMR: } & \multicolumn{2}{|c|}{$12 \mathrm{ft} / \mathrm{s}$} & & & & \\
\hline \multicolumn{2}{|c|}{ Striking Velocity } & \multirow{2}{*}{$\begin{array}{l}\text { Pitch } \\
\text { (deg) }\end{array}$} & \multirow{2}{*}{$\begin{array}{l}\text { Yaw } \\
(\mathrm{deg})\end{array}$} & \multirow{2}{*}{$\begin{array}{c}\text { Result } \\
(\mathrm{PP} / \mathrm{CP})\end{array}$} & \multirow{2}{*}{$\begin{array}{c}\text { Used for } \\
\mathrm{V}_{50} \\
(\mathrm{Yes} / \mathrm{No}) \\
\end{array}$} & \multirow{2}{*}{$\begin{array}{c}\text { Shot } \\
\text { Number }\end{array}$} & \multirow[t]{2}{*}{ Comments } \\
\hline$(\mathrm{ft} / \mathrm{s})$ & $(\mathrm{m} / \mathrm{s})$ & & & & & & \\
\hline 1742 & 531 & -- & -- & $\mathrm{PP}$ & No & 11437 & \\
\hline 1956 & 596 & -- & -- & $\mathbf{C P}$ & Yes & 11438 & \\
\hline 1851 & 564 & -- & -- & $\mathrm{PP}$ & No & 11439 & \\
\hline 1928 & 588 & -- & -- & $\mathrm{PP}$ & No & 11440 & \\
\hline 1945 & 593 & -- & -- & PP & Yes & 11441 & \\
\hline 1947 & 593 & -- & -- & PP & Yes & 11442 & \\
\hline 1674 & 510 & -- & -- & PP & No & 11443 & \\
\hline 2011 & 613 & -- & -- & CP & Yes & 11444 & \\
\hline 1968 & 600 & -- & -- & PP & Yes & 11445 & \\
\hline 1526 & 465 & -- & -- & $\mathrm{PP}$ & No & 11446 & \\
\hline 1484 & 452 & -- & -- & $\mathrm{PP}$ & No & 11447 & \\
\hline 1611 & 491 & -- & -- & $\mathrm{PP}$ & No & 11448 & \\
\hline 1679 & 512 & -- & -- & $\mathrm{PP}$ & No & 11449 & \\
\hline 1903 & 580 & -- & -- & PP & No & 11450 & \\
\hline 2032 & 619 & -- & -- & $\mathbf{C P}$ & Yes & 11451 & \\
\hline
\end{tabular}




\begin{tabular}{|c|c|c|c|c|c|c|c|}
\hline \multicolumn{2}{|c|}{ Target: } & \multicolumn{4}{|c|}{ 6055-T651 } & Date: & 4/12/2013 \\
\hline \multicolumn{2}{|c|}{ Plate Number: } & \multicolumn{4}{|c|}{$521-901$} & Location: & EF 110G \\
\hline \multicolumn{2}{|c|}{ Thickness, in: } & \multicolumn{4}{|c|}{1.977} & & \\
\hline \multicolumn{2}{|c|}{ Thickness, mm: } & \multicolumn{4}{|l|}{50.22} & & \\
\hline \multicolumn{2}{|c|}{ Hardness, BHN: } & \multicolumn{4}{|l|}{101} & & \\
\hline \multicolumn{2}{|c|}{ Obliquity: } & \multicolumn{4}{|l|}{$0^{\circ}$} & & \\
\hline \multicolumn{2}{|c|}{ Projectile: } & \multicolumn{4}{|c|}{0.50 cal APM2 } & & \\
\hline \multicolumn{2}{|c|}{$\begin{array}{l}\text { Velocity } \\
\text { Measurement: }\end{array}$} & \multicolumn{4}{|l|}{ Xray } & & \\
\hline \multicolumn{2}{|r|}{$\mathrm{V}_{50}:$} & \multicolumn{2}{|c|}{$2254 \mathrm{ft} / \mathrm{s}$} & & \multicolumn{2}{|c|}{ Number of Shots: } & 4 \\
\hline & Std Dev: & \multicolumn{2}{|c|}{$15 \mathrm{ft} / \mathrm{s}$} & & \multicolumn{2}{|r|}{ Spread: } & $33 \mathrm{ft} / \mathrm{s}$ \\
\hline & ZMR: & \multicolumn{2}{|l|}{ N/A } & & & & \\
\hline \multicolumn{2}{|c|}{ Striking Velocity } & \multirow{2}{*}{$\begin{array}{l}\text { Pitch } \\
\text { (deg) }\end{array}$} & \multirow{2}{*}{$\begin{array}{l}\text { Yaw } \\
(\mathrm{deg})\end{array}$} & \multirow{2}{*}{$\begin{array}{l}\text { Result } \\
\text { (PP/CP) }\end{array}$} & \multirow{2}{*}{$\begin{array}{c}\text { Used for } \\
V_{50} \\
(\text { Yes/No) }\end{array}$} & \multirow{2}{*}{$\begin{array}{c}\text { Shot } \\
\text { Number }\end{array}$} & \multirow[t]{2}{*}{ Comments } \\
\hline$(\mathrm{ft} / \mathrm{s})$ & $(\mathrm{m} / \mathrm{s})$ & & & & & & \\
\hline 2343 & 714 & 0.25 & -0.50 & $\mathrm{CP}$ & No & 14373 & \\
\hline 2313 & 705 & 0.25 & 0.00 & $\mathrm{CP}$ & No & 14374 & \\
\hline 2260 & 689 & -1.00 & 0.25 & $\mathbf{C P}$ & Yes & 14375 & \\
\hline 2212 & 674 & -0.25 & 0.00 & $\mathrm{PP}$ & No & 14376 & \\
\hline 2239 & 682 & 0.00 & 0.75 & PP & Yes & 14377 & \\
\hline 2243 & 684 & 0.00 & 0.00 & PP & Yes & 14378 & \\
\hline 2272 & 692 & 0.25 & -0.25 & $\mathbf{C P}$ & Yes & 14379 & \\
\hline & & & & & & & \\
\hline & & & & & & & \\
\hline & & & & & & & \\
\hline & & & & & & & \\
\hline & & & & & & & \\
\hline
\end{tabular}




\begin{tabular}{|c|c|c|c|c|c|c|c|}
\hline \multicolumn{2}{|l|}{ Target: } & \multicolumn{4}{|c|}{ 6055-T651 } & Date: & $6 / 19 / 2013$ \\
\hline \multicolumn{2}{|c|}{ Plate Number: } & \multicolumn{4}{|c|}{$521-902$} & Location: & EF 108 \\
\hline \multicolumn{2}{|c|}{ Thickness, in: } & \multicolumn{4}{|l|}{1.790} & & \\
\hline \multicolumn{2}{|c|}{ Thickness, mm: } & \multicolumn{4}{|l|}{45.47} & & \\
\hline \multicolumn{2}{|c|}{ Hardness, BHN: } & \multicolumn{4}{|l|}{112} & & \\
\hline \multicolumn{2}{|c|}{ Obliquity: } & \multicolumn{4}{|l|}{$0^{\circ}$} & & \\
\hline \multicolumn{2}{|c|}{ Projectile: } & \multicolumn{4}{|c|}{0.50 cal APM2 } & & \\
\hline \multicolumn{2}{|c|}{\begin{tabular}{|l|} 
Velocity \\
Measurement:
\end{tabular}} & \multicolumn{4}{|c|}{ Xray } & & \\
\hline \multicolumn{2}{|r|}{$\mathrm{V}_{50}$ : } & \multicolumn{2}{|c|}{$2251 \mathrm{ft} / \mathrm{s}$} & & \multicolumn{2}{|c|}{ Number of Shots: } & 4 \\
\hline & Std Dev: & \multicolumn{2}{|c|}{$29 \mathrm{ft} / \mathrm{s}$} & & \multicolumn{2}{|c|}{ Spread: } & $58 \mathrm{ft} / \mathrm{s}$ \\
\hline & ZMR: & \multicolumn{2}{|l|}{ N/A } & & & & \\
\hline \multicolumn{2}{|c|}{ Striking Velocity } & \multirow{2}{*}{$\begin{array}{l}\text { Pitch } \\
\text { (deg) }\end{array}$} & \multirow{2}{*}{$\begin{array}{l}\text { Yaw } \\
(\mathrm{deg})\end{array}$} & \multirow{2}{*}{$\begin{array}{l}\text { Result } \\
\text { (PP/CP) }\end{array}$} & \multirow{2}{*}{$\begin{array}{c}\text { Used for } \\
\mathrm{V}_{50} \\
(\mathrm{Yes} / \mathrm{No})\end{array}$} & \multirow{2}{*}{$\begin{array}{c}\text { Shot } \\
\text { Number }\end{array}$} & \multirow[t]{2}{*}{ Comments } \\
\hline$(\mathrm{ft} / \mathrm{s})$ & $(\mathrm{m} / \mathrm{s})$ & & & & & & \\
\hline 2296 & 700 & -- & -- & $\mathrm{CP}$ & No & 11431 & \\
\hline 2277 & 694 & -- & -- & CP & Yes & 11432 & \\
\hline 2151 & 656 & -- & -- & $\mathrm{PP}$ & No & 11433 & \\
\hline 2219 & 676 & -- & -- & PP & Yes & 11434 & \\
\hline 2275 & 693 & -- & -- & $\mathbf{C P}$ & Yes & 11435 & \\
\hline 2233 & 681 & -- & -- & PP & Yes & 11436 & \\
\hline & & & & & & & \\
\hline & & & & & & & \\
\hline & & & & & & & \\
\hline & & & & & & & \\
\hline & & & & & & & \\
\hline & & & & & & & \\
\hline
\end{tabular}




\begin{tabular}{|c|c|c|c|c|c|c|c|}
\hline \multicolumn{2}{|c|}{ Target: } & \multicolumn{4}{|c|}{ 6055-T651 } & Date: & 4/11/2013 \\
\hline \multicolumn{2}{|c|}{ Plate Number: } & \multicolumn{4}{|c|}{$521-911$} & Location: & EF 110G \\
\hline \multicolumn{2}{|c|}{ Thickness, in: } & \multicolumn{4}{|c|}{1.999} & & \\
\hline \multicolumn{2}{|c|}{ Thickness, mm: } & \multicolumn{4}{|l|}{50.77} & & \\
\hline \multicolumn{2}{|c|}{ Hardness, BHN: } & \multicolumn{4}{|l|}{107} & & \\
\hline \multicolumn{2}{|c|}{ Obliquity: } & \multicolumn{4}{|l|}{$0^{\circ}$} & & \\
\hline \multicolumn{2}{|c|}{ Projectile: } & \multicolumn{4}{|c|}{0.50 cal APM2 } & & \\
\hline \multicolumn{2}{|c|}{$\begin{array}{l}\text { Velocity } \\
\text { Measurement: }\end{array}$} & \multicolumn{4}{|l|}{ Xray } & & \\
\hline \multicolumn{2}{|c|}{$\mathrm{V}_{50}:$} & \multicolumn{2}{|c|}{$2309 \mathrm{ft} / \mathrm{s}$} & & \multicolumn{2}{|c|}{ Number of Shots: } & 4 \\
\hline & Std Dev & \multicolumn{2}{|c|}{$16 \mathrm{ft} / \mathrm{s}$} & & \multicolumn{2}{|r|}{ Spread: } & $39 \mathrm{ft} / \mathrm{s}$ \\
\hline & ZMR & \multicolumn{2}{|l|}{ N/A } & & & & \\
\hline \multicolumn{2}{|c|}{ Striking Velocity } & \multirow{2}{*}{$\begin{array}{l}\text { Pitch } \\
\text { (deg) }\end{array}$} & \multirow{2}{*}{$\begin{array}{l}\text { Yaw } \\
(\mathrm{deg})\end{array}$} & \multirow{2}{*}{$\begin{array}{l}\text { Result } \\
\text { (PP/CP) }\end{array}$} & \multirow{2}{*}{$\begin{array}{c}\text { Used for } \\
V_{50} \\
(\text { Yes/No) }\end{array}$} & \multirow{2}{*}{$\begin{array}{c}\text { Shot } \\
\text { Number }\end{array}$} & \multirow[t]{2}{*}{ Comments } \\
\hline$(\mathrm{ft} / \mathrm{s})$ & $(\mathrm{m} / \mathrm{s})$ & & & & & & \\
\hline 2289 & 698 & 0.25 & 0.25 & $\mathbf{P P}$ & Yes & 14368 & \\
\hline 2287 & 697 & 0.25 & 0.00 & PP & No & 14369 & \\
\hline 2312 & 705 & 0.25 & -0.25 & $\mathbf{C P}$ & Yes & 14370 & \\
\hline 2305 & 703 & 0.25 & 0.00 & $\mathbf{P P}$ & Yes & 14371 & \\
\hline 2328 & 710 & 0.25 & 0.00 & $\mathbf{C P}$ & Yes & 14372 & \\
\hline & & & & & & & \\
\hline & & & & & & & \\
\hline & & & & & & & \\
\hline & & & & & & & \\
\hline & & & & & & & \\
\hline & & & & & & & \\
\hline
\end{tabular}




\begin{tabular}{|c|c|c|c|c|c|c|c|}
\hline \multicolumn{2}{|l|}{ Target: } & \multicolumn{4}{|c|}{ 6055-T651 } & Date: & $4 / 11 / 2013$ \\
\hline \multicolumn{2}{|c|}{ Plate Number: } & \multicolumn{4}{|c|}{$521-921$} & Location: & EF 110G \\
\hline \multicolumn{2}{|c|}{ Thickness, in: } & \multicolumn{4}{|c|}{2.004} & & \\
\hline \multicolumn{2}{|c|}{ Thickness, mm: } & \multicolumn{4}{|l|}{50.90} & & \\
\hline \multicolumn{2}{|c|}{ Hardness, BHN: } & \multicolumn{4}{|l|}{107} & & \\
\hline \multicolumn{2}{|c|}{ Obliquity: } & \multicolumn{4}{|l|}{$0^{\circ}$} & & \\
\hline \multicolumn{2}{|c|}{ Projectile: } & \multicolumn{4}{|c|}{0.50 cal APM2 } & & \\
\hline \multicolumn{2}{|c|}{$\begin{array}{l}\text { Velocity } \\
\text { Measurement: }\end{array}$} & \multicolumn{4}{|l|}{ Xray } & & \\
\hline \multicolumn{2}{|c|}{$\mathrm{V}_{50}:$} & \multicolumn{2}{|c|}{$2286 \mathrm{ft} / \mathrm{s}$} & & \multicolumn{2}{|c|}{ Number of Shots: } & 4 \\
\hline & Std Dev: & \multicolumn{2}{|c|}{$19 \mathrm{ft} / \mathrm{s}$} & & \multicolumn{2}{|r|}{ Spread: } & $41 \mathrm{ft} / \mathrm{s}$ \\
\hline & ZMR: & \multicolumn{2}{|c|}{$28 \mathrm{ft} / \mathrm{s}$} & & & & \\
\hline \multicolumn{2}{|c|}{ Striking Velocity } & \multirow{2}{*}{$\begin{array}{l}\text { Pitch } \\
\text { (deg) }\end{array}$} & \multirow{2}{*}{$\begin{array}{l}\text { Yaw } \\
(\mathrm{deg})\end{array}$} & \multirow{2}{*}{$\begin{array}{l}\text { Result } \\
\text { (PP/CP) }\end{array}$} & \multirow{2}{*}{$\begin{array}{c}\text { Used for } \\
V_{50} \\
(\mathrm{Yes} / \mathrm{No})\end{array}$} & \multirow{2}{*}{$\begin{array}{c}\text { Shot } \\
\text { Number }\end{array}$} & \multirow[t]{2}{*}{ Comments } \\
\hline$(\mathrm{ft} / \mathrm{s})$ & $(\mathrm{m} / \mathrm{s})$ & & & & & & \\
\hline 2295 & 699 & 0.00 & -0.25 & PP & Yes & 14360 & \\
\hline 2350 & 716 & -0.50 & 0.00 & $\mathrm{CP}$ & No & 14361 & \\
\hline 2335 & 712 & 0.00 & 0.00 & $\mathrm{CP}$ & No & 14362 & \\
\hline 2308 & 703 & 0.25 & 0.00 & $\mathbf{C P}$ & Yes & 14363 & \\
\hline 2314 & 705 & 0.00 & 0.00 & $\mathrm{CP}$ & No & 14364 & \\
\hline 2267 & 691 & 0.00 & -0.25 & $\mathbf{C P}$ & Yes & 14365 & \\
\hline 2228 & 679 & 0.00 & 0.00 & $\mathrm{PP}$ & No & 14366 & \\
\hline 2273 & 693 & 0.00 & 0.00 & $\mathbf{P P}$ & Yes & 14367 & \\
\hline & & & & & & & \\
\hline & & & & & & & \\
\hline & & & & & & & \\
\hline & & & & & & & \\
\hline
\end{tabular}




\begin{tabular}{|c|c|c|c|c|c|c|c|}
\hline \multirow{2}{*}{\multicolumn{2}{|c|}{$\begin{array}{l}\text { Target: } \\
\text { Plate Number: }\end{array}$}} & \multicolumn{4}{|c|}{ 6055-T651 } & Date: & $6 / 12 / 2013$ \\
\hline & & 521-92 & & & & Location: & EF 108 \\
\hline \multicolumn{2}{|c|}{ Thickness, in: } & \multicolumn{4}{|c|}{1.980} & & \\
\hline \multicolumn{2}{|c|}{ Thickness, mm: } & \multicolumn{4}{|l|}{50.29} & & \\
\hline \multicolumn{2}{|c|}{ Hardness, BHN: } & \multicolumn{4}{|l|}{116} & & \\
\hline \multicolumn{2}{|c|}{ Obliquity: } & \multicolumn{4}{|l|}{$\mathbf{0}^{\circ}$} & & \\
\hline \multicolumn{2}{|c|}{ Projectile: } & \multicolumn{4}{|c|}{0.50 cal APM2 } & & \\
\hline \multicolumn{2}{|c|}{$\begin{array}{l}\text { Velocity } \\
\text { Measurement: }\end{array}$} & \multicolumn{4}{|c|}{ Xray } & & \\
\hline \multicolumn{2}{|c|}{$\mathrm{V}_{50}:$} & \multicolumn{2}{|c|}{$2278 \mathrm{ft} / \mathrm{s}$} & & \multicolumn{2}{|c|}{ Number of Shots: } & 4 \\
\hline & Std Dev: & \multicolumn{2}{|c|}{$21 \mathrm{ft} / \mathrm{s}$} & & \multicolumn{2}{|c|}{ Spread: } & $49 \mathrm{ft} / \mathrm{s}$ \\
\hline & ZMR: & \multicolumn{2}{|l|}{ N/A } & & & & \\
\hline \multicolumn{2}{|c|}{ Striking Velocity } & \multirow{2}{*}{$\begin{array}{l}\text { Pitch } \\
(\mathrm{deg})\end{array}$} & \multirow{2}{*}{$\begin{array}{l}\text { Yaw } \\
(\mathrm{deg})\end{array}$} & \multirow{2}{*}{$\begin{array}{l}\text { Result } \\
(\mathrm{PP} / \mathrm{CP})\end{array}$} & \multirow{2}{*}{$\begin{array}{c}\text { Used for } \\
\mathrm{V}_{50} \\
(\mathrm{Yes} / \mathrm{No})\end{array}$} & \multirow{2}{*}{$\begin{array}{c}\text { Shot } \\
\text { Number }\end{array}$} & \multirow[t]{2}{*}{ Comments } \\
\hline$(\mathrm{ft} / \mathrm{s})$ & $(\mathrm{m} / \mathrm{s})$ & & & & & & \\
\hline 2325 & 709 & -- & -- & $\mathrm{CP}$ & No & 11423 & \\
\hline 2093 & 638 & -- & -- & PP & No & 11424 & \\
\hline 2168 & 661 & -- & -- & PP & No & 11425 & \\
\hline 2253 & 687 & -- & -- & $\mathbf{P P}$ & Yes & 11426 & \\
\hline 2338 & 713 & -- & -- & $\mathrm{CP}$ & No & 11427 & \\
\hline 2302 & 702 & -- & -- & CP & Yes & 11428 & \\
\hline 2272 & 692 & -- & -- & PP & Yes & 11429 & \\
\hline 2285 & 696 & -- & -- & $\mathbf{C P}$ & Yes & 11430 & \\
\hline & & & & & & & \\
\hline & & & & & & & \\
\hline & & & & & & & \\
\hline & & & & & & & \\
\hline
\end{tabular}




\begin{tabular}{|c|c|c|c|c|c|c|c|}
\hline \multicolumn{2}{|c|}{ Target: } & \multicolumn{4}{|c|}{ 6055-T651 } & Date: & 4/16/2013 \\
\hline \multicolumn{2}{|c|}{ Plate Number: } & \multicolumn{4}{|c|}{$521-941$} & Location: & EF 110G \\
\hline \multicolumn{2}{|c|}{ Thickness, in: } & \multicolumn{4}{|c|}{2.500} & & \\
\hline \multicolumn{2}{|c|}{ Thickness, mm: } & \multicolumn{4}{|l|}{63.50} & & \\
\hline \multicolumn{2}{|c|}{ Hardness, BHN: } & \multicolumn{4}{|l|}{112} & & \\
\hline \multicolumn{2}{|c|}{ Obliquity: } & \multicolumn{4}{|l|}{$0^{\circ}$} & & \\
\hline \multicolumn{2}{|c|}{ Projectile: } & \multicolumn{4}{|c|}{0.50 cal APM2 } & & \\
\hline \multicolumn{2}{|c|}{$\begin{array}{l}\text { Velocity } \\
\text { Measurement: }\end{array}$} & \multicolumn{4}{|l|}{ Xray } & & \\
\hline & $\mathrm{V}_{50}:$ & \multicolumn{2}{|c|}{$2615 \mathrm{ft} / \mathrm{s}$} & & \multicolumn{2}{|c|}{ Number of Shots: } & 4 \\
\hline & Std Dev: & \multicolumn{2}{|c|}{$24 \mathrm{ft} / \mathrm{s}$} & & \multicolumn{2}{|r|}{ Spread: } & $57 \mathrm{ft} / \mathrm{s}$ \\
\hline & ZMR: & \multicolumn{2}{|l|}{ N/A } & & & & \\
\hline \multicolumn{2}{|c|}{ Striking Velocity } & \multirow{2}{*}{$\begin{array}{l}\text { Pitch } \\
\text { (deg) }\end{array}$} & \multirow{2}{*}{$\begin{array}{l}\text { Yaw } \\
(\mathrm{deg})\end{array}$} & \multirow{2}{*}{$\begin{array}{l}\text { Result } \\
(\mathrm{PP} / \mathrm{CP})\end{array}$} & \multirow{2}{*}{$\begin{array}{c}\text { Used for } \\
V_{50} \\
(\text { Yes/No) }\end{array}$} & \multirow{2}{*}{$\begin{array}{c}\text { Shot } \\
\text { Number }\end{array}$} & \multirow[t]{2}{*}{ Comments } \\
\hline$(\mathrm{ft} / \mathrm{s})$ & $(\mathrm{m} / \mathrm{s})$ & & & & & & \\
\hline 2660 & 811 & 0.00 & 1.00 & $\mathrm{CP}$ & No & 14387 & \\
\hline 2616 & 797 & 0.25 & 0.00 & PP & Yes & 14388 & \\
\hline 2667 & 813 & 0.50 & 0.25 & $\mathrm{CP}$ & No & 14389 & \\
\hline 2583 & 787 & 0.25 & 0.00 & $\mathbf{P P}$ & Yes & 14390 & \\
\hline 2620 & 799 & 0.00 & -0.25 & $\mathbf{C P}$ & Yes & 14391 & \\
\hline 2640 & 805 & 0.50 & 0.25 & $\mathbf{C P}$ & Yes & 14392 & \\
\hline & & & & & & & \\
\hline & & & & & & & \\
\hline & & & & & & & \\
\hline & & & & & & & \\
\hline & & & & & & & \\
\hline & & & & & & & \\
\hline
\end{tabular}




\begin{tabular}{|c|c|c|c|c|c|c|c|}
\hline \multicolumn{2}{|l|}{ Target: } & \multicolumn{4}{|c|}{ 6055-T651 } & Date: & $4 / 15 / 2013$ \\
\hline \multicolumn{2}{|c|}{ Plate Number: } & \multicolumn{4}{|c|}{$521-942$} & Location: & EF 110G \\
\hline \multicolumn{2}{|c|}{ Thickness, in: } & \multicolumn{4}{|c|}{2.487} & & \\
\hline \multicolumn{2}{|c|}{ Thickness, mm: } & \multicolumn{4}{|l|}{63.17} & & \\
\hline \multicolumn{2}{|c|}{ Hardness, BHN: } & \multicolumn{4}{|l|}{112} & & \\
\hline \multicolumn{2}{|c|}{ Obliquity: } & \multicolumn{4}{|l|}{$0^{\circ}$} & & \\
\hline \multicolumn{2}{|c|}{ Projectile: } & \multicolumn{4}{|c|}{0.50 cal APM2 } & & \\
\hline \multicolumn{2}{|c|}{$\begin{array}{l}\text { Velocity } \\
\text { Measurement: }\end{array}$} & \multicolumn{4}{|l|}{ Xray } & & \\
\hline \multicolumn{2}{|c|}{$\mathrm{V}_{50}$ : } & \multicolumn{2}{|c|}{$2671 \mathrm{ft} / \mathrm{s}$} & & \multicolumn{2}{|c|}{ Number of Shots: } & 4 \\
\hline & Std Dev & \multicolumn{2}{|c|}{$27 \mathrm{ft} / \mathrm{s}$} & & \multicolumn{2}{|r|}{ Spread: } & $58 \mathrm{ft} / \mathrm{s}$ \\
\hline & ZMR & \multicolumn{2}{|l|}{ N/A } & & & & \\
\hline \multicolumn{2}{|c|}{ Striking Velocity } & \multirow{2}{*}{$\begin{array}{l}\text { Pitch } \\
\text { (deg) }\end{array}$} & \multirow{2}{*}{$\begin{array}{l}\text { Yaw } \\
(\mathrm{deg})\end{array}$} & \multirow{2}{*}{$\begin{array}{l}\text { Result } \\
\text { (PP/CP) }\end{array}$} & \multirow{2}{*}{$\begin{array}{c}\text { Used for } \\
V_{50} \\
(\text { Yes/No) }\end{array}$} & \multirow{2}{*}{$\begin{array}{c}\text { Shot } \\
\text { Number }\end{array}$} & \multirow[t]{2}{*}{ Comments } \\
\hline$(\mathrm{ft} / \mathrm{s})$ & $(\mathrm{m} / \mathrm{s})$ & & & & & & \\
\hline 2622 & 799 & 0.00 & 0.25 & PP & No & 14380 & \\
\hline 2758 & 841 & 0.00 & -0.50 & $\mathrm{CP}$ & No & 14381 & \\
\hline 2706 & 825 & 1.50 & -0.25 & $\mathbf{C P}$ & Yes & 14382 & \\
\hline 2648 & 807 & 0.25 & 0.50 & $\mathbf{P P}$ & Yes & 14383 & \\
\hline 2678 & 816 & 0.00 & 0.00 & $\mathbf{C P}$ & Yes & 14384 & \\
\hline 2662 & 811 & 0.00 & 0.00 & $\mathrm{X}$ & No & 14385 & Projectile Hit Clamp - No Test \\
\hline 2651 & 808 & 0.25 & 0.75 & PP & Yes & 14386 & \\
\hline & & & & & & & \\
\hline & & & & & & & \\
\hline & & & & & & & \\
\hline & & & & & & & \\
\hline & & & & & & & \\
\hline
\end{tabular}




\begin{tabular}{|c|c|c|c|c|c|c|c|}
\hline \multicolumn{2}{|l|}{ Target: } & \multicolumn{4}{|c|}{ 6055-T651 } & Date: & $6 / 11 / 2013$ \\
\hline \multicolumn{2}{|c|}{ Plate Number: } & \multicolumn{4}{|c|}{ 521-951 } & Location: & EF 108 \\
\hline \multicolumn{2}{|c|}{ Thickness, in: } & \multicolumn{4}{|l|}{2.918} & & \\
\hline \multicolumn{2}{|c|}{ Thickness, mm: } & \multicolumn{4}{|l|}{74.12} & & \\
\hline \multicolumn{2}{|c|}{ Hardness, BHN: } & \multicolumn{4}{|l|}{124} & & \\
\hline \multicolumn{2}{|c|}{ Obliquity: } & \multicolumn{4}{|l|}{$0^{\circ}$} & & \\
\hline \multicolumn{2}{|c|}{ Projectile: } & \multicolumn{4}{|c|}{0.50 cal APM2 } & & \\
\hline \multicolumn{2}{|c|}{\begin{tabular}{|l|} 
Velocity \\
Measurement:
\end{tabular}} & \multicolumn{4}{|c|}{ Xray } & & \\
\hline \multicolumn{2}{|r|}{$\mathrm{V}_{50}$ : } & \multicolumn{2}{|c|}{$2974 \mathrm{ft} / \mathrm{s}$} & & \multicolumn{2}{|c|}{ Number of Shots: } & 4 \\
\hline & Std Dev: & \multicolumn{2}{|c|}{$25 \mathrm{ft} / \mathrm{s}$} & & \multicolumn{2}{|c|}{ Spread: } & $57 \mathrm{ft} / \mathrm{s}$ \\
\hline & ZMR: & \multicolumn{2}{|l|}{ N/A } & & & & \\
\hline \multicolumn{2}{|c|}{ Striking Velocity } & \multirow{2}{*}{$\begin{array}{l}\text { Pitch } \\
\text { (deg) }\end{array}$} & \multirow{2}{*}{$\begin{array}{l}\text { Yaw } \\
(\mathrm{deg})\end{array}$} & \multirow{2}{*}{$\begin{array}{c}\text { Result } \\
\text { (PP/CP) }\end{array}$} & \multirow{2}{*}{$\begin{array}{c}\text { Used for } \\
\mathrm{V}_{50} \\
(\text { Yes/No) }\end{array}$} & \multirow{2}{*}{$\begin{array}{c}\text { Shot } \\
\text { Number }\end{array}$} & \multirow[t]{2}{*}{ Comments } \\
\hline$(\mathrm{ft} / \mathrm{s})$ & $(\mathrm{m} / \mathrm{s})$ & & & & & & \\
\hline 2893 & 882 & -- & -- & PP & No & 11418 & \\
\hline 3009 & 917 & -- & -- & CP & Yes & 11419 & \\
\hline 2959 & 902 & -- & -- & PP & Yes & 11420 & \\
\hline 2975 & 907 & -- & -- & $\mathbf{C P}$ & Yes & 11421 & \\
\hline 2952 & 900 & -- & -- & PP & Yes & 11422 & \\
\hline & & & & & & & \\
\hline & & & & & & & \\
\hline & & & & & & & \\
\hline & & & & & & & \\
\hline & & & & & & & \\
\hline & & & & & & & \\
\hline & & & & & & & \\
\hline
\end{tabular}




\begin{tabular}{|c|c|c|c|c|c|c|c|}
\hline \multicolumn{2}{|l|}{ Target: } & \multicolumn{4}{|c|}{ 6055-T651 } & Date: & 6/6/2013 \\
\hline \multicolumn{2}{|c|}{ Plate Number: } & \multicolumn{4}{|c|}{$521-951$} & Location: & EF 108 \\
\hline \multicolumn{2}{|c|}{ Thickness, in: } & \multicolumn{4}{|c|}{2.918} & & \\
\hline \multicolumn{2}{|c|}{ Thickness, mm: } & \multicolumn{4}{|l|}{74.12} & & \\
\hline \multicolumn{2}{|c|}{ Hardness, BHN: } & \multicolumn{4}{|l|}{124} & & \\
\hline \multicolumn{2}{|c|}{ Obliquity: } & \multicolumn{4}{|l|}{$0^{\circ}$} & & \\
\hline \multicolumn{2}{|c|}{ Projectile: } & \multicolumn{4}{|c|}{$14.5 \mathrm{~mm}$ BS41 } & & \\
\hline \multicolumn{2}{|c|}{$\begin{array}{l}\text { Velocity } \\
\text { Measurement: }\end{array}$} & \multicolumn{4}{|l|}{ Xray } & & \\
\hline \multicolumn{2}{|r|}{$\mathrm{V}_{50}:$} & \multicolumn{2}{|c|}{$2752 \mathrm{ft} / \mathrm{s}$} & & \multicolumn{2}{|c|}{ Number of Shots: } & 6 \\
\hline & Std Dev: & \multicolumn{2}{|c|}{$32 \mathrm{ft} / \mathrm{s}$} & & \multicolumn{2}{|r|}{ Spread: } & $84 \mathrm{ft} / \mathrm{s}$ \\
\hline & ZMR: & \multicolumn{2}{|l|}{ N/A } & & & & \\
\hline \multicolumn{2}{|c|}{ Striking Velocity } & \multirow{2}{*}{$\begin{array}{l}\text { Pitch } \\
\text { (deg) }\end{array}$} & \multirow{2}{*}{$\begin{array}{l}\text { Yaw } \\
(\mathrm{deg})\end{array}$} & \multirow{2}{*}{$\begin{array}{l}\text { Result } \\
\text { (PP/CP) }\end{array}$} & \multirow{2}{*}{$\begin{array}{c}\text { Used for } \\
V_{50} \\
(\text { Yes/No) }\end{array}$} & \multirow{2}{*}{$\begin{array}{c}\text { Shot } \\
\text { Number }\end{array}$} & \multirow[t]{2}{*}{ Comments } \\
\hline$(\mathrm{ft} / \mathrm{s})$ & $(\mathrm{m} / \mathrm{s})$ & & & & & & \\
\hline 2508 & 764 & -- & -- & PP & No & 11410 & \\
\hline 2604 & 794 & -- & -- & PP & No & 11411 & \\
\hline 2793 & 851 & -- & -- & $\mathbf{C P}$ & Yes & 11412 & \\
\hline 2709 & 826 & -- & -- & $\mathbf{P P}$ & Yes & 11413 & \\
\hline 2722 & 830 & -- & -- & PP & Yes & 11414 & \\
\hline 2757 & 840 & -- & -- & CP & Yes & 11415 & \\
\hline 2752 & 839 & -- & -- & PP & Yes & 11416 & \\
\hline 2779 & 847 & -- & -- & $\mathbf{C P}$ & Yes & 11417 & \\
\hline & & & & & & & \\
\hline & & & & & & & \\
\hline & & & & & & & \\
\hline & & & & & & & \\
\hline
\end{tabular}


$\underline{0.50-\mathrm{cal} \text { FSP }}$

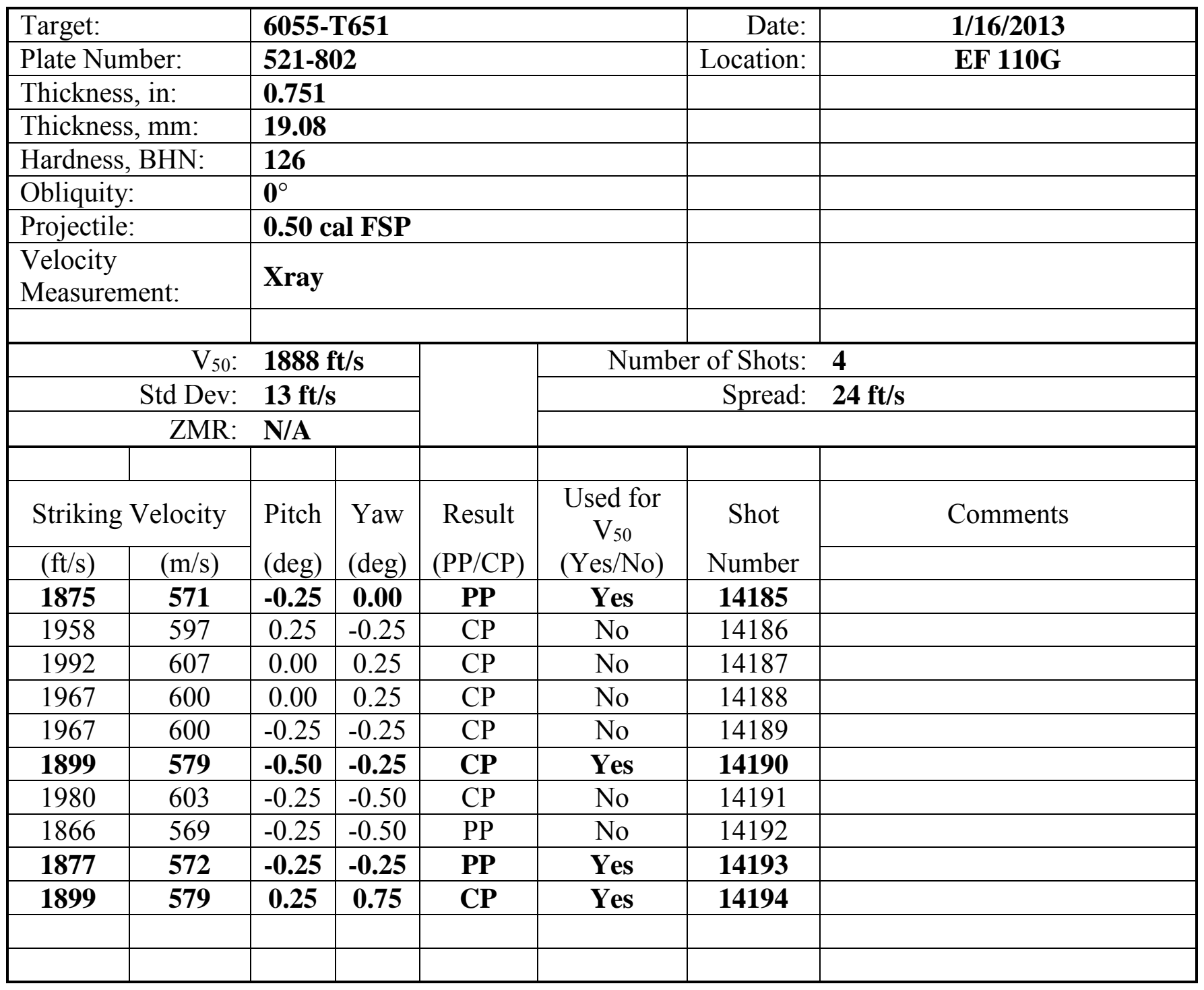




\begin{tabular}{|c|c|c|c|c|c|c|c|}
\hline \multicolumn{2}{|l|}{ Target: } & \multicolumn{4}{|c|}{ 6055-T651 } & Date: & $1 / 15 / 2013$ \\
\hline \multicolumn{2}{|c|}{ Plate Number: } & \multicolumn{4}{|c|}{$521-812$} & Location: & EF 110G \\
\hline \multicolumn{2}{|c|}{ Thickness, in: } & \multicolumn{4}{|l|}{0.753} & & \\
\hline \multicolumn{2}{|c|}{ Thickness, mm: } & \multicolumn{4}{|l|}{19.13} & & \\
\hline \multicolumn{2}{|c|}{ Hardness, BHN: } & \multicolumn{4}{|l|}{126} & & \\
\hline \multicolumn{2}{|c|}{ Obliquity: } & \multicolumn{4}{|l|}{$0^{\circ}$} & & \\
\hline \multicolumn{2}{|c|}{ Projectile: } & \multicolumn{4}{|c|}{0.50 cal FSP } & & \\
\hline \multicolumn{2}{|c|}{$\begin{array}{l}\text { Velocity } \\
\text { Measurement: }\end{array}$} & \multicolumn{4}{|c|}{ Xray } & & \\
\hline \multicolumn{2}{|r|}{$\mathrm{V}_{50}:$} & \multicolumn{2}{|c|}{$1929 \mathrm{ft} / \mathrm{s}$} & & \multicolumn{2}{|c|}{ Number of Shots: } & 4 \\
\hline & Std Dev: & \multicolumn{2}{|c|}{$19 \mathrm{ft} / \mathrm{s}$} & & \multicolumn{2}{|c|}{ Spread: } & $46 \mathrm{ft} / \mathrm{s}$ \\
\hline & ZMR: & \multicolumn{2}{|c|}{$46 \mathrm{ft} / \mathrm{s}$} & & & & \\
\hline \multicolumn{2}{|c|}{ Striking Velocity } & \multirow{2}{*}{$\begin{array}{l}\text { Pitch } \\
\text { (deg) }\end{array}$} & \multirow{2}{*}{$\begin{array}{l}\text { Yaw } \\
(\mathrm{deg})\end{array}$} & \multirow{2}{*}{$\begin{array}{l}\text { Result } \\
(\mathrm{PP} / \mathrm{CP})\end{array}$} & \multirow{2}{*}{$\begin{array}{c}\text { Used for } \\
\mathrm{V}_{50} \\
(\mathrm{Yes} / \mathrm{No})\end{array}$} & \multirow{2}{*}{$\begin{array}{c}\text { Shot } \\
\text { Number }\end{array}$} & \multirow[t]{2}{*}{ Comments } \\
\hline$(\mathrm{ft} / \mathrm{s})$ & $(\mathrm{m} / \mathrm{s})$ & & & & & & \\
\hline 1988 & 606 & 0.50 & -0.50 & $\mathrm{CP}$ & No & 14177 & \\
\hline 1903 & 580 & 0.50 & 0.25 & $\mathbf{C P}$ & Yes & 14178 & \\
\hline 1793 & 546 & 0.00 & 0.00 & $\mathrm{PP}$ & No & 14179 & \\
\hline 1818 & 554 & 0.50 & -0.25 & $\mathrm{PP}$ & No & 14180 & \\
\hline 1803 & 550 & -0.25 & 0.25 & PP & No & 14181 & \\
\hline 1933 & 589 & 0.50 & -0.50 & PP & Yes & 14182 & \\
\hline 1949 & 594 & 0.50 & 0.25 & PP & Yes & 14183 & \\
\hline 1932 & 589 & 0.00 & 0.25 & $\mathbf{C P}$ & Yes & 14184 & \\
\hline & & & & & & & \\
\hline & & & & & & & \\
\hline & & & & & & & \\
\hline & & & & & & & \\
\hline
\end{tabular}




\begin{tabular}{|c|c|c|c|c|c|c|c|}
\hline \multicolumn{2}{|l|}{ Target: } & \multicolumn{4}{|c|}{ 6055-T651 } & Date: & 1/9/2013 \\
\hline \multicolumn{2}{|c|}{ Plate Number: } & \multicolumn{4}{|c|}{$521-822$} & Location: & EF 110G \\
\hline \multicolumn{2}{|c|}{ Thickness, in: } & \multicolumn{4}{|c|}{0.991} & & \\
\hline \multicolumn{2}{|c|}{ Thickness, mm: } & \multicolumn{4}{|l|}{25.17} & & \\
\hline \multicolumn{2}{|c|}{ Hardness, BHN: } & \multicolumn{4}{|l|}{116} & & \\
\hline \multicolumn{2}{|c|}{ Obliquity: } & \multicolumn{4}{|l|}{$0^{\circ}$} & & \\
\hline \multicolumn{2}{|c|}{ Projectile: } & \multicolumn{4}{|c|}{0.50 cal FSP } & & \\
\hline \multicolumn{2}{|c|}{$\begin{array}{l}\text { Velocity } \\
\text { Measurement: }\end{array}$} & \multicolumn{4}{|l|}{ Xray } & & \\
\hline & $\mathrm{V}_{50}:$ & \multicolumn{2}{|c|}{$3037 \mathrm{ft} / \mathrm{s}$} & & \multicolumn{2}{|c|}{ Number of Shots: } & 4 \\
\hline & Std Dev: & \multicolumn{2}{|c|}{$28 \mathrm{ft} / \mathrm{s}$} & & \multicolumn{2}{|r|}{ Spread: } & $60 \mathrm{ft} / \mathrm{s}$ \\
\hline & ZMR: & \multicolumn{2}{|l|}{ N/A } & & & & \\
\hline \multicolumn{2}{|c|}{ Striking Velocity } & \multirow{2}{*}{$\begin{array}{l}\text { Pitch } \\
\text { (deg) }\end{array}$} & \multirow{2}{*}{$\begin{array}{l}\text { Yaw } \\
(\mathrm{deg})\end{array}$} & \multirow{2}{*}{$\begin{array}{l}\text { Result } \\
\text { (PP/CP) }\end{array}$} & \multirow{2}{*}{$\begin{array}{c}\text { Used for } \\
V_{50} \\
(\mathrm{Yes} / \mathrm{No})\end{array}$} & \multirow{2}{*}{$\begin{array}{c}\text { Shot } \\
\text { Number }\end{array}$} & \multirow[t]{2}{*}{ Comments } \\
\hline$(\mathrm{ft} / \mathrm{s})$ & $(\mathrm{m} / \mathrm{s})$ & & & & & & \\
\hline 3431 & 1046 & -0.50 & 1.00 & $\mathrm{CP}$ & No & 14149 & \\
\hline 3455 & 1053 & 1.50 & -1.00 & $\mathrm{CP}$ & No & 14150 & \\
\hline 3257 & 993 & -2.50 & -1.50 & $\mathrm{CP}$ & No & 14151 & \\
\hline 3324 & 1013 & -0.25 & -0.50 & $\mathrm{CP}$ & No & 14152 & \\
\hline 3001 & 915 & 0.00 & 2.00 & PP & Yes & 14153 & \\
\hline 3072 & 936 & -0.75 & -1.25 & $\mathrm{CP}$ & No & 14154 & \\
\hline 3069 & 935 & -0.75 & 1.00 & $\mathrm{CP}$ & No & 14155 & \\
\hline 2971 & 906 & -0.25 & 0.75 & PP & No & 14156 & \\
\hline 3026 & 922 & -0.50 & 0.50 & PP & Yes & 14157 & \\
\hline 3058 & 932 & -0.75 & $\begin{array}{l}-0.50 \\
\end{array}$ & $\mathbf{C P}$ & Yes & 14158 & \\
\hline 3061 & 933 & 0.00 & 0.25 & $\mathbf{C P}$ & Yes & 14159 & \\
\hline & & & & & & & \\
\hline
\end{tabular}




\begin{tabular}{|c|c|c|c|c|c|c|c|}
\hline \multicolumn{2}{|l|}{ Target: } & \multicolumn{4}{|c|}{ 6055-T651 } & Date: & $1 / 14 / 2013$ \\
\hline \multicolumn{2}{|c|}{ Plate Number: } & \multicolumn{4}{|c|}{$521-832$} & Location: & EF 110G \\
\hline \multicolumn{2}{|c|}{ Thickness, in: } & \multicolumn{4}{|l|}{1.010} & & \\
\hline \multicolumn{2}{|c|}{ Thickness, mm: } & \multicolumn{4}{|l|}{25.65} & & \\
\hline \multicolumn{2}{|c|}{ Hardness, BHN: } & \multicolumn{4}{|l|}{118} & & \\
\hline \multicolumn{2}{|c|}{ Obliquity: } & \multicolumn{4}{|l|}{$0^{\circ}$} & & \\
\hline \multicolumn{2}{|c|}{ Projectile: } & \multicolumn{4}{|c|}{0.50 cal FSP } & & \\
\hline \multicolumn{2}{|c|}{\begin{tabular}{|l|} 
Velocity \\
Measurement:
\end{tabular}} & \multicolumn{4}{|c|}{ Xray } & & \\
\hline \multicolumn{2}{|c|}{$\mathrm{V}_{50}:$} & \multicolumn{2}{|c|}{$3114 \mathrm{ft} / \mathrm{s}$} & & \multicolumn{2}{|c|}{ Number of Shots: } & 4 \\
\hline \multirow{2}{*}{\multicolumn{2}{|c|}{$\begin{array}{r}\text { Std Dev: } \\
\text { ZMR: }\end{array}$}} & \multicolumn{2}{|c|}{$27 \mathrm{ft} / \mathrm{s}$} & & \multicolumn{2}{|c|}{ Spread: } & $56 \mathrm{ft} / \mathrm{s}$ \\
\hline & & \multicolumn{2}{|c|}{$29 \mathrm{ft} / \mathrm{s}$} & & & & \\
\hline \multicolumn{2}{|c|}{ Striking Velocity } & \multirow{2}{*}{$\begin{array}{l}\text { Pitch } \\
\text { (deg) }\end{array}$} & \multirow{2}{*}{$\begin{array}{c}\text { Yaw } \\
(\mathrm{deg})\end{array}$} & \multirow{2}{*}{$\begin{array}{l}\text { Result } \\
(\mathrm{PP} / \mathrm{CP})\end{array}$} & \multirow{2}{*}{$\begin{array}{c}\text { Used for } \\
\mathrm{V}_{50} \\
(\mathrm{Yes} / \mathrm{No})\end{array}$} & \multirow{2}{*}{$\begin{array}{c}\text { Shot } \\
\text { Number }\end{array}$} & \multirow[t]{2}{*}{ Comments } \\
\hline$(\mathrm{ft} / \mathrm{s})$ & $(\mathrm{m} / \mathrm{s})$ & & & & & & \\
\hline 3032 & 924 & 0.00 & 0.00 & PP & No & 14171 & \\
\hline 3079 & 938 & 0.00 & 0.50 & PP & Yes & 14172 & \\
\hline 3106 & 947 & 0.25 & 0.25 & CP & Yes & 14173 & \\
\hline 2964 & 903 & -0.25 & -0.25 & $\mathrm{PP}$ & No & 14174 & \\
\hline 3134 & 955 & 0.00 & 0.75 & $\mathbf{C P}$ & Yes & 14175 & \\
\hline 3135 & 956 & 0.00 & 0.50 & PP & Yes & 14176 & \\
\hline & & & & & & & \\
\hline & & & & & & & \\
\hline & & & & & & & \\
\hline & & & & & & & \\
\hline & & & & & & & \\
\hline & & & & & & & \\
\hline
\end{tabular}




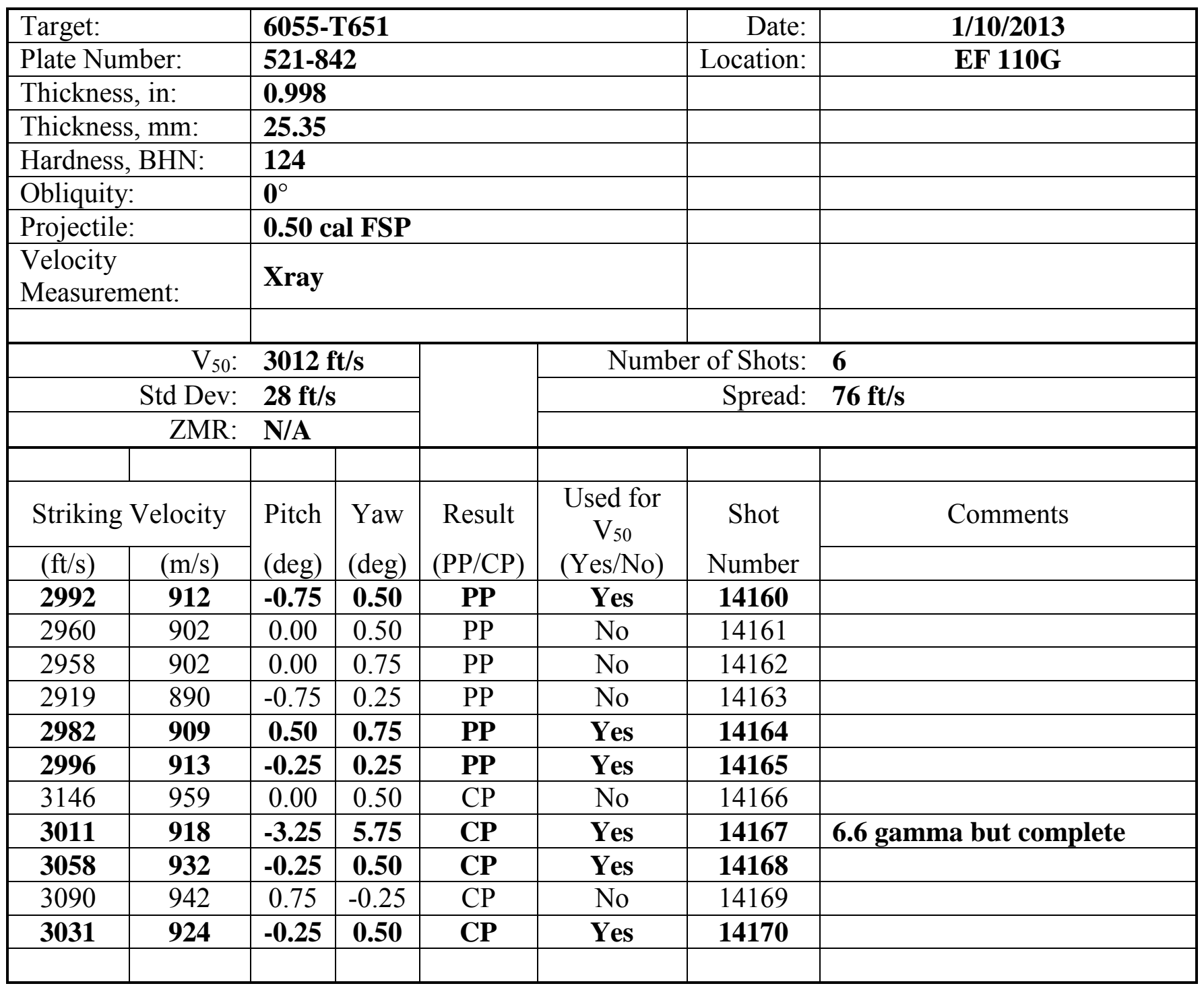




\section{0-mm FSP}

\begin{tabular}{|c|c|c|c|c|c|c|c|}
\hline \multicolumn{2}{|l|}{ Target: } & \multicolumn{4}{|c|}{ 6055-T651 } & Date: & 3/28/2013 \\
\hline \multicolumn{2}{|c|}{ Plate Number: } & \multicolumn{4}{|c|}{$521-822$} & Location: & EF 110E \\
\hline \multicolumn{2}{|c|}{ Thickness, in: } & \multicolumn{4}{|c|}{0.991} & & \\
\hline \multicolumn{2}{|c|}{ Thickness, mm: } & \multicolumn{4}{|l|}{25.17} & & \\
\hline \multicolumn{2}{|c|}{ Hardness, BHN: } & \multicolumn{4}{|l|}{116} & & \\
\hline \multicolumn{2}{|c|}{ Obliquity: } & \multicolumn{4}{|l|}{$\mathbf{0}^{\circ}$} & & \\
\hline \multicolumn{2}{|c|}{ Projectile: } & \multicolumn{4}{|c|}{ 20-mm FSP } & & \\
\hline \multicolumn{2}{|c|}{$\begin{array}{l}\text { Velocity } \\
\text { Measurement: }\end{array}$} & \multicolumn{4}{|l|}{ Xray } & & \\
\hline & $\mathrm{V}_{50}$ & \multicolumn{2}{|c|}{$1449 \mathrm{ft} / \mathrm{s}$} & & \multicolumn{2}{|c|}{ Number of Shots: } & 6 \\
\hline & Std Dev & \multicolumn{2}{|c|}{$26 \mathrm{ft} / \mathrm{s}$} & & \multicolumn{2}{|r|}{ Spread: } & $67 \mathrm{ft} / \mathrm{s}$ \\
\hline & ZMR & \multicolumn{2}{|c|}{$32 \mathrm{ft} / \mathrm{s}$} & & & & \\
\hline \multicolumn{2}{|c|}{ Striking Velocity } & \multirow{2}{*}{$\begin{array}{l}\text { Pitch } \\
(\mathrm{deg})\end{array}$} & \multirow{2}{*}{$\begin{array}{l}\text { Yaw } \\
(\mathrm{deg})\end{array}$} & \multirow{2}{*}{$\begin{array}{c}\text { Result } \\
(\mathrm{PP} / \mathrm{CP})\end{array}$} & \multirow{2}{*}{$\begin{array}{c}\text { Used for } \\
V_{50} \\
(\text { Yes/No) }\end{array}$} & \multirow{2}{*}{$\begin{array}{c}\text { Shot } \\
\text { Number }\end{array}$} & \multirow[t]{2}{*}{ Comments } \\
\hline$(\mathrm{ft} / \mathrm{s})$ & $(\mathrm{m} / \mathrm{s})$ & & & & & & \\
\hline 1640 & 500 & 0.00 & 0.25 & $\mathrm{CP}$ & No & 1962 & \\
\hline 1428 & 435 & -0.25 & -1.25 & PP & Yes & 1963 & \\
\hline 1477 & 450 & 0.00 & -2.50 & PP & Yes & 1964 & \\
\hline 1496 & 456 & -0.25 & -0.25 & $\mathrm{CP}$ & No & 1965 & \\
\hline 1495 & 456 & -1.00 & -0.75 & $\mathrm{CP}$ & No & 1966 & \\
\hline 1460 & 445 & 0.00 & -1.75 & $\mathbf{C P}$ & Yes & 1967 & \\
\hline 1473 & 449 & -0.50 & -1.25 & $\mathbf{C P}$ & Yes & 1968 & \\
\hline 1445 & 440 & 0.00 & -0.50 & CP & Yes & 1969 & \\
\hline 1410 & 430 & -0.75 & 0.25 & PP & Yes & 1970 & \\
\hline & & & & & & & \\
\hline & & & & & & & \\
\hline & & & & & & & \\
\hline
\end{tabular}




\begin{tabular}{|c|c|c|c|c|c|c|c|}
\hline \multicolumn{2}{|c|}{ Target: } & \multicolumn{4}{|c|}{ 6055-T651 } & Date: & 4/1/2013 \\
\hline \multicolumn{2}{|c|}{ Plate Number: } & \multicolumn{4}{|c|}{$521-832$} & Location: & EF 110E \\
\hline \multicolumn{2}{|c|}{ Thickness, in: } & \multicolumn{4}{|c|}{1.002} & & \\
\hline \multicolumn{2}{|c|}{ Thickness, mm: } & \multicolumn{4}{|l|}{25.45} & & \\
\hline \multicolumn{2}{|c|}{ Hardness, BHN: } & \multicolumn{4}{|l|}{114} & & \\
\hline \multicolumn{2}{|c|}{ Obliquity: } & \multicolumn{4}{|l|}{$0^{\circ}$} & & \\
\hline \multicolumn{2}{|c|}{ Projectile: } & \multicolumn{4}{|c|}{ 20-mm FSP } & & \\
\hline \multicolumn{2}{|c|}{$\begin{array}{l}\text { Velocity } \\
\text { Measurement: }\end{array}$} & \multicolumn{4}{|l|}{ Xray } & & \\
\hline \multicolumn{2}{|c|}{$\mathrm{V}_{50}:$} & \multicolumn{2}{|c|}{$1372 \mathrm{ft} / \mathrm{s}$} & & \multicolumn{2}{|c|}{ Number of Shots: } & 4 \\
\hline & Std Dev & \multicolumn{2}{|c|}{$16 \mathrm{ft} / \mathrm{s}$} & & \multicolumn{2}{|r|}{ Spread: } & $37 \mathrm{ft} / \mathrm{s}$ \\
\hline & ZMR & \multicolumn{2}{|l|}{ N/A } & & & & \\
\hline \multicolumn{2}{|c|}{ Striking Velocity } & \multirow{2}{*}{$\begin{array}{l}\text { Pitch } \\
\text { (deg) }\end{array}$} & \multirow{2}{*}{$\begin{array}{l}\text { Yaw } \\
(\mathrm{deg})\end{array}$} & \multirow{2}{*}{$\begin{array}{l}\text { Result } \\
\text { (PP/CP) }\end{array}$} & \multirow{2}{*}{$\begin{array}{c}\text { Used for } \\
V_{50} \\
(\mathrm{Yes} / \mathrm{No})\end{array}$} & \multirow{2}{*}{$\begin{array}{c}\text { Shot } \\
\text { Number }\end{array}$} & \multirow[t]{2}{*}{ Comments } \\
\hline$(\mathrm{ft} / \mathrm{s})$ & $(\mathrm{m} / \mathrm{s})$ & & & & & & \\
\hline 1458 & 444 & 0.00 & -0.50 & $\mathrm{CP}$ & No & 1971 & \\
\hline 1388 & 423 & -0.50 & 0.25 & $\mathbf{C P}$ & Yes & 1972 & \\
\hline 1366 & 416 & -0.50 & -0.75 & PP & Yes & 1973 & \\
\hline 1381 & 421 & 0.25 & -0.50 & $\mathbf{C P}$ & Yes & 1974 & \\
\hline 1351 & 412 & 0.25 & 1.00 & $\mathbf{P P}$ & Yes & 1975 & \\
\hline & & & & & & & \\
\hline & & & & & & & \\
\hline & & & & & & & \\
\hline & & & & & & & \\
\hline & & & & & & & \\
\hline & & & & & & & \\
\hline
\end{tabular}




\begin{tabular}{|c|c|c|c|c|c|c|c|}
\hline \multicolumn{2}{|c|}{ Target: } & \multicolumn{4}{|c|}{ 6055-T651 } & Date: & 4/1/2013 \\
\hline \multicolumn{2}{|c|}{ Plate Number: } & \multicolumn{4}{|c|}{$521-842$} & Location: & EF 110E \\
\hline \multicolumn{2}{|c|}{ Thickness, in: } & \multicolumn{4}{|c|}{1.000} & & \\
\hline \multicolumn{2}{|c|}{ Thickness, mm: } & \multicolumn{4}{|l|}{25.40} & & \\
\hline \multicolumn{2}{|c|}{ Hardness, BHN: } & \multicolumn{4}{|l|}{121} & & \\
\hline \multicolumn{2}{|c|}{ Obliquity: } & \multicolumn{4}{|l|}{$0^{\circ}$} & & \\
\hline \multicolumn{2}{|c|}{ Projectile: } & \multicolumn{4}{|c|}{ 20-mm FSP } & & \\
\hline \multicolumn{2}{|c|}{$\begin{array}{l}\text { Velocity } \\
\text { Measurement: }\end{array}$} & \multicolumn{4}{|l|}{ Xray } & & \\
\hline \multicolumn{2}{|c|}{$\mathrm{V}_{50}:$} & \multicolumn{2}{|c|}{$1384 \mathrm{ft} / \mathrm{s}$} & & \multicolumn{2}{|c|}{ Number of Shots: } & 4 \\
\hline & Std Dev & \multicolumn{2}{|c|}{$17 \mathrm{ft} / \mathrm{s}$} & & \multicolumn{2}{|r|}{ Spread: } & $39 \mathrm{ft} / \mathrm{s}$ \\
\hline & ZMR & \multicolumn{2}{|l|}{ N/A } & & & & \\
\hline \multicolumn{2}{|c|}{ Striking Velocity } & \multirow{2}{*}{$\begin{array}{l}\text { Pitch } \\
\text { (deg) }\end{array}$} & \multirow{2}{*}{$\begin{array}{l}\text { Yaw } \\
(\mathrm{deg})\end{array}$} & \multirow{2}{*}{$\begin{array}{l}\text { Result } \\
\text { (PP/CP) }\end{array}$} & \multirow{2}{*}{$\begin{array}{c}\text { Used for } \\
\mathrm{V}_{50} \\
\text { (Yes/No) }\end{array}$} & \multirow{2}{*}{$\begin{array}{c}\text { Shot } \\
\text { Number }\end{array}$} & \multirow[t]{2}{*}{ Comments } \\
\hline$(\mathrm{ft} / \mathrm{s})$ & $(\mathrm{m} / \mathrm{s})$ & & & & & & \\
\hline 1363 & 415 & 0.25 & 0.50 & $\mathbf{P P}$ & Yes & 1976 & \\
\hline 1391 & 424 & 0.00 & 0.00 & PP & Yes & 1977 & \\
\hline 1379 & 420 & -0.50 & -0.50 & $\mathbf{C P}$ & Yes & 1978 & \\
\hline 1402 & 427 & -0.50 & -2.00 & $\mathbf{C P}$ & Yes & 1979 & \\
\hline & & & & & & & \\
\hline & & & & & & & \\
\hline & & & & & & & \\
\hline & & & & & & & \\
\hline & & & & & & & \\
\hline & & & & & & & \\
\hline & & & & & & & \\
\hline
\end{tabular}




\begin{tabular}{|c|c|c|c|c|c|c|c|}
\hline \multicolumn{2}{|c|}{ Target: } & \multicolumn{4}{|c|}{ 6055-T651 } & Date: & $4 / 4 / 2013$ \\
\hline \multicolumn{2}{|c|}{ Plate Number: } & \multicolumn{4}{|c|}{ 521-871 } & Location: & EF 110E \\
\hline \multicolumn{2}{|c|}{ Thickness, in: } & \multicolumn{4}{|c|}{1.493} & & \\
\hline \multicolumn{2}{|c|}{ Thickness, mm: } & \multicolumn{4}{|l|}{37.92} & & \\
\hline \multicolumn{2}{|c|}{ Hardness, BHN: } & \multicolumn{4}{|l|}{112} & & \\
\hline \multicolumn{2}{|c|}{ Obliquity: } & \multicolumn{4}{|l|}{$0^{\circ}$} & & \\
\hline \multicolumn{2}{|c|}{ Projectile: } & \multicolumn{4}{|c|}{ 20-mm FSP } & & \\
\hline \multicolumn{2}{|c|}{$\begin{array}{l}\text { Velocity } \\
\text { Measurement: }\end{array}$} & \multicolumn{4}{|l|}{ Xray } & & \\
\hline \multicolumn{2}{|r|}{$\mathrm{V}_{50}:$} & \multicolumn{2}{|c|}{$2661 \mathrm{ft} / \mathrm{s}$} & & \multicolumn{2}{|c|}{ Number of Shots: } & 4 \\
\hline & Std Dev: & \multicolumn{2}{|c|}{$26 \mathrm{ft} / \mathrm{s}$} & & \multicolumn{2}{|r|}{ Spread: } & $60 \mathrm{ft} / \mathrm{s}$ \\
\hline & ZMR: & \multicolumn{2}{|l|}{ N/A } & & & & \\
\hline \multicolumn{2}{|c|}{ Striking Velocity } & \multirow{2}{*}{$\begin{array}{l}\text { Pitch } \\
\text { (deg) }\end{array}$} & \multirow{2}{*}{$\begin{array}{l}\text { Yaw } \\
(\mathrm{deg})\end{array}$} & \multirow{2}{*}{$\begin{array}{l}\text { Result } \\
\text { (PP/CP) }\end{array}$} & \multirow{2}{*}{$\begin{array}{c}\text { Used for } \\
V_{50} \\
(\mathrm{Yes} / \mathrm{No})\end{array}$} & \multirow{2}{*}{$\begin{array}{c}\text { Shot } \\
\text { Number }\end{array}$} & \multirow[t]{2}{*}{ Comments } \\
\hline$(\mathrm{ft} / \mathrm{s})$ & $(\mathrm{m} / \mathrm{s})$ & & & & & & \\
\hline 2698 & 822 & -0.50 & 1.25 & $\mathbf{C P}$ & Yes & 1990 & \\
\hline 2630 & 802 & 0.00 & 0.25 & PP & No & 1991 & \\
\hline 2638 & 804 & -0.50 & 0.75 & PP & Yes & 1992 & \\
\hline 2657 & 810 & 0.00 & 0.00 & $\mathbf{C P}$ & Yes & 1993 & \\
\hline 2649 & 807 & 0.25 & 0.25 & $\mathbf{P P}$ & Yes & 1994 & \\
\hline & & & & & & & \\
\hline & & & & & & & \\
\hline & & & & & & & \\
\hline & & & & & & & \\
\hline & & & & & & & \\
\hline & & & & & & & \\
\hline
\end{tabular}




\begin{tabular}{|c|c|c|c|c|c|c|c|}
\hline \multicolumn{2}{|l|}{ Target: } & \multicolumn{4}{|c|}{ 6055-T651 } & Date: & $4 / 3 / 2013$ \\
\hline \multicolumn{2}{|c|}{ Plate Number: } & \multicolumn{4}{|c|}{$521-881$} & Location: & EF 110E \\
\hline \multicolumn{2}{|c|}{ Thickness, in: } & \multicolumn{4}{|c|}{1.497} & & \\
\hline \multicolumn{2}{|c|}{ Thickness, mm: } & \multicolumn{4}{|l|}{38.02} & & \\
\hline \multicolumn{2}{|c|}{ Hardness, BHN: } & \multicolumn{4}{|l|}{121} & & \\
\hline \multicolumn{2}{|c|}{ Obliquity: } & \multicolumn{4}{|l|}{$0^{\circ}$} & & \\
\hline \multicolumn{2}{|c|}{ Projectile: } & \multicolumn{4}{|c|}{ 20-mm FSP } & & \\
\hline \multicolumn{2}{|c|}{$\begin{array}{l}\text { Velocity } \\
\text { Measurement: }\end{array}$} & \multicolumn{4}{|l|}{ Xray } & & \\
\hline \multicolumn{2}{|r|}{$\mathrm{V}_{50}$ : } & \multicolumn{2}{|c|}{$2681 \mathrm{ft} / \mathrm{s}$} & & \multicolumn{2}{|c|}{ Number of Shots: } & 4 \\
\hline & Std Dev: & \multicolumn{2}{|c|}{$12 \mathrm{ft} / \mathrm{s}$} & & \multicolumn{2}{|r|}{ Spread: } & $29 \mathrm{ft} / \mathrm{s}$ \\
\hline & ZMR: & \multicolumn{2}{|l|}{ N/A } & & & & \\
\hline \multicolumn{2}{|c|}{ Striking Velocity } & \multirow{2}{*}{$\begin{array}{l}\text { Pitch } \\
\text { (deg) }\end{array}$} & \multirow{2}{*}{$\begin{array}{l}\text { Yaw } \\
(\mathrm{deg})\end{array}$} & \multirow{2}{*}{$\begin{array}{l}\text { Result } \\
\text { (PP/CP) }\end{array}$} & \multirow{2}{*}{$\begin{array}{c}\text { Used for } \\
V_{50} \\
(\mathrm{Yes} / \mathrm{No})\end{array}$} & \multirow{2}{*}{$\begin{array}{c}\text { Shot } \\
\text { Number }\end{array}$} & \multirow[t]{2}{*}{ Comments } \\
\hline$(\mathrm{ft} / \mathrm{s})$ & $(\mathrm{m} / \mathrm{s})$ & & & & & & \\
\hline 3029 & 923 & 0.25 & 0.25 & $\mathrm{CP}$ & No & 1980 & \\
\hline 2981 & 909 & -0.25 & 0.00 & $\mathrm{CP}$ & No & 1981 & \\
\hline 2907 & 886 & 0.00 & 0.25 & $\mathrm{CP}$ & No & 1982 & \\
\hline 2734 & 833 & -0.50 & 1.25 & $\mathrm{CP}$ & No & 1983 & \\
\hline 2611 & 796 & -0.25 & 0.50 & PP & No & 1984 & \\
\hline 2668 & 813 & 0.00 & 0.00 & PP & Yes & 1985 & \\
\hline 2680 & 817 & -0.75 & 1.00 & $\mathbf{C P}$ & Yes & 1986 & \\
\hline 2679 & 817 & -0.25 & 1.75 & $\mathbf{P P}$ & Yes & 1987 & \\
\hline 2663 & 812 & -0.25 & -1.50 & PP & No & 1988 & \\
\hline 2697 & 822 & 0.00 & 0.25 & $\mathbf{C P}$ & Yes & 1989 & \\
\hline & & & & & & & \\
\hline & & & & & & & \\
\hline
\end{tabular}




\begin{tabular}{|c|c|c|c|c|c|c|c|}
\hline \multicolumn{2}{|l|}{ Target: } & \multicolumn{4}{|c|}{ 6055-T651 } & Date: & 4/10/2013 \\
\hline \multicolumn{2}{|c|}{ Plate Number: } & \multicolumn{4}{|c|}{$521-882$} & Location: & EF 110E \\
\hline \multicolumn{2}{|c|}{ Thickness, in: } & \multicolumn{4}{|c|}{1.493} & & \\
\hline \multicolumn{2}{|c|}{ Thickness, mm: } & \multicolumn{4}{|l|}{37.92} & & \\
\hline \multicolumn{2}{|c|}{ Hardness, BHN: } & \multicolumn{4}{|l|}{112} & & \\
\hline \multicolumn{2}{|c|}{ Obliquity: } & \multicolumn{4}{|l|}{$0^{\circ}$} & & \\
\hline \multicolumn{2}{|c|}{ Projectile: } & \multicolumn{4}{|c|}{ 20-mm FSP } & & \\
\hline \multicolumn{2}{|c|}{$\begin{array}{l}\text { Velocity } \\
\text { Measurement: }\end{array}$} & \multicolumn{4}{|l|}{ Xray } & & \\
\hline & $\mathrm{V}_{50}:$ & \multicolumn{2}{|c|}{$2565 \mathrm{ft} / \mathrm{s}$} & & \multicolumn{2}{|c|}{ Number of Shots: } & 6 \\
\hline & Std Dev: & \multicolumn{2}{|c|}{$24 \mathrm{ft} / \mathrm{s}$} & & \multicolumn{2}{|r|}{ Spread: } & $65 \mathrm{ft} / \mathrm{s}$ \\
\hline & ZMR: & \multicolumn{2}{|c|}{$31 \mathrm{ft} / \mathrm{s}$} & & & & \\
\hline \multicolumn{2}{|c|}{ Striking Velocity } & \multirow{2}{*}{$\begin{array}{l}\text { Pitch } \\
\text { (deg) }\end{array}$} & \multirow{2}{*}{$\begin{array}{l}\text { Yaw } \\
\text { (deg) }\end{array}$} & \multirow{2}{*}{$\begin{array}{l}\text { Result } \\
\text { (PP/CP) }\end{array}$} & \multirow{2}{*}{$\begin{array}{c}\text { Used for } \\
V_{50} \\
(\text { Yes/No) }\end{array}$} & \multirow{2}{*}{$\begin{array}{c}\text { Shot } \\
\text { Number }\end{array}$} & \multirow[t]{2}{*}{ Comments } \\
\hline$(\mathrm{ft} / \mathrm{s})$ & $(\mathrm{m} / \mathrm{s})$ & & & & & & \\
\hline 2637 & 804 & -0.75 & 0.75 & $\mathrm{CP}$ & No & 1995 & \\
\hline 2585 & 788 & 0.00 & -0.25 & PP & Yes & 1996 & \\
\hline 2599 & 792 & -0.50 & 0.00 & $\mathrm{CP}$ & No & 1997 & \\
\hline 2610 & 795 & -0.25 & 1.00 & $\mathrm{CP}$ & No & 1998 & \\
\hline 2588 & 789 & 0.00 & -0.50 & $\mathbf{C P}$ & Yes & 1999 & \\
\hline 2593 & 790 & -0.75 & 1.00 & $\mathrm{CP}$ & No & 2000 & \\
\hline 2602 & 793 & -1.25 & 0.75 & $\mathrm{CP}$ & No & 2001 & \\
\hline 2568 & 783 & -0.50 & -0.25 & $\mathbf{C P}$ & Yes & 2002 & \\
\hline 2554 & 778 & 0.00 & 0.00 & $\mathbf{C P}$ & Yes & 2003 & \\
\hline 2523 & 769 & 0.00 & 0.00 & PP & Yes & 2004 & \\
\hline 2571 & 784 & -0.50 & 0.00 & PP & Yes & 14430 & \\
\hline & & & & & & & \\
\hline
\end{tabular}




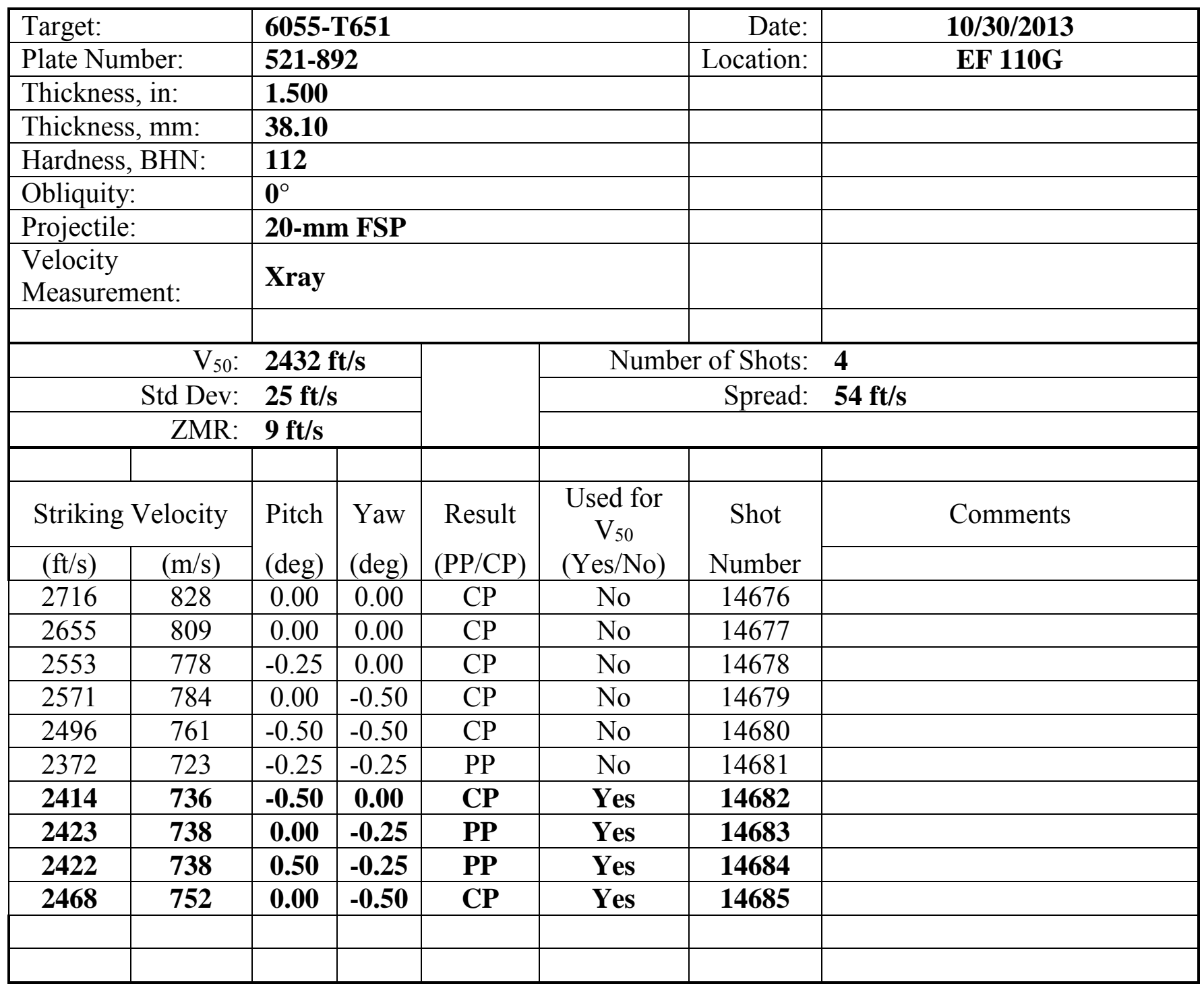




\begin{tabular}{|c|c|c|c|c|c|c|c|}
\hline \multirow{2}{*}{\multicolumn{2}{|c|}{$\begin{array}{l}\text { Target: } \\
\text { Plate Number: }\end{array}$}} & \multicolumn{4}{|c|}{ 6055-T651 } & Date: & $4 / 25 / 2013$ \\
\hline & & 521-9 & & & & Location: & EF 110G \\
\hline \multicolumn{2}{|c|}{ Thickness, in: } & \multicolumn{4}{|c|}{1.997} & & \\
\hline \multicolumn{2}{|c|}{ Thickness, mm: } & \multicolumn{4}{|l|}{50.72} & & \\
\hline \multicolumn{2}{|c|}{ Hardness, BHN: } & \multicolumn{4}{|l|}{101} & & \\
\hline \multicolumn{2}{|c|}{ Obliquity: } & \multicolumn{4}{|l|}{$0^{\circ}$} & & \\
\hline \multicolumn{2}{|c|}{ Projectile: } & \multicolumn{4}{|c|}{ 20-mm FSP } & & \\
\hline \multicolumn{2}{|c|}{$\begin{array}{l}\text { Velocity } \\
\text { Measurement: }\end{array}$} & \multicolumn{4}{|c|}{ Xray } & & \\
\hline \multicolumn{2}{|c|}{$\mathrm{V}_{50}:$} & \multicolumn{2}{|c|}{$3985 \mathrm{ft} / \mathrm{s}$} & & \multicolumn{2}{|c|}{ Number of Shots: } & 6 \\
\hline & Std Dev: & \multicolumn{2}{|c|}{$30 \mathrm{ft} / \mathrm{s}$} & & \multicolumn{2}{|c|}{ Spread: } & $78 \mathrm{ft} / \mathrm{s}$ \\
\hline & ZMR: & \multicolumn{2}{|c|}{ N/A } & & & & \\
\hline \multicolumn{2}{|c|}{ Striking Velocity } & \multirow{2}{*}{$\begin{array}{l}\text { Pitch } \\
(\mathrm{deg})\end{array}$} & \multirow{2}{*}{$\begin{array}{l}\text { Yaw } \\
(\mathrm{deg})\end{array}$} & \multirow{2}{*}{$\begin{array}{l}\text { Result } \\
(\mathrm{PP} / \mathrm{CP})\end{array}$} & \multirow{2}{*}{$\begin{array}{c}\text { Used for } \\
\mathrm{V}_{50} \\
(\mathrm{Yes} / \mathrm{No})\end{array}$} & \multirow{2}{*}{$\begin{array}{c}\text { Shot } \\
\text { Number }\end{array}$} & \multirow[t]{2}{*}{ Comments } \\
\hline$(\mathrm{ft} / \mathrm{s})$ & $(\mathrm{m} / \mathrm{s})$ & & & & & & \\
\hline 3935 & 1199 & 0.00 & -0.25 & $\mathbf{P P}$ & Yes & 14417 & \\
\hline 4006 & 1221 & 0.25 & 0.25 & $\mathbf{C P}$ & Yes & 14418 & \\
\hline 3979 & 1213 & 0.25 & -0.50 & $\mathbf{P P}$ & Yes & 14419 & \\
\hline 3969 & 1210 & -0.25 & 0.00 & $\mathbf{P P}$ & Yes & 14420 & \\
\hline 4010 & 1222 & 0.25 & -0.50 & $\mathbf{C P}$ & Yes & 14421 & \\
\hline 4013 & 1223 & -0.25 & 0.25 & CP & Yes & 14422 & \\
\hline & & & & & & & \\
\hline & & & & & & & \\
\hline & & & & & & & \\
\hline & & & & & & & \\
\hline & & & & & & & \\
\hline
\end{tabular}




\begin{tabular}{|c|c|c|c|c|c|c|c|}
\hline \multicolumn{2}{|c|}{ Target: } & \multicolumn{4}{|c|}{ 6055-T651 } & Date: & $10 / 24 / 2013$ \\
\hline \multicolumn{2}{|c|}{ Plate Number: } & \multicolumn{4}{|c|}{ 521-902 } & Location: & EF 110G \\
\hline \multicolumn{2}{|c|}{ Thickness, in: } & \multicolumn{4}{|c|}{1.984} & & \\
\hline \multicolumn{2}{|c|}{ Thickness, mm: } & \multicolumn{4}{|l|}{50.39} & & \\
\hline \multicolumn{2}{|c|}{ Hardness, BHN: } & \multicolumn{4}{|l|}{107} & & \\
\hline \multicolumn{2}{|c|}{ Obliquity: } & \multicolumn{4}{|l|}{$0^{\circ}$} & & \\
\hline \multicolumn{2}{|c|}{ Projectile: } & \multicolumn{4}{|c|}{ 20-mm FSP } & & \\
\hline \multicolumn{2}{|c|}{$\begin{array}{l}\text { Velocity } \\
\text { Measurement: }\end{array}$} & \multicolumn{4}{|l|}{ Xray } & & \\
\hline & $\mathrm{V}_{50}$ & \multicolumn{2}{|c|}{$3935 \mathrm{ft} / \mathrm{s}$} & & \multicolumn{2}{|c|}{ Number of Shots: } & 4 \\
\hline & Std Dev & \multicolumn{2}{|c|}{$31 \mathrm{ft} / \mathrm{s}$} & & \multicolumn{2}{|r|}{ Spread: } & $60 \mathrm{ft} / \mathrm{s}$ \\
\hline & ZMR & \multicolumn{2}{|c|}{$48 \mathrm{ft} / \mathrm{s}$} & & & & \\
\hline \multicolumn{2}{|c|}{ Striking Velocity } & \multirow{2}{*}{$\begin{array}{l}\text { Pitch } \\
\text { (deg) }\end{array}$} & \multirow{2}{*}{$\begin{array}{l}\text { Yaw } \\
(\mathrm{deg})\end{array}$} & \multirow{2}{*}{$\begin{array}{l}\text { Result } \\
(\mathrm{PP} / \mathrm{CP})\end{array}$} & \multirow{2}{*}{$\begin{array}{c}\text { Used for } \\
V_{50} \\
(\mathrm{Yes} / \mathrm{No})\end{array}$} & \multirow{2}{*}{$\begin{array}{c}\text { Shot } \\
\text { Number }\end{array}$} & \multirow[t]{2}{*}{ Comments } \\
\hline$(\mathrm{ft} / \mathrm{s})$ & $(\mathrm{m} / \mathrm{s})$ & & & & & & \\
\hline 3962 & 1208 & 0.00 & -0.25 & $\mathbf{P P}$ & Yes & 14653 & \\
\hline 4001 & 1219 & -0.25 & 0.25 & $\mathrm{CP}$ & No & 14654 & \\
\hline 3960 & 1207 & 0.00 & 0.50 & CP & Yes & 14655 & \\
\hline 3902 & 1189 & 0.00 & -0.50 & $\mathbf{P P}$ & Yes & 14656 & \\
\hline 3914 & 1193 & -0.75 & 0.75 & $\mathbf{C P}$ & Yes & 14657 & \\
\hline & & & & & & & \\
\hline & & & & & & & \\
\hline & & & & & & & \\
\hline & & & & & & & \\
\hline & & & & & & & \\
\hline & & & & & & & \\
\hline
\end{tabular}




\begin{tabular}{|c|c|c|c|c|c|c|c|}
\hline \multirow{2}{*}{\multicolumn{2}{|c|}{$\begin{array}{l}\text { Target: } \\
\text { Plate Number: }\end{array}$}} & \multicolumn{4}{|c|}{ 6055-T651 } & Date: & $4 / 22 / 2013$ \\
\hline & & $521-9$ & & & & Location: & EF 110G \\
\hline \multicolumn{2}{|c|}{ Thickness, in: } & \multicolumn{4}{|c|}{1.999} & & \\
\hline \multicolumn{2}{|c|}{ Thickness, mm: } & \multicolumn{4}{|l|}{50.77} & & \\
\hline \multicolumn{2}{|c|}{ Hardness, BHN: } & \multicolumn{4}{|l|}{107} & & \\
\hline \multicolumn{2}{|c|}{ Obliquity: } & \multicolumn{4}{|l|}{$0^{\circ}$} & & \\
\hline \multicolumn{2}{|c|}{ Projectile: } & \multicolumn{4}{|c|}{ 20-mm FSP } & & \\
\hline \multicolumn{2}{|c|}{\begin{tabular}{|l|} 
Velocity \\
Measurement:
\end{tabular}} & \multicolumn{4}{|c|}{ Xray } & & \\
\hline \multicolumn{2}{|r|}{$\mathrm{V}_{50}$ : } & \multicolumn{2}{|c|}{$3943 \mathrm{ft} / \mathrm{s}$} & & \multicolumn{2}{|c|}{ Number of Shots: } & 4 \\
\hline \multirow{2}{*}{\multicolumn{2}{|c|}{$\begin{array}{l}\text { Std Dev: } \\
\text { ZMR: }\end{array}$}} & \multicolumn{2}{|c|}{$24 \mathrm{ft} / \mathrm{s}$} & & \multicolumn{2}{|c|}{ Spread: } & $57 \mathrm{ft} / \mathrm{s}$ \\
\hline & & \multicolumn{2}{|l|}{ N/A } & & & & \\
\hline \multicolumn{2}{|c|}{ Striking Velocity } & \multirow{2}{*}{$\begin{array}{l}\text { Pitch } \\
\text { (deg) }\end{array}$} & \multirow{2}{*}{$\begin{array}{l}\text { Yaw } \\
(\mathrm{deg})\end{array}$} & \multirow{2}{*}{$\begin{array}{l}\text { Result } \\
\text { (PP/CP) }\end{array}$} & \multirow{2}{*}{$\begin{array}{c}\text { Used for } \\
\mathrm{V}_{50} \\
(\mathrm{Yes} / \mathrm{No})\end{array}$} & \multirow{2}{*}{$\begin{array}{c}\text { Shot } \\
\text { Number }\end{array}$} & \multirow[t]{2}{*}{ Comments } \\
\hline$(\mathrm{ft} / \mathrm{s})$ & $(\mathrm{m} / \mathrm{s})$ & & & & & & \\
\hline 3844 & 1172 & 0.25 & 0.50 & PP & No & 14402 & \\
\hline 3883 & 1183 & 0.25 & 0.00 & PP & No & 14403 & \\
\hline 3914 & 1193 & -0.50 & -0.25 & PP & Yes & 14404 & \\
\hline 3953 & 1205 & -0.25 & -1.00 & $\mathbf{C P}$ & Yes & 14405 & \\
\hline 3935 & 1199 & 0.00 & -0.25 & PP & Yes & 14406 & \\
\hline 3971 & 1210 & -0.25 & 0.75 & CP & Yes & 14407 & \\
\hline & & & & & & & \\
\hline & & & & & & & \\
\hline & & & & & & & \\
\hline & & & & & & & \\
\hline & & & & & & & \\
\hline & & & & & & & \\
\hline
\end{tabular}




\begin{tabular}{|c|c|c|c|c|c|c|c|}
\hline \multicolumn{2}{|c|}{ Target: } & \multicolumn{4}{|c|}{ 6055-T651 } & Date: & 4/18/2013 \\
\hline \multicolumn{2}{|c|}{ Plate Number: } & \multicolumn{4}{|c|}{$521-921$} & Location: & EF 110G \\
\hline \multicolumn{2}{|c|}{ Thickness, in: } & \multicolumn{4}{|c|}{2.004} & & \\
\hline \multicolumn{2}{|c|}{ Thickness, mm: } & \multicolumn{4}{|l|}{50.90} & & \\
\hline \multicolumn{2}{|c|}{ Hardness, BHN: } & \multicolumn{4}{|l|}{107} & & \\
\hline \multicolumn{2}{|c|}{ Obliquity: } & \multicolumn{4}{|l|}{$0^{\circ}$} & & \\
\hline \multicolumn{2}{|c|}{ Projectile: } & \multicolumn{4}{|c|}{ 20-mm FSP } & & \\
\hline \multicolumn{2}{|c|}{$\begin{array}{l}\text { Velocity } \\
\text { Measurement: }\end{array}$} & \multicolumn{4}{|l|}{ Xray } & & \\
\hline & $\mathrm{V}_{50}:$ & \multicolumn{2}{|c|}{$3836 \mathrm{ft} / \mathrm{s}$} & & \multicolumn{2}{|c|}{ Number of Shots: } & 4 \\
\hline & Std Dev: & \multicolumn{2}{|c|}{$16 \mathrm{ft} / \mathrm{s}$} & & \multicolumn{2}{|r|}{ Spread: } & $39 \mathrm{ft} / \mathrm{s}$ \\
\hline & ZMR: & \multicolumn{2}{|l|}{$2 \mathrm{ft} / \mathrm{s}$} & & & & \\
\hline \multicolumn{2}{|c|}{ Striking Velocity } & \multirow{2}{*}{$\begin{array}{l}\text { Pitch } \\
\text { (deg) }\end{array}$} & \multirow{2}{*}{$\begin{array}{l}\text { Yaw } \\
(\mathrm{deg})\end{array}$} & \multirow{2}{*}{$\begin{array}{l}\text { Result } \\
\text { (PP/CP) }\end{array}$} & \multirow{2}{*}{$\begin{array}{c}\text { Used for } \\
V_{50} \\
(\mathrm{Yes} / \mathrm{No})\end{array}$} & \multirow{2}{*}{$\begin{array}{c}\text { Shot } \\
\text { Number }\end{array}$} & \multirow[t]{2}{*}{ Comments } \\
\hline$(\mathrm{ft} / \mathrm{s})$ & $(\mathrm{m} / \mathrm{s})$ & & & & & & \\
\hline 3814 & 1162 & -0.25 & -0.25 & $\mathbf{P P}$ & Yes & 14398 & \\
\hline 3840 & 1170 & 0.00 & 0.00 & PP & Yes & 14399 & \\
\hline 3853 & 1174 & 0.00 & 0.25 & $\mathbf{C P}$ & Yes & 14400 & \\
\hline 3838 & 1170 & 0.00 & 0.00 & $\mathbf{C P}$ & Yes & 14401 & \\
\hline & & & & & & & \\
\hline & & & & & & & \\
\hline & & & & & & & \\
\hline & & & & & & & \\
\hline & & & & & & & \\
\hline & & & & & & & \\
\hline & & & & & & & \\
\hline
\end{tabular}


INTENTIONALLY LEFT BLANK. 
Appendix B. Additional Ballistic Test Data

This appendix appears in its original form, without editorial change. 
The following tables list data collected by ATC. ${ }^{7}$

0.30 cal. APM2, $30^{\circ}$ Obliquity Ballistic Performance

\begin{tabular}{|c|c|c|c|c|c|c|c|c|c|c|}
\hline \multirow[t]{2}{*}{ Plate ID } & \multicolumn{2}{|c|}{$\begin{array}{l}\text { Nominal } \\
\text { Thickness }\end{array}$} & \multicolumn{2}{|c|}{$\begin{array}{c}\text { Actual } \\
\text { Thickness }\end{array}$} & \multicolumn{2}{|c|}{$\begin{array}{c}\text { Areal } \\
\text { Density }\end{array}$} & \multicolumn{2}{|c|}{$\begin{array}{l}\text { Ballistic } \\
\text { Limit }\end{array}$} & \multicolumn{2}{|c|}{$\begin{array}{l}\text { Standard } \\
\text { Deviation }\end{array}$} \\
\hline & $\mathbf{m m}$ & in & $\mathbf{m m}$ & in & $\mathrm{kg} / \mathrm{m}^{2}$ & psf & $\mathrm{m} / \mathrm{s}$ & fps & $\mathrm{m} / \mathrm{s}$ & fps \\
\hline $521-802$ & 19.05 & 0.75 & 19.05 & 0.750 & 51.67 & 10.58 & 592 & 1942 & 4 & 13 \\
\hline $521-812$ & 19.05 & 0.75 & 19.02 & 0.749 & 51.60 & 10.57 & 576 & 1889 & 7 & 24 \\
\hline
\end{tabular}

0.30 cal. APM2, $0^{\circ}$ Obliquity Ballistic Performance

\begin{tabular}{|c|c|c|c|c|c|c|c|c|c|}
\hline \multirow[t]{2}{*}{ Plate ID } & \multicolumn{2}{|c|}{$\begin{array}{l}\text { Nominal } \\
\text { Thickness }\end{array}$} & \multicolumn{2}{|c|}{$\begin{array}{c}\text { Actual } \\
\text { Thickness }\end{array}$} & $\begin{array}{c}\text { Areal } \\
\text { Density }\end{array}$ & \multicolumn{2}{|c|}{$\begin{array}{c}\text { Ballistic } \\
\text { Limit }\end{array}$} & \multicolumn{2}{|c|}{$\begin{array}{l}\text { Standard } \\
\text { Deviation }\end{array}$} \\
\hline & mm & in & mm & in & $\mathrm{kg} / \mathrm{m}^{2}$ psf & $\mathbf{m} / \mathbf{s}$ & fps & $\mathrm{m} / \mathrm{s}$ & fps \\
\hline $521-801$ & 19.05 & 0.75 & 19.02 & 0.749 & 51.6010 .57 & 543 & 1780 & 9 & 29 \\
\hline $521-811$ & 19.05 & 0.75 & 19.05 & 0.750 & 51.6710 .58 & 532 & 1744 & 12 & 41 \\
\hline $521-821$ & 25.40 & 1.00 & 25.60 & 1.008 & 69.4414 .22 & 616 & 2022 & 8 & 26 \\
\hline $521-822$ & 25.40 & 1.00 & 25.60 & 1.008 & 69.4414 .22 & 603 & 1977 & 10 & 34 \\
\hline $521-831$ & 25.40 & 1.00 & 25.40 & 1.000 & 68.8914 .11 & 637 & 2089 & 3 & 11 \\
\hline $521-841$ & 25.40 & 1.00 & 25.53 & 1.005 & 69.2414 .18 & 628 & 2062 & 5 & 18 \\
\hline $521-842$ & 25.40 & 1.00 & 25.65 & 1.010 & 69.5814 .25 & 622 & 2042 & 10 & 32 \\
\hline $521-852$ & 31.75 & 1.25 & 31.50 & 1.240 & 85.4217 .50 & 694 & 2278 & 8 & 26 \\
\hline $521-861$ & 31.75 & 1.25 & 31.75 & 1.250 & 86.1117 .64 & 717 & 2354 & 7 & 22 \\
\hline $521-872$ & 38.10 & 1.50 & 37.72 & 1.485 & 102.3020 .95 & 785 & 2577 & 6 & 19 \\
\hline $521-882$ & 38.10 & 1.50 & 37.85 & 1.490 & 102.6521 .02 & 802 & 2630 & 5 & 18 \\
\hline $521-892$ & 38.10 & 1.50 & 37.85 & 1.490 & 102.6521 .02 & 796 & 2612 & 10 & 33 \\
\hline
\end{tabular}

0.50 cal. APM2, $0^{\circ}$ Obliquity Ballistic Performance

\begin{tabular}{|c|c|c|c|c|c|c|c|c|c|}
\hline \multirow[t]{2}{*}{ Plate ID } & \multicolumn{2}{|c|}{$\begin{array}{l}\text { Nominal } \\
\text { Thickness }\end{array}$} & \multicolumn{2}{|c|}{$\begin{array}{c}\text { Actual } \\
\text { Thickness }\end{array}$} & $\begin{array}{c}\text { Areal } \\
\text { Density }\end{array}$ & \multicolumn{2}{|c|}{$\begin{array}{l}\text { Ballistic } \\
\text { Limit }\end{array}$} & \multicolumn{2}{|c|}{$\begin{array}{l}\text { Standard } \\
\text { Deviation }\end{array}$} \\
\hline & $\mathbf{m m}$ & in & mm & in & $\mathrm{kg} / \mathrm{m}^{2} \quad \mathrm{psf}$ & $\mathbf{m} / \mathbf{s}$ & fps & $\mathbf{m} / \mathbf{s}$ & fps \\
\hline $521-801$ & 19.05 & 0.75 & 19.02 & 0.749 & 51.6010 .57 & 420 & 1377 & 8 & 25 \\
\hline $521-811$ & 19.05 & 0.75 & 19.05 & 0.750 & 51.6710 .58 & 418 & 1373 & 8 & 25 \\
\hline $521-901$ & 50.80 & 2.00 & 49.78 & 1.960 & 135.0327 .66 & 681 & 2234 & 8 & 26 \\
\hline $521-911$ & 50.80 & 2.00 & 50.88 & 2.003 & 137.9928 .26 & 697 & 2286 & 7 & 24 \\
\hline $521-921$ & 50.80 & 2.00 & 51.05 & 2.010 & 138.4728 .36 & 699 & 2294 & 8 & 25 \\
\hline $521-931$ & 57.15 & 2.25 & 56.90 & 2.240 & 154.3231 .61 & 738 & 2423 & 8 & 27 \\
\hline $521-941$ & 63.50 & 2.50 & 63.32 & 2.493 & 171.7435 .18 & 785 & 2574 & 8 & 26 \\
\hline $521-942$ & 63.50 & 2.50 & 63.37 & 2.495 & 171.8835 .20 & 798 & 2619 & 5 & 16 \\
\hline $521-951$ & 76.20 & 3.00 & 76.28 & 3.003 & 206.8842 .37 & 902 & 2961 & 4 & 13 \\
\hline
\end{tabular}


0.50 cal. FSP, $0^{\circ}$ Obliquity Ballistic Performance

\begin{tabular}{ccccccccccc}
\hline \multirow{2}{*}{ Plate ID } & \multicolumn{2}{c}{$\begin{array}{c}\text { Nominal } \\
\text { Thickness }\end{array}$} & \multicolumn{2}{c}{ Actual } & \multicolumn{2}{c}{$\begin{array}{c}\text { Areal } \\
\text { Thickness }\end{array}$} & \multicolumn{2}{c}{$\begin{array}{c}\text { Ballistic } \\
\text { Density }\end{array}$} & \multicolumn{2}{c}{$\begin{array}{c}\text { Simit } \\
\text { Limdard } \\
\text { Dm }\end{array}$} \\
& mm & in & mm & in & kg/m & psf & m/s & fps & m/s & fps \\
\hline $521-771$ & 22.23 & 0.88 & 22.23 & 0.875 & 60.28 & 12.35 & 765 & 2509 & 7 & 23 \\
$521-802$ & 19.05 & 0.75 & 19.15 & 0.754 & 51.94 & 10.64 & 563 & 1848 & 8 & 25 \\
$521-812$ & 19.05 & 0.75 & 19.15 & 0.754 & 51.94 & 10.64 & 573 & 1879 & 6 & 21 \\
$521-821$ & 25.40 & 1.00 & 25.60 & 1.008 & 69.44 & 14.22 & 941 & 3086 & 8 & 26 \\
$521-822$ & 25.40 & 1.00 & 25.04 & 0.986 & 67.93 & 13.91 & 911 & 2989 & 9 & 29 \\
$521-831$ & 25.40 & 1.00 & 25.40 & 1.000 & 68.89 & 14.11 & 917 & 3008 & 4 & 14 \\
$521-832$ & 25.40 & 1.00 & 25.40 & 1.000 & 68.89 & 14.11 & 922 & 3026 & 5 & 18 \\
$521-841$ & 25.40 & 1.00 & 25.53 & 1.005 & 69.24 & 14.18 & 934 & 3066 & 10 & 32 \\
$521-842$ & 25.40 & 1.00 & 25.17 & 0.991 & 68.27 & 13.98 & 904 & 2966 & 6 & 20 \\
\hline
\end{tabular}

$20 \mathrm{~mm}$ FSP, $0^{\circ}$ Obliquity Ballistic Performance

\begin{tabular}{ccccccccccc}
\hline \multirow{2}{*}{ Plate ID } & \multicolumn{2}{c}{$\begin{array}{c}\text { Nominal } \\
\text { Thickness }\end{array}$} & \multicolumn{2}{c}{$\begin{array}{c}\text { Actual } \\
\text { Thickness }\end{array}$} & \multicolumn{2}{c}{$\begin{array}{c}\text { Areal } \\
\text { Density }\end{array}$} & \multicolumn{2}{c}{$\begin{array}{c}\text { Ballistic } \\
\text { Limit }\end{array}$} & \multicolumn{2}{c}{$\begin{array}{c}\text { Standard } \\
\text { Deviation }\end{array}$} \\
& mm & in & mm & in & kg/m & psf & m/s & fps & m/s & fps \\
\hline $521-821$ & 25.40 & 1.00 & 25.60 & 1.008 & 69.44 & 14.22 & 436 & 1431 & 8 & 25 \\
$521-822$ & 25.40 & 1.00 & 25.60 & 1.008 & 69.44 & 14.22 & 439 & 1440 & 9 & 30 \\
$521-831$ & 25.40 & 1.00 & 25.40 & 1.000 & 68.89 & 14.11 & 426 & 1397 & 6 & 21 \\
$521-832$ & 25.40 & 1.00 & 25.81 & 1.016 & 69.99 & 14.34 & 431 & 1414 & 7 & 22 \\
$521-841$ & 25.40 & 1.00 & 25.53 & 1.005 & 69.24 & 14.18 & 414 & 1358 & 6 & 19 \\
$521-842$ & 25.40 & 1.00 & 25.65 & 1.010 & 69.58 & 14.25 & 407 & 1336 & 7 & 23 \\
$521-852$ & 31.75 & 1.25 & 31.50 & 1.240 & 85.42 & 17.50 & 578 & 1898 & 8 & 26 \\
$521-861$ & 31.75 & 1.25 & 31.75 & 1.250 & 86.11 & 17.64 & 574 & 1882 & 7 & 23 \\
$521-872$ & 38.10 & 1.50 & 37.72 & 1.485 & 102.3020 .95 & 799 & 2620 & 7 & 24 \\
$521-882$ & 38.10 & 1.50 & 37.85 & 1.490 & 102.6521 .02 & 787 & 2583 & 10 & 33 \\
$521-892$ & 38.10 & 1.50 & 37.85 & 1.490 & 102.6521 .02 & 758 & 2487 & 9 & 28 \\
\hline
\end{tabular}


INTENTIONALLY LEFT BLANK. 
List of Symbols, Abbreviations, and Acronyms

$\begin{array}{ll}\text { AA } & \text { aluminum alloy } \\ \text { AP } & \text { armor-piercing } \\ \text { ARL } & \text { US Army Research Laboratory } \\ \text { ATC } & \text { Aberdeen Test Center } \\ \text { CP } & \text { complete penetration } \\ \text { DAC } & \text { Defense Acquisition Challenge } \\ \text { EF } & \text { experimental facility } \\ \text { FSP } & \text { fragment-simulating projectile } \\ \text { IR } & \text { infrared } \\ \text { OSD } & \text { Office of the Secretary of Defense } \\ \text { PP } & \text { partial penetration }\end{array}$




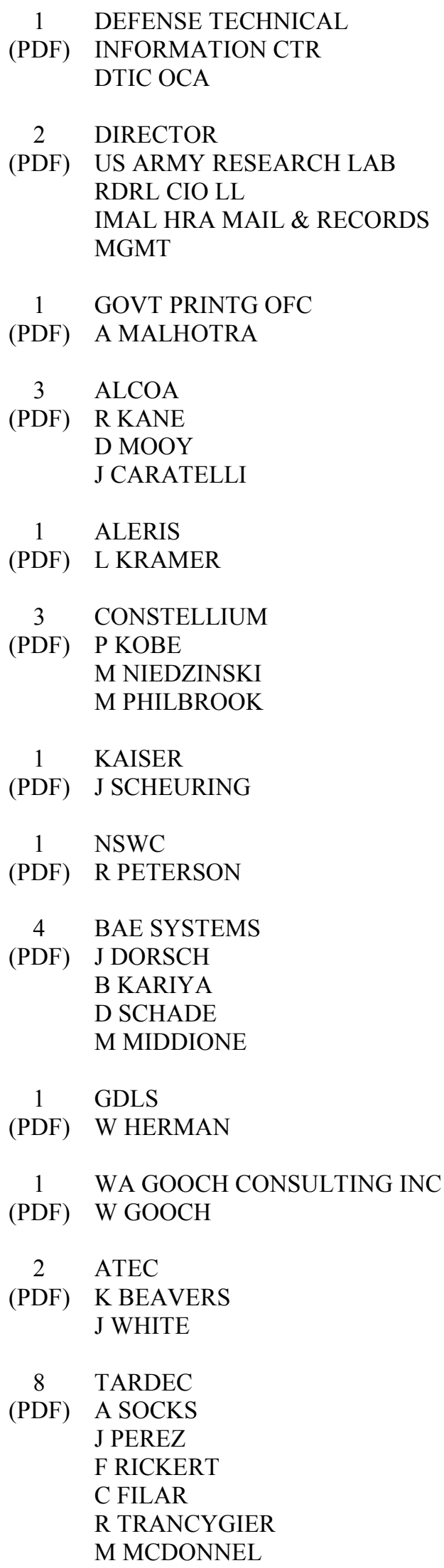

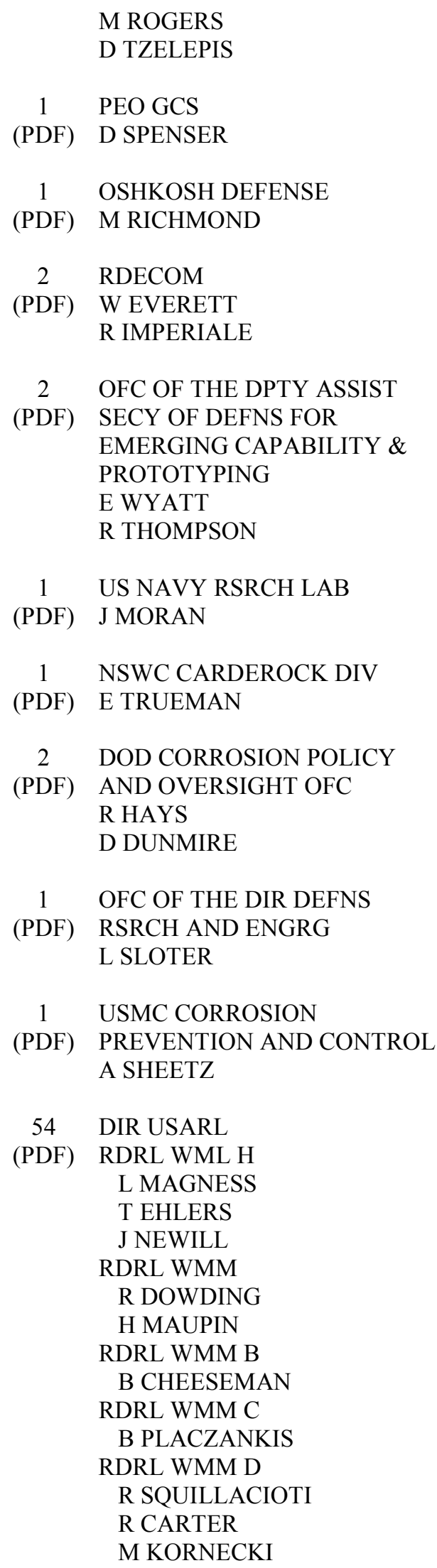




RDRL WMM F
K DOHERTY
J CHINELLA
E KLIER
S GRENDAHL
RDRL WMP D
J RUNYEON
D KLEPONIS
D PETTY
M KEELE
RDRL WMP E
P BARTKOWSKI
S BARTUS
W BRYANT
M BURKINS
D CHURN
P DAVIS
B DAWSON
A DUCOTE
K DUDECK
D GALLARDY
W GOOCH
D HACKBARTH
D HANDSHOE
J HOGAN
D HORNBAKER
E HORWATH
J HOUSKAMP
T JONES
M KLUSEWITZ
C KRAUTHAUSER
J LE
D LITTLE
K LLEWELLYN
K MCNAB
J MONTGOMERY
T NELLENBACH
T O'CONNOR
P PEREGINO
T QUIGG
B SANDERSON
D SCHALL
D SHOWALTER
N STURGILL
P SWOBODA
C WALTER
RDRL WMP F
N GNIAZDOWSKI


INTENTIONALLY LEFT BLANK. 